Article

\title{
Efficient Syntheses of 1,2,3-Triazoloamide Derivatives Using Solid- and Solution-Phase Synthetic Approaches
}

\author{
Doohyun Lee ${ }^{1}$, Daehun Kim ${ }^{1}$, Seungyeon Lee ${ }^{1}$, Taegeum Kim ${ }^{1}$, Joobin Kim ${ }^{1}$, Sohee Kim ${ }^{1}$, \\ Kwang-Hyeon Liu ${ }^{1}$, Sangkyu Lee ${ }^{1}$, Jong-Sup Bae ${ }^{1}$, Kyung-Sik Song ${ }^{1}$, Chang-Woo Cho ${ }^{2}$, \\ Youn Kyung Son ${ }^{3}$, Dong Jae Baek ${ }^{4, *}$ and Taeho Lee ${ }^{1, *}$
}

Received: 16 September 2015 ; Accepted: 29 October 2015 ; Published: 5 November 2015

Academic Editors: Wim Dehaen and Derek J. McPhee

1 College of Pharmacy, Research Institute of Pharmaceutical Sciences, Kyungpook National University, 80 Daehak-ro, Buk-gu, Daegu 702-701, Korea; newkiy@hanmail.net (D.L.); eogns1201@nate.com (D.K.); tmddusj@naver.com (S.L.); eksvnddlv321@naver.com (T.K.); joobin87@naver.com (J.K.); ksh71051@naver.com (S.K.); dstlkh@knu.ac.kr (K.-H.L.); sangkyu@knu.ac.kr (S.L.); baejs@knu.ac.kr (J.-S.B.); kssong@knu.ac.kr (K.-S.S.)

2 Department of Chemistry, Kyungpook National University, 80 Daehak-ro, Buk-gu, Daegu 702-701, Korea; cwcho@knu.ac.kr

3 National Institute of Biological Resources, Hwangyeong-ro 42, Seo-gu, Incheon 404-708, Korea; sophy004@korea.kr

4 College of Pharmacy, Natural Medicine Research Institute, Mokpo National University, 1666 Youngsan-ro, Muan-gun, Jeonnam 534-729, Korea

* Correspondence: dbaek@mokpo.ac.kr (D.J.B.); tlee@knu.ac.kr (T.L.); Tel.: +82-53-950-8573 (T.L.); Fax: +82-53-950-8557 (T.L.)

\begin{abstract}
Efficient synthetic routes for the preparation of secondary and tertiary 1,2,3-triazoloamide derivatives were developed. A secondary $\alpha-1,2,3$-triazoloamide library was constructed and expanded by a previously developed solid-phase synthetic route and a tertiary 1,2,3-triazoloamide library was constructed by a parallel solution-phase synthetic route. The synthetic routes rely on amide formation with secondary amines and chloro-acid chlorides; $\mathrm{S}_{\mathrm{N}} 2$ reaction with sodium azide; and the selective [3 +2$]$ Hüisgen cycloaddition with appropriate terminal alkynes. The target secondary and tertiary 1,2,3-triazoloamide derivatives were obtained with three-diversity points in excellent overall yields and purities using the reported solid- and solution-phase synthetic routes, respectively.
\end{abstract}

Keywords: 1,2,3-triazoloamide; solid-phase synthesis; solution-phase synthesis

\section{Introduction}

Combinatorial chemistry has emerged as a powerful technique for the synthesis of biologically active small molecules for the purpose of medicinal chemistry programs within the pharmaceutical industry [1-5]. Recently, the 1,2,3-triazole moiety, produced by $\mathrm{Cu}(\mathrm{I})$-catalyzed [3 +2 ] cycloaddition reactions, has been used as a scaffold for generating combinatorial libraries [6-10]. 1,2,3-Triazoles can mimic the topological and electronic features of an amide bond, and this be used as bioisosteres of the amide moiety. They are particularly stable to reduction, oxidation, and hydrolysis conditions.

Various $\alpha-1,2,3$-triazoloamide derivatives have been shown to exhibit a wide range of biological activities [11-19]. In recent examples, $\alpha-1,2,3$-triazoloamide related compounds have been developed and studied as tropomysin receptor kinase A (TrkA) inhibitors [11], as inhibitors of Mycobacterium tuberculosis [12], as phosphodiesterase 4B (PDE4B) inhibitor for anticancer agents [13], as quorum 
sensing modulators [14], as $\beta$-haematin inhibitors for antimalarial agents [15], as $\gamma$-secretase modulators [16], as protein tyrosine phosphatase (PTPs) inhibitors [17], as lymphoid tyrosin phosphatase (Lyp, PTPN22) inhibitors [18], and as glucokinase (GK) acitvators [19].

Previously, we have reported a solid-phase synthetic protocol for the preparation of secondary $\alpha-1,2,3$-triazoloamides $1\left(R^{2}=H\right.$, Figure 1) [20]. However, an expanded $\alpha-1,2,3$-triazoloamide library was needed for our drug discovery project, which includes the secondary and tertiary 1,2,3-triazoloamides. Herein, we describe the construction of expanded libraries of secondary $\alpha-1,2,3$-triazoloamides $\mathbf{1}$ on solid-phase and of tertiary 1,2,3-triazoloamides 2 in parallel solution-phase, which is applicable to high-throughput construction of drug-like compound libraries.

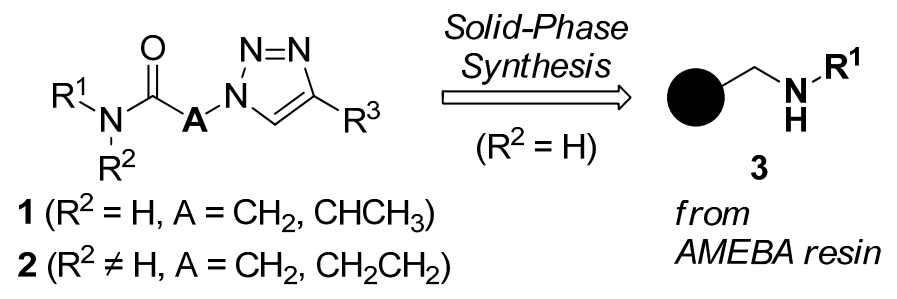

Figure 1. Structure and use of 1,2,3-triazoloamides 1 and 2.

\section{Results and Discussion}

The synthetic sequence for secondary $\alpha-1,2,3$-triazoloamides $\mathbf{1}\left(\mathrm{R}^{2}=H\right)$ is shown in Scheme 1 [20]. According to the solid-phase synthetic approach with the polymer-bound amines 3 , which were prepared by reductive amination reaction from Acid sensitive Methoxy Benzaldehyde (AMEBA) [20,21] resin 4 and primary amines 5 (the first diversity element $R^{1}$; Figure 2), polymer-bound chloroamides 7 can be easily prepared by the reaction of amine resin 3 with chloro-acid chloride 6 (the second diversity element $\mathbf{A}$; Figure 3) and triethylamine in $\mathrm{CH}_{2} \mathrm{Cl}_{2}$ at room temperature. Treatment of solid supported chloroamides $7\left(\mathrm{R}=\mathrm{Cl}, \mathrm{A}=\mathrm{CH}_{2}\right.$ or $\left.\mathrm{CHCH}_{3}\right)$ with sodium azide in DMF at room temperature, provides the $\alpha$-azidoamide resin $8\left(R=N_{3}\right)$.

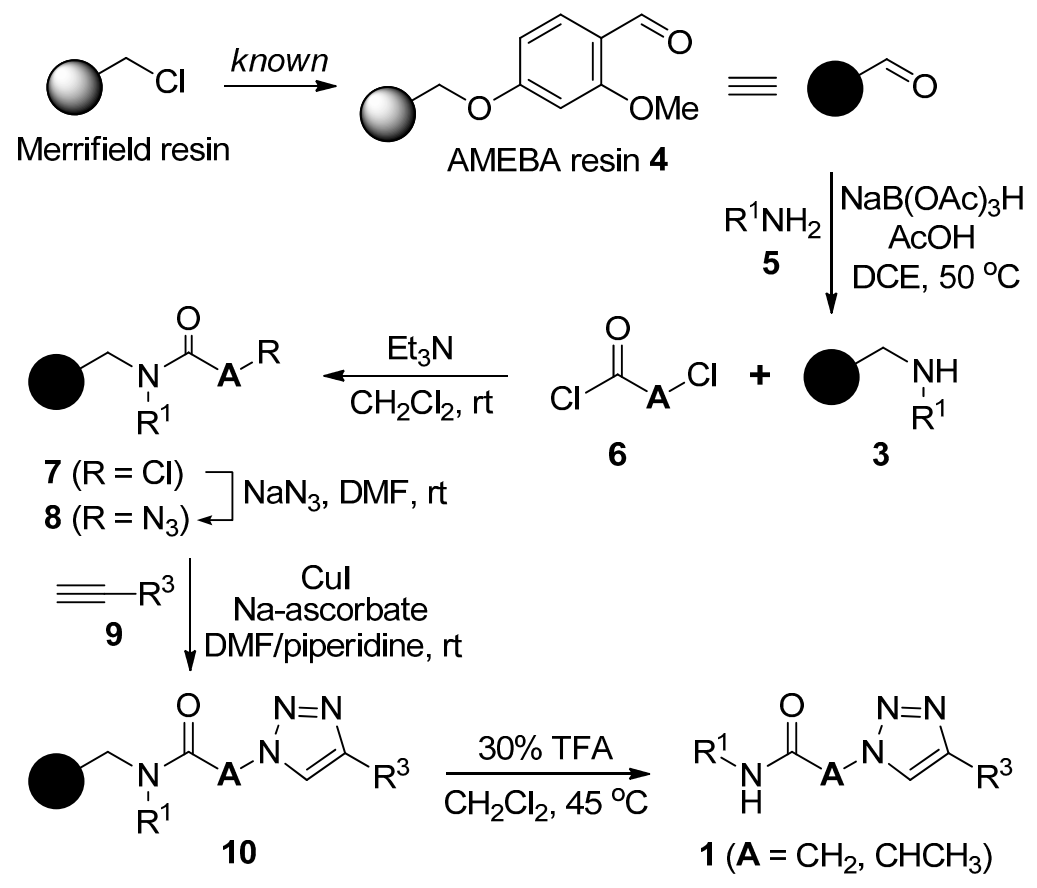

Scheme 1. Solid-phase synthesis of secondary $\alpha-1,2,3$-triazoloamide derivatives 1 . 
<smiles>Nc1ccccc1</smiles>
$5 \mathbf{a}$<smiles>COc1ccc(N)cc1</smiles>
$5 b$
$5 c$<smiles>COc1ccccc1N</smiles>

5d<smiles>CCCCN</smiles>

$5 e$

Figure 2. Diversity reagents 5 for secondary $\alpha-1,2,3$-triazoloamides $\mathbf{1}$.<smiles>O=C(Cl)CCl</smiles>

$6 a$<smiles>CC(Cl)C(=O)Cl</smiles>

$6 b$<smiles>O=C(Cl)CCCl</smiles>

$6 c$

Figure 3. Diversity reagents 6 for 1,2,3-triazoloamides 1 and 2.

In the case of $\beta$-chloroamide 7ac, which was prepared by the reaction of amine resin $3 \mathbf{a}$ and 3-chloropropionyl chloride $(\mathbf{6 c})$, the $\mathrm{S}_{\mathrm{N}} 2$ reaction with sodium azide gave the undesired acrylamide 12 because of an elimination of $\beta$-chloroamide (Scheme 2). The reaction was confirmed by ATR-FTIR analysis of resin $\mathbf{1 1}$ and the cleavage of the resin $\mathbf{1 1}$ under 30\% TFA in $\mathrm{CH}_{2} \mathrm{Cl}_{2}$ at room temperature provided an $N$-phenylacrylamide (12) $[22,23]$ as a major product.

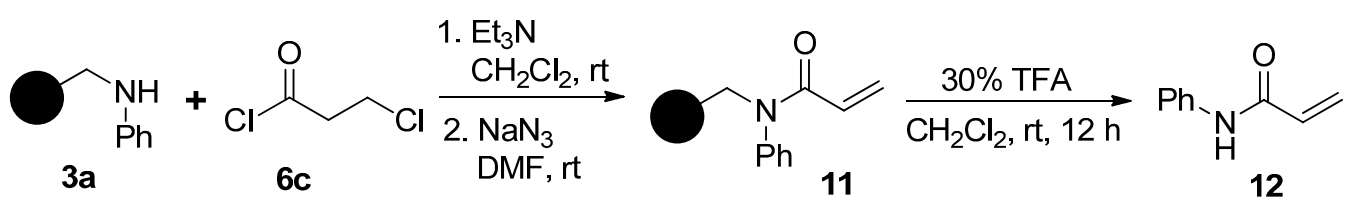

Scheme 2. Reaction of amine resin 3a and 3-chloropropionyl chloride (6c).

The selective [3 + 2] Hüisgen cycloaddition [24-29] was performed with $\alpha$-azidoamide resin 8 and terminal acetylene 9 (the third diversity element $R^{3}$; Figure 4 ) according to optimized reaction condition (3 equiv. CuI, 3 equiv. sodium ascorbate, DMF/piperidine (4:1), room temperature) [20]. The well-known methods for the synthesis of 1,2,3-triazoles (catalytic $\mathrm{CuSO}_{4}$ /sodium ascorbate or $\mathrm{CuI} /$ diisopropylethylamine as reagents and $\mathrm{H}_{2} \mathrm{O} / \mathrm{t}-\mathrm{BuOH}, \mathrm{EtOH}$, or THF as solvent) were not very efficient. Under the general cleavage conditions of AMEBA resin $\left(30 \% \mathrm{TFA}, \mathrm{CH}_{2} \mathrm{Cl}_{2}\right.$, room temperature), the resulting polymer-bound product 10aaa gave the desired $\alpha-1,2,3$-triazoloamide 1aaa $(44 \%)$ and by-product $13(30 \%)$, while unreacted resin 10aaa remained as was confirmed by ATR-FTIR analysis (Scheme 3).<smiles>C#Cc1ccccc1</smiles>

9a<smiles>C#Cc1ccc(OC)cc1</smiles><smiles>C#Cc1ccc(C#N)cc1</smiles><smiles>C#Cc1ccsc1</smiles><smiles>C#Cc1ccccn1</smiles><smiles>C#CCCCC</smiles><smiles>C#Cc1cccc(OC)c1</smiles>

9g<smiles>C#Cc1ccccc1OC</smiles><smiles>[HgH2]</smiles><smiles>C#Cc1ccc(C)cc1</smiles>

9i<smiles>C#Cc1ccc(F)cc1F</smiles>

9n<smiles>C#Cc1cccc(C)c1</smiles>

9j<smiles>C#CCc1ccccc1</smiles><smiles>C#Cc1ccccc1C</smiles>

9k<smiles>C#Cc1ccc(N(C)C)cc1</smiles>

Figure 4. Diversity reagents 9 for 1,2,3-triazoloamides 1 and 2. 


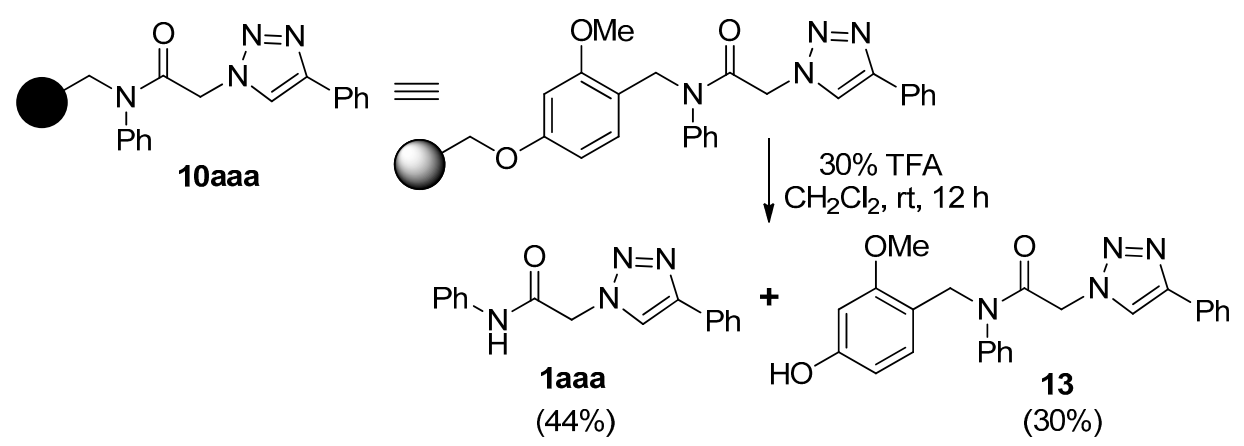

Scheme 3. Cleavage of $\alpha-1,2,3$-triazoloamide resin 10aaa.

Finally, the $\alpha-1,2,3$-triazoloamide resin 10aaa was cleaved from the solid support under 30\% TFA in $\mathrm{CH}_{2} \mathrm{Cl}_{2}$ at $45^{\circ} \mathrm{C}$ to provide the desired $\alpha-1,2,3$-triazoloamide 1aaa $[24,27,30]$ (93\% over six steps, from Merrifield resin) without formation of by-product 13.

The reaction progress on solid-phase was monitored by ATR-FTIR (Figure 5). The progress of reductive amination of AMEBA resin 4 and amine $5 a\left(R^{1}=P h\right)$ was checked by the appearance of the weak NH stretching band at $3424 \mathrm{~cm}^{-1}$ and the disappearance of the aldehyde stretching band at $1678 \mathrm{~cm}^{-1}$. The progression of amide formation for $7 \mathrm{aa}\left(\mathrm{R}^{1}=\mathrm{Ph}, \mathbf{A}=\mathrm{CH}_{2}\right)$ was monitored by ATR-FTIR which displayed the disappearance of the characteristic NH band at $3424 \mathrm{~cm}^{-1}$ and appearance of the amide carbonyl stretching band at $1666 \mathrm{~cm}^{-1}$. The $\mathrm{S}_{\mathrm{N}} 2$ reaction of $7 \mathrm{aa}\left(\mathrm{R}^{1}=\mathrm{Ph}\right.$, $\mathbf{A}=\mathrm{CH}_{2}$ ) with sodium azide was monitored by the appearance of the azide stretching band at $2101 \mathrm{~cm}^{-1}$. The completion of selective [3 +2] Hüisgen cycloaddition of $7 \mathbf{a a}$ and $\mathbf{9 a}$ was confirmed by the disappearance of the azide stretching band.

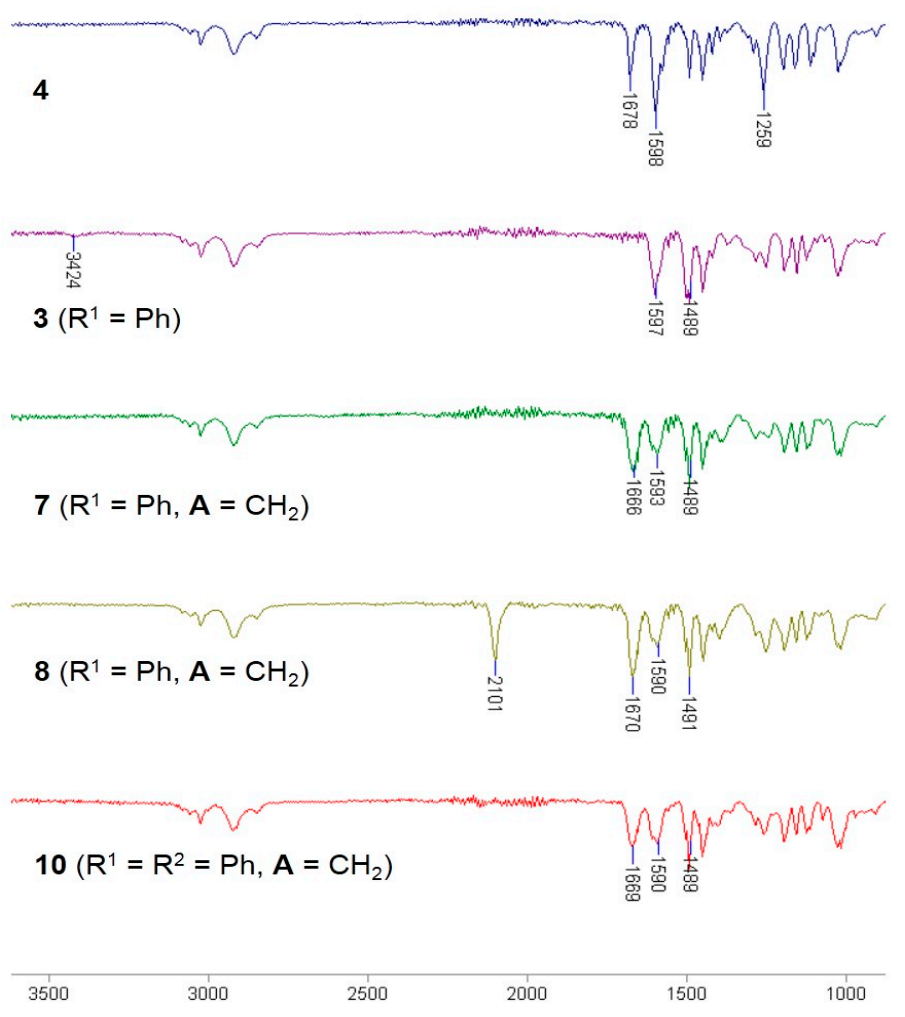

Figure 5. ATR-FTIR spectra of resins 3, 4, 7, 8 and $10\left(\mathrm{R}^{1}=\mathrm{R}^{2}=\mathrm{Ph}, \mathrm{A}=\mathrm{CH}_{2}\right)$. 
Following the optimized solid-phase synthetic route, the secondary $\alpha-1,2,3$-triazoloamide derivatives 1 were prepared starting from Merrifield resin and appropriate primary amines 5 $\left(\mathrm{R}^{1} \mathrm{NH}_{2}\right.$; Figure 2), $\alpha$-chloroacetyl chlorides $\mathbf{6 a}$ and $\mathbf{6 b}(\mathrm{Cl}-\mathbf{A}-\mathrm{COCl}$; Figure 3), and terminal acetylenes $9\left(\mathrm{R}^{3} \mathrm{C} \equiv \mathrm{CH}\right.$; Figure 4$)$ and the products displayed in Table 1 . In most cases, secondary $\alpha-1,2,3$-triazoloamide derivatives 1 (80 examples) were obtained with high yields (94\%-75\%) and high purities, $>95 \%$ as judged from LC-MS traces (integration of 200-400 nm diode array traces).

Table 1. Prepared secondary $\alpha-1,2,3$-triazoloamide derivatives $\mathbf{1}^{\mathrm{a}}$.<smiles>[R1]NC(=O)[Y]n1cc([R])nn1</smiles>

\begin{tabular}{|c|c|c|c|c|c|c|c|c|c|c|c|}
\hline Entry & Product & ts $R^{1}$ & $\mathbf{A}$ & $\mathbf{R}^{3}$ & $\begin{array}{l}\text { Yield } \\
(\%)^{b}\end{array}$ & Entry & Products & $\mathbf{R}^{1}$ & $\mathbf{A}$ & $\mathbf{R}^{3}$ & $\begin{array}{l}\text { Yield } \\
(\%)^{b}\end{array}$ \\
\hline 1 & 1aaa & $\mathrm{Ph}$ & $\mathrm{CH}_{2}$ & $\mathrm{Ph}$ & 93 & 41 & 1dan & 2-MeO-Ph & $\mathrm{CH}_{2}$ & 2,4-di-F-Ph & 94 \\
\hline 2 & 1aab & $\mathrm{Ph}$ & $\mathrm{CH}_{2}$ & 4-MeO-Ph & 91 & 42 & 1dao & 2-MeO-Ph & $\mathrm{CH}_{2}$ & $\mathrm{Bn}$ & 88 \\
\hline 3 & 1aac & $\mathrm{Ph}$ & $\mathrm{CH}_{2}$ & 4-CN-Ph & 83 & 43 & 1eaa & $n$-Bu & $\mathrm{CH}_{2}$ & $\mathrm{Ph}$ & 81 \\
\hline 4 & 1aad & $\mathrm{Ph}$ & $\mathrm{CH}_{2}$ & 3-thiophenyl & 82 & 44 & 1eab & $n-\mathrm{Bu}$ & $\mathrm{CH}_{2}$ & 4-MeO-Ph & 88 \\
\hline 5 & 1aae & $\mathrm{Ph}$ & $\mathrm{CH}_{2}$ & 2-pyridyl & 85 & 45 & 1eac & $n-\mathrm{Bu}$ & $\mathrm{CH}_{2}$ & 4-CN-Ph & 83 \\
\hline 6 & 1aaf & $\mathrm{Ph}$ & $\mathrm{CH}_{2}$ & $n-\mathrm{Bu}$ & 92 & 46 & 1ead & $n-\mathrm{Bu}$ & $\mathrm{CH}_{2}$ & 3-thiophenyl & 80 \\
\hline 7 & 1baa 4 & 4-MeO-Ph & $\mathrm{CH}_{2}$ & $\mathrm{Ph}$ & 92 & 47 & 1eae & $n$-Bu & $\mathrm{CH}_{2}$ & 2-pyridyl & 89 \\
\hline 8 & 1bab 4 & 4-MeO-Ph & $\mathrm{CH}_{2}$ & 4-MeO-Ph & 94 & 48 & 1eaf & $n$-Bu & $\mathrm{CH}_{2}$ & $n-\mathrm{Bu}$ & 89 \\
\hline 9 & 1bac 4 & 4-MeO-Ph & $\mathrm{CH}_{2}$ & 4-CN-Ph & 89 & 49 & 1faa & $i-\operatorname{Pr}$ & $\mathrm{CH}_{2}$ & $\mathrm{Ph}$ & 79 \\
\hline 10 & 1bad 4 & 4-MeO-Ph & $\mathrm{CH}_{2}$ & 3-thiophenyl & 85 & 50 & $1 \mathrm{fab}$ & $i-\operatorname{Pr}$ & $\mathrm{CH}_{2}$ & 4-MeO-Ph & 87 \\
\hline 11 & 1bag 4 & 4-MeO-Ph & $\mathrm{CH}_{2}$ & 3-MeO-Ph & 87 & 51 & 1fac & $i-\operatorname{Pr}$ & $\mathrm{CH}_{2}$ & 4-CN-Ph & 75 \\
\hline 12 & 1baj 4 & 4-MeO-Ph & $\mathrm{CH}_{2}$ & 3-Me-Ph & 89 & 52 & $1 \mathrm{fad}$ & $i-\operatorname{Pr}$ & $\mathrm{CH}_{2}$ & 3-thiophenyl & 77 \\
\hline 13 & 1bak 4 & 4-MeO-Ph & $\mathrm{CH}_{2}$ & 2-Me-Ph & 84 & 53 & 1fae & $i-\operatorname{Pr}$ & $\mathrm{CH}_{2}$ & 2-pyridyl & 84 \\
\hline 14 & 1bal 4 & 4-MeO-Ph & $\mathrm{CH}_{2}$ & $4-\mathrm{NMe}_{2}-\mathrm{Ph}$ & 79 & 54 & 1faf & $i-\operatorname{Pr}$ & $\mathrm{CH}_{2}$ & $n-\mathrm{Bu}$ & 83 \\
\hline 15 & 1bam 4 & 4-MeO-Ph & $\mathrm{CH}_{2}$ & 4-PhO-Ph & 92 & 55 & $1 \mathrm{dba}$ & 2-MeO-Ph & $\mathrm{CHCH}_{3}$ & $\mathrm{Ph}$ & 88 \\
\hline 16 & 1ban 4 & 4-MeO-Ph & $\mathrm{CH}_{2}$ & 2,4-di-F-Ph & 87 & 56 & $1 \mathrm{dbb}$ & 2-MeO-Ph & $\mathrm{CHCH}_{3}$ & 4-MeO-Ph & 91 \\
\hline 17 & 1bao 4 & 4-MeO-Ph & $\mathrm{CH}_{2}$ & $\mathrm{Bn}$ & 85 & 57 & $1 \mathrm{dbc}$ & 2-MeO-Ph & $\mathrm{CHCH}_{3}$ & 4-CN-Ph & 87 \\
\hline 18 & 1caa 3 & 3-MeO-Ph & $\mathrm{CH}_{2}$ & $\mathrm{Ph}$ & 91 & 58 & $1 \mathrm{dbd}$ & 2-MeO-Ph & $\mathrm{CHCH}_{3}$ & 3-thiophenyl & 80 \\
\hline 19 & 1cab 3 & 3-MeO-Ph & $\mathrm{CH}_{2}$ & 4-MeO-Ph & 90 & 59 & 1dbe & 2-MeO-Ph & $\mathrm{CHCH}_{3}$ & 2-pyridyl & 83 \\
\hline 20 & 1cac & 3-MeO-Ph & $\mathrm{CH}_{2}$ & 4-CN-Ph & 87 & 60 & $1 \mathrm{dbg}$ & 2-MeO-Ph & $\mathrm{CHCH}_{3}$ & 3-MeO-Ph & 94 \\
\hline 21 & 1cad 3 & 3-MeO-Ph & $\mathrm{CH}_{2}$ & 3-thiophenyl & 76 & 61 & $1 \mathrm{dbh}$ & 2-MeO-Ph & $\mathrm{CHCH}_{3}$ & 2-MeO-Ph & 89 \\
\hline 22 & 1cae 3 & 3-MeO-Ph & $\mathrm{CH}_{2}$ & 2-pyridyl & 81 & 62 & $1 \mathrm{dbi}$ & 2-MeO-Ph & $\mathrm{CHCH}_{3}$ & 4-Me-Ph & 94 \\
\hline 23 & 1cah 3 & 3-MeO-Ph & $\mathrm{CH}_{2}$ & 2-MeO-Ph & 83 & 63 & $1 \mathrm{dbj}$ & 2-MeO-Ph & $\mathrm{CHCH}_{3}$ & 3-Me-Ph & 83 \\
\hline 24 & 1caj 3 & 3-MeO-Ph & $\mathrm{CH}_{2}$ & 3-Me-Ph & 93 & 64 & $1 \mathrm{dbk}$ & 2-MeO-Ph & $\mathrm{CHCH}_{3}$ & 2-Me-Ph & 74 \\
\hline 25 & 1cak 3 & 3-MeO-Ph & $\mathrm{CH}_{2}$ & 2-Me-Ph & 90 & 65 & $1 \mathrm{dbl}$ & 2-MeO-Ph & $\mathrm{CHCH}_{3}$ & $4-\mathrm{NMe}_{2}-\mathrm{Ph}$ & 82 \\
\hline 26 & $1 \mathrm{cal}$ & 3-MeO-Ph & $\mathrm{CH}_{2}$ & $4-\mathrm{NMe}_{2}-\mathrm{Ph}$ & 91 & 66 & $1 \mathrm{dbm}$ & 2-MeO-Ph & $\mathrm{CHCH}_{3}$ & 4-PhO-Ph & 87 \\
\hline 27 & 1cam 3 & 3-MeO-Ph & $\mathrm{CH}_{2}$ & 4-PhO-Ph & 91 & 67 & $1 \mathrm{dbn}$ & 2-MeO-Ph & $\mathrm{CHCH}_{3}$ & 2,4-di-F-Ph & 92 \\
\hline 28 & 1can 3 & 3-MeO-Ph & $\mathrm{CH}_{2}$ & 2,4-di-F-Ph & 92 & 68 & $1 \mathrm{dbo}$ & 2-MeO-Ph & $\mathrm{CHCH}_{3}$ & $\mathrm{Bn}$ & 86 \\
\hline 29 & 1cao 3 & 3-MeO-Ph & $\mathrm{CH}_{2}$ & Bn & 88 & 69 & 1eba & $n-\mathrm{Bu}$ & $\mathrm{CHCH}_{3}$ & $\mathrm{Ph}$ & 83 \\
\hline 30 & 1daa 2 & 2-MeO-Ph & $\mathrm{CH}_{2}$ & $\mathrm{Ph}$ & 94 & 70 & $1 e b b$ & $n-\mathrm{Bu}$ & $\mathrm{CHCH}_{3}$ & 4-MeO-Ph & 81 \\
\hline 31 & 1dab 2 & 2-MeO-Ph & $\mathrm{CH}_{2}$ & 4-MeO-Ph & 90 & 71 & $1 e b c$ & $n-\mathrm{Bu}$ & $\mathrm{CHCH}_{3}$ & 4-CN-Ph & 79 \\
\hline 32 & 1dac 2 & 2-MeO-Ph & $\mathrm{CH}_{2}$ & 4-CN-Ph & 86 & 72 & 1ebd & $n$-Bu & $\mathrm{CHCH}_{3}$ & 3-thiophenyl & 78 \\
\hline 33 & 1dad 2 & 2-MeO-Ph & $\mathrm{CH}_{2}$ & 3-thiophenyl & 91 & 73 & 1ebe & $n-\mathrm{Bu}$ & $\mathrm{CHCH}_{3}$ & 2-pyridyl & 84 \\
\hline 34 & 1dae 2 & 2-MeO-Ph & $\mathrm{CH}_{2}$ & 2-pyridyl & 84 & 74 & 1ebf & $n$-Bu & $\mathrm{CHCH}_{3}$ & $n-\mathrm{Bu}$ & 81 \\
\hline 35 & 1dag 2 & 2-MeO-Ph & $\mathrm{CH}_{2}$ & 3-MeO-Ph & 86 & 75 & $1 \mathrm{fba}$ & $i-\operatorname{Pr}$ & $\mathrm{CHCH}_{3}$ & $\mathrm{Ph}$ & 88 \\
\hline 36 & 1dah 2 & 2-MeO-Ph & $\mathrm{CH}_{2}$ & 2-MeO-Ph & 80 & 76 & $1 \mathrm{fbb}$ & $i-\operatorname{Pr}$ & $\mathrm{CHCH}_{3}$ & 4-MeO-Ph & 83 \\
\hline 37 & 1dai 2 & 2-MeO-Ph & $\mathrm{CH}_{2}$ & 4-Me-Ph & 78 & 77 & $1 \mathrm{fbc}$ & $i-\operatorname{Pr}$ & $\mathrm{CHCH}_{3}$ & 4-CN-Ph & 78 \\
\hline 38 & 1daj & 2-MeO-Ph & $\mathrm{CH}_{2}$ & 3-Me-Ph & 85 & 78 & $1 \mathrm{fbd}$ & $i-\operatorname{Pr}$ & $\mathrm{CHCH}_{3}$ & 3-thiophenyl & 75 \\
\hline 39 & 1dal 2 & 2-MeO-Ph & $\mathrm{CH}_{2}$ & $4-\mathrm{NMe}_{2}-\mathrm{Ph}$ & 88 & 79 & 1fbe & $i-\operatorname{Pr}$ & $\mathrm{CHCH}_{3}$ & 2-pyridyl & 81 \\
\hline 40 & 1dam 2 & 2-MeO-Ph & $\mathrm{CH}_{2}$ & 4-PhO-Ph & 92 & 80 & $1 \mathrm{fbf}$ & $i-\operatorname{Pr}$ & $\mathrm{CHCH}_{3}$ & $n$-Bu & 82 \\
\hline
\end{tabular}

${ }^{a}$ All reactions were performed on 150-200 mg scale of resin $\mathbf{1 0}$ and the purities of compounds $\mathbf{1}$ were over $95 \%$ as judged from LC-MS traces (integration of diode array $200-400 \mathrm{~nm}$ traces); ${ }^{\mathrm{b}}$ Six-step overall yield from Merrifield resin (loading capacity $=0.94 \mathrm{mmol} / \mathrm{g}$ ).

With a successful synthetic route for secondary $\alpha-1,2,3$-triazoloamides 1 , the stage progressed to the tertiary 1,2,3-triazoloamides $2\left(\mathrm{R}^{2} \neq \mathrm{H}\right)$ (Scheme 4). The chloroamides 15 [31-37] were prepared from the reaction of secondary amines $\mathbf{1 4}$ (the first diversity elements $R^{1}$ and $R^{2}$; Figure 6) and chloro-acid chlorides $\mathbf{6 a}$ and $\mathbf{6 c}$ (the second diversity element A; see Figure 3) with triethylamine 
in $\mathrm{CH}_{2} \mathrm{Cl}_{2}$ at room temperature (99\%-92\% yields). Followed by $\mathrm{S}_{\mathrm{N}} 2$ reaction of tertiary amides 15 with sodium azide to generated the corresponding azidoamides $\mathbf{1 6}$ [15,17,38-41] in high yields (99\%-94\% yields) (Figure 7). In contrast to the solid-phase synthesis of secondary 1,2,3-triazoloamides 1 , treatment of tertiary $\beta$-chloroamide 15ac with sodium azide in DMF at room temperature, provided the corresponding $\beta$-azidoamide 16ac in high yield (94\% yield) without formation of the undesired acrylamide. Under the general conditions [6-10,24-28] of the Cu-catalyzed 1,3-dipolar cycloaddition (catalytic $\mathrm{CuSO}_{4}$ /sodium ascorbate) of azidoamide 16aa and terminal acetylene $9 \mathrm{a}$ in $\mathrm{H}_{2} \mathrm{O} / t-\mathrm{BuOH}$, the desired tertiary 1,2,3-triazoloamide 2aaa is generated in high yield (96\%).

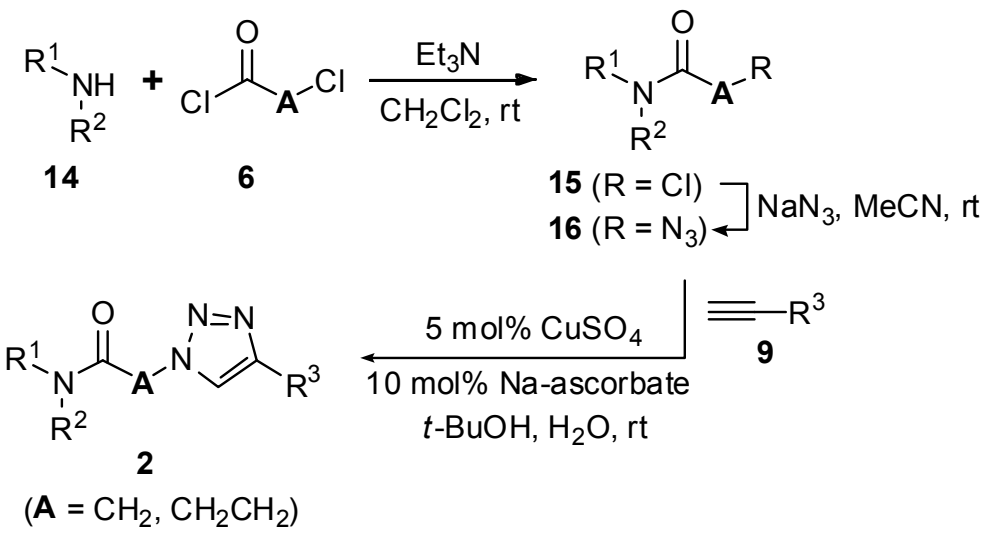

Scheme 4. Solution-phase synthesis of tertiary 1,2,3-triazoloamide derivatives 2.<smiles>C1COCCN1</smiles>

$14 a$<smiles>C1CCNCC1</smiles>

14b<smiles>C1CCNC1</smiles>

14c<smiles>C1CCCNCC1</smiles>

14d<smiles>CNc1ccccc1</smiles>

$14 \mathrm{e}$

Figure 6. Diversity reagents 14 for tertiary 1,2,3-triazoloamides 2.<smiles>[R]CC(=O)N1CCOCC1</smiles>

15aa $(\mathrm{R}=\mathrm{Cl} ; 96 \%)$

16aa $\left(R=N_{3} ; 99 \%\right)$<smiles>[R]CC(=O)N1CCCCCC1</smiles>

15da $(\mathrm{R}=\mathrm{Cl}$; 95\%)

16da ( $\left.R=N_{3} ; 96 \%\right)$<smiles>[R]CC(=O)N1CCCCC1</smiles>

15ba $(\mathrm{R}=\mathrm{Cl}$; 98\%)

16ba $\left(R=N_{3} ; 96 \%\right)$<smiles>[R]CC(=O)N(C)c1ccccc1</smiles>

15ea $(\mathrm{R}=\mathrm{Cl}$; $99 \%)$

16ea $\left(R=N_{3} ; 97 \%\right)$<smiles>[R]CC(=O)N1CCCC1</smiles>

$15 \mathrm{ca}(\mathrm{R}=\mathrm{Cl} ; 92 \%)$

16ca $\left(R=N_{3} ; 94 \%\right)$<smiles>[R]CCC(=O)N1CCOCC1</smiles>

15ac $(\mathrm{R}=\mathrm{Cl}$; 95\%)

16ac $\left(R=N_{3} ; 94 \%\right)$

Figure 7. Prepared tertiary amides $\mathbf{1 5}$ and sodium azidoamide $\mathbf{1 6 .}$

By using the parallel solution-phase synthetic route, we were able to prepare a number of tertiary 1,2,3-triazoloamide derivatives 2 displayed in Table 2 starting from appropriate secondary amines 14 $\left(\mathrm{R}^{1} \mathrm{R}^{2} \mathrm{NH}\right.$; Figure 6), chloro-acid chlorides $6 \mathbf{a}$ and $\mathbf{6 c}(\mathrm{Cl}-\mathrm{A}-\mathrm{COCl}$; Figure 3$)$, and terminal acetylenes $9\left(\mathrm{R}^{3} \mathrm{C} \equiv \mathrm{CH}\right.$; Figure 4). In most cases, tertiary 1,2,3-triazoloamide derivatives $1 \mathrm{~T}$ (80 examples) were obtained with high yields (99\%-84\%) from azidoamide 16 and in high purities, $>95 \%$ as judged from LC-MS traces (integration of 200-400 $\mathrm{nm}$ diode array traces). 
Table 2. Prepared tertiary 1,2,3-triazoloamide derivatives $2 .{ }^{\text {a }}$

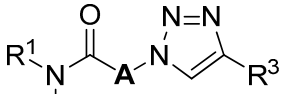

$$
\begin{aligned}
& \mathrm{R}^{2} 2
\end{aligned}
$$

\begin{tabular}{|c|c|c|c|c|c|c|c|c|c|c|c|}
\hline Entry & Products & $\mathbf{N R}^{1} \mathbf{R}^{2}$ & $\mathbf{A}$ & $\mathbf{R}^{3}$ & $\begin{array}{l}\text { Yield } \\
(\%)^{b}\end{array}$ & Entry & Products & $\mathbf{N R}^{1} \mathbf{R}^{2}$ & $\mathbf{A}$ & $\mathbf{R}^{3}$ & $\begin{array}{l}\text { Yield } \\
(\%)^{b}\end{array}$ \\
\hline 1 & 2aaa & morpholine & $\mathrm{CH}_{2}$ & $\mathrm{Ph}$ & 91 & 41 & 2 can & pyrrolidine & $\mathrm{CH}_{2}$ & 2,4-di-F-Ph & 86 \\
\hline 2 & $2 a a b$ & morpholine & $\mathrm{CH}_{2}$ & 4-MeO-Ph & 91 & 42 & 2 cao & pyrrolidine & $\mathrm{CH}_{2}$ & $\mathrm{Bn}$ & 81 \\
\hline 3 & 2 aac & morpholine & $\mathrm{CH}_{2}$ & 4-CN-Ph & 88 & 43 & 2daa & azepine & $\mathrm{CH}_{2}$ & $\mathrm{Ph}$ & 87 \\
\hline 4 & 2aad & morpholine & $\mathrm{CH}_{2}$ & 3-thiophenyl & 187 & 44 & $2 \mathrm{dab}$ & azepine & $\mathrm{CH}_{2}$ & 4-MeO-Ph & 78 \\
\hline 5 & 2aae & morpholine & $\mathrm{CH}_{2}$ & 2-pyridyl & 80 & 45 & 2dac & azepine & $\mathrm{CH}_{2}$ & 4-CN-Ph & 88 \\
\hline 6 & 2aag & morpholine & $\mathrm{CH}_{2}$ & 3-MeO-Ph & 89 & 46 & 2dad & azepine & $\mathrm{CH}_{2}$ & 3-thiophenyl & 89 \\
\hline 7 & 2aah & morpholine & $\mathrm{CH}_{2}$ & 2-MeO-Ph & 88 & 47 & 2dae & azepine & $\mathrm{CH}_{2}$ & 2-pyridyl & 78 \\
\hline 8 & 2aai & morpholine & $\mathrm{CH}_{2}$ & 4-Me-Ph & 86 & 48 & 2dag & azepine & $\mathrm{CH}_{2}$ & 3-MeO-Ph & 84 \\
\hline 9 & 2aaj & morpholine & $\mathrm{CH}_{2}$ & 3-Me-Ph & 89 & 49 & 2dah & azepine & $\mathrm{CH}_{2}$ & 2-MeO-Ph & 80 \\
\hline 10 & 2aak & morpholine & $\mathrm{CH}_{2}$ & 2-Me-Ph & 88 & 50 & 2dai & azepine & $\mathrm{CH}_{2}$ & 4-Me-Ph & 88 \\
\hline 11 & 2aal & morpholine & $\mathrm{CH}_{2}$ & $4-\mathrm{NMe}_{2}-\mathrm{Ph}$ & 81 & 51 & 2daj & azepine & $\mathrm{CH}_{2}$ & 3-Me-Ph & 83 \\
\hline 12 & 2aam & morpholine & $\mathrm{CH}_{2}$ & 4-PhO-Ph & 94 & 52 & 2dak & azepine & $\mathrm{CH}_{2}$ & 2-Me-Ph & 78 \\
\hline 13 & 2aan & morpholine & $\mathrm{CH}_{2}$ & 2,4-di-F-Ph & 87 & 53 & 2dal & azepine & $\mathrm{CH}_{2}$ & 4-NMe $-\mathrm{Ph}$ & 82 \\
\hline 14 & 2 aao & morpholine & $\mathrm{CH}_{2}$ & $\mathrm{Bn}$ & 89 & 54 & 2dam & azepine & $\mathrm{CH}_{2}$ & 4-PhO-Ph & 90 \\
\hline 15 & 2 baa & piperidine & $\mathrm{CH}_{2}$ & $\mathrm{Ph}$ & 93 & 55 & 2dan & azepine & $\mathrm{CH}_{2}$ & 2,4-di-F-Ph & 89 \\
\hline 16 & $2 \mathrm{bab}$ & piperidine & $\mathrm{CH}_{2}$ & 4-MeO-Ph & 80 & 56 & 2dao & azepine & $\mathrm{CH}_{2}$ & $\mathrm{Bn}$ & 86 \\
\hline 17 & $2 \mathrm{bac}$ & piperidine & $\mathrm{CH}_{2}$ & 4-CN-Ph & 92 & 57 & 2eaa & NPhMe & $\mathrm{CH}_{2}$ & $\mathrm{Ph}$ & 95 \\
\hline 18 & 2bad & piperidine & $\mathrm{CH}_{2}$ & 3-thiophenyl & 193 & 58 & $2 e a b$ & NPhMe & $\mathrm{CH}_{2}$ & 4-MeO-Ph & 95 \\
\hline 19 & 2 bae & piperidine & $\mathrm{CH}_{2}$ & 2-pyridyl & 95 & 59 & 2eac & NPhMe & $\mathrm{CH}_{2}$ & 4-CN-Ph & 95 \\
\hline 20 & 2 bag & piperidine & $\mathrm{CH}_{2}$ & 3-MeO-Ph & 97 & 60 & 2ead & NPhMe & $\mathrm{CH}_{2}$ & 3-thiophenyl & 85 \\
\hline 21 & $2 \mathrm{bah}$ & piperidine & $\mathrm{CH}_{2}$ & 2-MeO-Ph & 88 & 61 & 2eae & $\mathrm{NPhMe}$ & $\mathrm{CH}_{2}$ & 2-pyridyl & 95 \\
\hline 22 & 2 bai & piperidine & $\mathrm{CH}_{2}$ & 4-Me-Ph & 97 & 62 & 2eag & NPhMe & $\mathrm{CH}_{2}$ & 3-MeO-Ph & 89 \\
\hline 23 & 2 baj & piperidine & $\mathrm{CH}_{2}$ & 3-Me-Ph & 93 & 63 & 2eah & NPhMe & $\mathrm{CH}_{2}$ & 2-MeO-Ph & 90 \\
\hline 24 & 2bak & piperidine & $\mathrm{CH}_{2}$ & 2-Me-Ph & 86 & 64 & 2eai & NPhMe & $\mathrm{CH}_{2}$ & 4-Me-Ph & 95 \\
\hline 25 & $2 \mathrm{bal}$ & piperidine & $\mathrm{CH}_{2}$ & $4-\mathrm{NMe}_{2}-\mathrm{Ph}$ & 84 & 65 & 2eaj & NPhMe & $\mathrm{CH}_{2}$ & 3-Me-Ph & 93 \\
\hline 26 & $2 \mathrm{bam}$ & piperidine & $\mathrm{CH}_{2}$ & 4-PhO-Ph & 93 & 66 & 2eak & NPhMe & $\mathrm{CH}_{2}$ & 2-Me-Ph & 94 \\
\hline 27 & 2 ban & piperidine & $\mathrm{CH}_{2}$ & 2,4-di-F-Ph & 93 & 67 & 2aca & morpholine & $\mathrm{CH}_{2} \mathrm{CH}_{2}$ & $\mathrm{Ph}$ & 84 \\
\hline 28 & 2 bao & piperidine & $\mathrm{CH}_{2}$ & $\mathrm{Bn}$ & 89 & 68 & $2 a c b$ & morpholine & $\mathrm{CH}_{2} \mathrm{CH}_{2}$ & 4-MeO-Ph & 80 \\
\hline 29 & 2 caa & pyrrolidine & $\mathrm{CH}_{2}$ & $\mathrm{Ph}$ & 81 & 69 & 2 acc & morpholine & $\mathrm{CH}_{2} \mathrm{CH}_{2}$ & 4-CN-Ph & 80 \\
\hline 30 & $2 c a b$ & pyrrolidine & $\mathrm{CH}_{2}$ & 4-MeO-Ph & 77 & 70 & 2acd & morpholine & $\mathrm{CH}_{2} \mathrm{CH}_{2}$ & 3-thiophenyl & 88 \\
\hline 31 & 2 cac & pyrrolidine & $\mathrm{CH}_{2}$ & 4-CN-Ph & 81 & 71 & 2ace & morpholine & $\mathrm{CH}_{2} \mathrm{CH}_{2}$ & 2-pyridyl & 79 \\
\hline 32 & 2 cad & pyrrolidine & $\mathrm{CH}_{2}$ & 3-thiophenyl & 176 & 72 & 2 acg & morpholine & $\mathrm{CH}_{2} \mathrm{CH}_{2}$ & 3-MeO-Ph & 88 \\
\hline 33 & 2 cae & pyrrolidine & $\mathrm{CH}_{2}$ & 2-pyridyl & 75 & 73 & 2ach & morpholine & $\mathrm{CH}_{2} \mathrm{CH}_{2}$ & 2-MeO-Ph & 88 \\
\hline 34 & 2 cag & pyrrolidine & $\mathrm{CH}_{2}$ & 3-MeO-Ph & 80 & 74 & 2 aci & morpholine & $\mathrm{CH}_{2} \mathrm{CH}_{2}$ & 4-Me-Ph & 83 \\
\hline 35 & $2 \mathrm{cah}$ & pyrrolidine & $\mathrm{CH}_{2}$ & 2-MeO-Ph & 77 & 75 & $2 a c j$ & morpholine & $\mathrm{CH}_{2} \mathrm{CH}_{2}$ & 3-Me-Ph & 88 \\
\hline 36 & 2 cai & pyrrolidine & $\mathrm{CH}_{2}$ & 4-Me-Ph & 83 & 76 & 2ack & morpholine & $\mathrm{CH}_{2} \mathrm{CH}_{2}$ & 2-Me-Ph & 80 \\
\hline 37 & 2caj & pyrrolidine & $\mathrm{CH}_{2}$ & 3-Me-Ph & 83 & 77 & $2 \mathrm{acl}$ & morpholine & $\mathrm{CH}_{2} \mathrm{CH}_{2}$ & $4-\mathrm{NMe}_{2}-\mathrm{Ph}$ & 88 \\
\hline 38 & 2cak & pyrrolidine & $\mathrm{CH}_{2}$ & 2-Me-Ph & 79 & 78 & $2 \mathrm{acm}$ & morpholine & $\mathrm{CH}_{2} \mathrm{CH}_{2}$ & 4-PhO-Ph & 88 \\
\hline 39 & $2 \mathrm{cal}$ & pyrrolidine & $\mathrm{CH}_{2}$ & $4-\mathrm{NMe}_{2}-\mathrm{Ph}$ & 81 & 79 & 2acn & morpholine & $\mathrm{CH}_{2} \mathrm{CH}_{2}$ & 2,4-di-F-Ph & 82 \\
\hline 40 & 2cam & pyrrolidine & $\mathrm{CH}_{2}$ & 4-PhO-Ph & 86 & 80 & 2aco & morpholine & $\mathrm{CH}_{2} \mathrm{CH}_{2}$ & $\mathrm{Bn}$ & 88 \\
\hline
\end{tabular}

\section{Experimental Section}

\subsection{General}

All chemicals were reagent grade and used as purchased. The Merrifield resin (loading capacity $1.29 \mathrm{mmol} / \mathrm{g}, 100-200 \mathrm{mesh}$ ) was purchased from BeadTech (Seoul, Korea). Reactions were monitored by TLC analysis using silica gel 60 F-254 thin layer plates (Merck, Darmstadt, Germany) or ATR-FRIR analysis using a Cary 630 instrument (Agilent Technologies, Santa Clara, CA, USA). Flash column chromatography was carried out on Merck silica gel 60 (230-400 mesh). The crude products were purified by parallel chromatography using CombiFlash (Isco, Lincoln, NE, USA). ${ }^{1} \mathrm{H}-\mathrm{NMR}$ $(500 \mathrm{MHz})$ and ${ }^{13} \mathrm{C}-\mathrm{NMR}(125 \mathrm{MHz})$ spectra were recorded in $\delta$ units relative to deuterated solvent $\left(\mathrm{CDCl}_{3}, \mathrm{DMSO}-d_{6}\right.$, etc.) as internal reference on a $500 \mathrm{MHz}$ NMR instrument (Bruker, Billerca, MA, USA). LC-MS analysis was performed on ESI mass spectrometer with PDA detection. LC-MS area\% 
purities of all products were determined by LC peak area analysis (XBD C18 column, $4.6 \mathrm{~mm} \times 100 \mathrm{~mm}$; PDA detector at $200-400 \mathrm{~nm}$; isocratic, $5 \mathrm{mM}$ ammonium formate $/ \mathrm{CH}_{3} \mathrm{CN}(30: 70)$ ).

\subsection{General Procedure for the Preparation of Secondary $\alpha-1,2,3$-Triazoloamides 1 on Solid-Phase}

A typical procedure for the desired secondary $\alpha-1,2,3$-triazoloamides 1, as exemplified for $\mathrm{N}$-phenyl-2-(4-phenyl-1H-1,2,3-triazol-1-yl)acetamide (1aaa; $\mathrm{R}^{1}=\mathrm{R}^{3}=\mathrm{Ph}, \mathbf{A}=\mathrm{CH}_{2}$ ) follows.

\subsubsection{Preparation of AMEBA Resin 4}

Merrifield resin $(53.2 \mathrm{~g}, 50.0 \mathrm{mmol}, 0.94 \mathrm{mmol} / \mathrm{g}$ ) was treated with 4-formyl-3-methoxyphenol $(22.8 \mathrm{~g}, 150.0 \mathrm{mmol})$, potassium iodide $(83.0 \mathrm{mg}, 0.5 \mathrm{mmol})$, and potassium carbonate $(20.7 \mathrm{~g}$, $150.0 \mathrm{mmol})$ in DMF (300 mL). The mixture was shaken at room temperature for $10 \mathrm{~h}$, and then filtered, washed several times with $\mathrm{H}_{2} \mathrm{O}, \mathrm{DMF}, \mathrm{MeOH}$, and $\mathrm{CH}_{2} \mathrm{Cl}_{2}$, and dried in a vacuo to give AMEBA resin 4 (59.0 g): On-bead ATR-FTIR (neat) $v_{\max } 1678,1598,1259\left(\mathrm{~cm}^{-1}\right)$.

\subsubsection{Preparation of Secondary Amine Resin 3}

A mixture of AMEBA resin $4(10 \mathrm{~g}$, theoretically $8.5 \mathrm{mmol})$, aniline $(5 \mathbf{a} ;(2.3 \mathrm{~mL}, 25.5 \mathrm{mmol})$, sodium triacetoxyborohydride $(5.4 \mathrm{~g}, 25.5 \mathrm{mmol})$, and acetic acid $(0.49 \mathrm{~mL}, 8.5 \mathrm{mmol})$ in 1,2-dichloroethane was heated at $50{ }^{\circ} \mathrm{C}$ for $12 \mathrm{~h}$. The reaction mixture was cooled to room temperature, and then filtered, washed several times with $\mathrm{H}_{2} \mathrm{O}, \mathrm{DMF}, \mathrm{MeOH}$, and $\mathrm{CH}_{2} \mathrm{Cl}_{2}$, and dried in a vacuum oven to give secondary amine resin $3 \mathbf{a}(10.6 \mathrm{~g})$ : On-bead ATR-FTIR (neat) $v_{\max } 3424$, $1597,1489\left(\mathrm{~cm}^{-1}\right)$.

\subsubsection{Preparation of $\alpha$-Chloroamide Resin 7}

The amine resin $3 \mathrm{a}(3.0 \mathrm{~g}$, theoretically $2.4 \mathrm{mmol})$ was treated with 2-chloroacetyl chloride (6a; $0.57 \mathrm{~mL}, 7.2 \mathrm{mmol})$ and triethylamine $(1.0 \mathrm{~mL}, 7.2 \mathrm{mmol})$ in $\mathrm{CH}_{2} \mathrm{Cl}_{2}$ at $0{ }^{\circ} \mathrm{C}$. The reaction mixture was shaken at room temperature for $5 \mathrm{~h}$, and then filtered, washed several times with $\mathrm{H}_{2} \mathrm{O}, \mathrm{DMF}$, $\mathrm{MeOH}$, and $\mathrm{CH}_{2} \mathrm{Cl}_{2}$, and dried in a vacuum oven to give $\alpha$-chloroamide resin $7 \mathbf{a a}(3.16 \mathrm{~g})$ : On-bead ATR-FTIR (neat) $v_{\max } 1666,1593,1489\left(\mathrm{~cm}^{-1}\right)$.

\subsubsection{Preparation of $\alpha$-Azidoamide Resin 8}

The $\alpha$-chloroamide resin 7 aa $(2.8 \mathrm{~g}$, theoretically $2.1 \mathrm{mmol})$ was treated with sodium azide $(0.47 \mathrm{~g}, 7.2 \mathrm{mmol})$ in DMF. The reaction mixture was shaken at room temperature for $12 \mathrm{~h}$, and then filtered, washed several times with $\mathrm{H}_{2} \mathrm{O}, \mathrm{DMF}, \mathrm{MeOH}$, and $\mathrm{CH}_{2} \mathrm{Cl}_{2}$, and dried in a vacuum oven to give $\alpha$-azidoamide resin 8aa (2.8 g): On-bead ATR-FTIR (neat) $v_{\max } 2101,1670,1590,1491\left(\mathrm{~cm}^{-1}\right)$.

\subsubsection{Preparation of $\alpha-1,2,3$-Triazoloamide Resin $\mathbf{1 0}$}

To a mixture of $\alpha$-azidoamide resin $8 \mathbf{a a}(570 \mathrm{mg}$, theoretically $0.42 \mathrm{mmol})$ and phenylacetylene (9a, $0.07 \mathrm{~mL}, 0.6 \mathrm{mmol})$ in DMF / piperidine (4:1) was added copper(I) iodide (229 mg, $1.27 \mathrm{mmol}$ ) and sodium ascorbate $(57 \mathrm{mg}, 1.27 \mathrm{mmol}$ ) at room temperature. The reaction mixture was shaken at room temperature for $12 \mathrm{~h}$, and then filtered, washed several times with $\mathrm{H}_{2} \mathrm{O}, \mathrm{DMF}, \mathrm{MeOH}$, and $\mathrm{CH}_{2} \mathrm{Cl}_{2}$, and dried in a vacuum oven to give $\alpha-1,2,3$-triazoloamide resin 10aaa $(606 \mathrm{mg})$ : On-bead ATR-FTIR (neat) $v_{\max } 1669,1590,1489\left(\mathrm{~cm}^{-1}\right)$.

\subsubsection{Preparation of $\alpha-1,2,3$-Triazoloamide 1}

The $\alpha$-1,2,3-triazoloamide resin 10aaa (157 $\mathrm{mg}$, theoretically $0.10 \mathrm{mmol}$ ) was added $30 \%$ TFA in $\mathrm{CH}_{2} \mathrm{Cl}_{2}(3 \mathrm{~mL})$. The reaction mixture was stirred at $45^{\circ} \mathrm{C}$ for 1day and the mixture was filtered and washed with $\mathrm{CH}_{2} \mathrm{Cl}_{2}$ and $\mathrm{MeOH}$. The filtrate was evaporated in vacuo and the residue was dissolved in $\mathrm{CH}_{2} \mathrm{Cl}_{2}$ and extracted with saturated $\mathrm{NaHCO}_{3}$. The aqueous layer was extracted with $\mathrm{CH}_{2} \mathrm{Cl}_{2}$ twice and the combined organic extracts were washed with brine, dried over $\mathrm{MgSO}_{4}$, filtered 
and concentrated in vacuo to give the target $\mathrm{N}$-phenyl-2-(4-phenyl-1H-1,2,3-triazol-1-yl)acetamide (1aaa) was obtained as a light yellow solid (23 mg, 93\% from Merrifield resin). Mp 247-249 ${ }^{\circ} \mathrm{C}$; ${ }^{1} \mathrm{H}-\mathrm{NMR}\left(\mathrm{DMSO}-d_{6}\right) \delta 5.39(\mathrm{~s}, 2 \mathrm{H}), 7.09(\mathrm{~m}, 1 \mathrm{H}), 7.33-7.36(\mathrm{~m}, 3 \mathrm{H}), 7.46(\mathrm{~m}, 2 \mathrm{H}), 7.60(\mathrm{~m}, 2 \mathrm{H}), 7.88$ (m, 2H), $8.60(\mathrm{~s}, 1 \mathrm{H}), 10.51(\mathrm{~s}, 1 \mathrm{H}) ;{ }^{13} \mathrm{C}-\mathrm{NMR}\left(\mathrm{DMSO}-d_{6}\right) \delta 52.4,119.3,123.1,123.8,125.1,127.9,128.9$, 130.7, 138.4, 146.2, 164.2; IR (ATR) $v_{\max } 3273,3061,1677,1601,1545,1442,1365,1253,1202,1080$, $753\left(\mathrm{~cm}^{-1}\right)$; LC-MS (ESI) $\mathrm{m} / z 279\left([\mathrm{M}+1]^{+}\right)$; HRMS (FAB) calcd for $\mathrm{C}_{16} \mathrm{H}_{15} \mathrm{~N}_{4} \mathrm{O}\left([\mathrm{M}+\mathrm{H}]^{+}\right)$279.1240, found 279.1239 .

\subsection{Characterization Data of Secondary $\alpha-1,2,3$-Triazoloamides 1}

2-[4-(4-Methoxyphenyl)-1H-1,2,3-triazol-1-yl]-N-phenylacetamide (1aab). White solid; Yield: 91\%. ${ }^{1} \mathrm{H}-\mathrm{NMR}$ (DMSO- $\left.d_{6}\right) \delta 3.79(\mathrm{~s}, 3 \mathrm{H}), 5.36(\mathrm{~s}, 2 \mathrm{H}), 7.03(\mathrm{~d}, J=8.9 \mathrm{~Hz}, 2 \mathrm{H}), 7.09(\mathrm{~m}, 1 \mathrm{H}), 7.35(\mathrm{~m}, 2 \mathrm{H}), 7.60$ $(\mathrm{m}, 2 \mathrm{H}), 7.80(\mathrm{~d}, J=8.8 \mathrm{~Hz}, 2 \mathrm{H}), 8.48(\mathrm{~s}, 1 \mathrm{H}), 10.50(\mathrm{~s}, 1 \mathrm{H}) ;{ }^{13} \mathrm{C}-\mathrm{NMR}$ (DMSO- $\left.d_{6}\right) \delta 52.3,55.2,114.4$, $119.3,122.1,123.3,123.8,126.5,128.9,138.4,146.2,159.0,164.2$; LC-MS (ESI) $m / z 309\left([\mathrm{M}+1]^{+}\right)$.

2-[4-(4-Cyanophenyl)-1H-1,2,3-triazol-1-yl]-N-phenylacetamide (1aac). Light brown solid; Yield: 83\%. ${ }^{1} \mathrm{H}-\mathrm{NMR}\left(\right.$ DMSO- $\left.d_{6}\right) \delta 5.43(\mathrm{~s}, 2 \mathrm{H}), 7.09(\mathrm{~m}, 1 \mathrm{H}), 7.34(\mathrm{~m}, 2 \mathrm{H}), 7.59(\mathrm{~m}, 2 \mathrm{H}), 7.93(\mathrm{~d}, J=8.6 \mathrm{~Hz}$, $2 \mathrm{H}), 8.09(\mathrm{~d}, J=8.5 \mathrm{~Hz}, 2 \mathrm{H}), 8.82(\mathrm{~s}, 1 \mathrm{H}), 10.53(\mathrm{~s}, 1 \mathrm{H}) ;{ }^{13} \mathrm{C}-\mathrm{NMR}\left(\mathrm{DMSO}-d_{6}\right) \delta 52.5,110.1,118.8,119.2$, 123.8, 124.8, 125.7, 128.9, 133.0, 135.2, 138.4, 144.7, 164.0; LC-MS (ESI) $\mathrm{m} / z 304\left([\mathrm{M}+1]^{+}\right)$.

N-Phenyl-2-[4-(thiophen-3-yl)-1H-1,2,3-triazol-1-yl]acetamide (1aad). White solid; Yield: 82\%. ${ }^{1} \mathrm{H}-\mathrm{NMR}$ $\left(\right.$ DMSO- $\left._{6}\right) \delta 5.37(\mathrm{~s}, 2 \mathrm{H}), 7.09(\mathrm{~m}, 1 \mathrm{H}), 7.34(\mathrm{~m}, 2 \mathrm{H}), 7.55(\mathrm{dd}, J=1.2,5.0 \mathrm{~Hz}, 1 \mathrm{H}), 7.59(\mathrm{~m}, 2 \mathrm{H}), 7.66$ $(\mathrm{dd}, J=3.0,5.0 \mathrm{~Hz}, 1 \mathrm{H}), 7.88(\mathrm{dd}, J=1.2,2.9 \mathrm{~Hz}, 1 \mathrm{H}), 8.46(\mathrm{~s}, 1 \mathrm{H}), 10.50(\mathrm{~s}, 1 \mathrm{H}) ;{ }^{13} \mathrm{C}-\mathrm{NMR}$ (DMSO- $d_{6}$ ) $\delta 52.3,119.2,120.8,122.8,123.8,125.8,127.1,128.9,132.0,138.4,142.8,164.2$; LC-MS (ESI) $\mathrm{m} / z 285$ $\left([\mathrm{M}+1]^{+}\right)$.

N-Phenyl-2-[4-(pyridin-2-yl)-1H-1,2,3-triazol-1-yl]acetamide (1aae). White solid; Yield: $85 \% .{ }^{1} \mathrm{H}-\mathrm{NMR}$ $\left(\right.$ DMSO- $\left.d_{6}\right) \delta 5.42(\mathrm{~s}, 2 \mathrm{H}), 7.09(\mathrm{~m}, 1 \mathrm{H}), 7.33-7.36(\mathrm{~m}, 3 \mathrm{H}), 7.59(\mathrm{~m}, 2 \mathrm{H}), 7.91(\mathrm{t}, J=7.7 \mathrm{~Hz}, 1 \mathrm{H}), 8.06(\mathrm{~d}$, $J=7.6 \mathrm{~Hz}, 1 \mathrm{H}), 8.62(\mathrm{~s}, 2 \mathrm{H}), 10.51(\mathrm{~s}, 1 \mathrm{H}) ;{ }^{13} \mathrm{C}-\mathrm{NMR}\left(\mathrm{DMSO}-d_{6}\right) \delta 52.3,119.2,119.4,123.0,123.8,125.0$, 128.9, 137.2, 138.4, 147.1, 149.7, 150.0, 164.1; LC-MS (ESI) $m / z 280\left([\mathrm{M}+1]^{+}\right)$.

2-(4-Butyl-1H-1,2,3-triazol-1-yl)-N-phenylacetamide (1aaf). White solid; Yield: 92\%. ${ }^{1} \mathrm{H}-\mathrm{NMR}$ $\left(\mathrm{DMSO}_{6} d_{6}\right) 0.90(\mathrm{t}, J=7.3 \mathrm{~Hz}, 3 \mathrm{H}), 1.34(\mathrm{~m}, 2 \mathrm{H}), 1.59(\mathrm{~m}, 2 \mathrm{H}), 2.64(\mathrm{~m}, 2 \mathrm{H}), 5.26(\mathrm{~s}, 2 \mathrm{H}), 7.08(\mathrm{~m}$, 1H), $7.33(\mathrm{~m}, 2 \mathrm{H}), 7.58(\mathrm{~m}, 2 \mathrm{H}), 7.86(\mathrm{~s}, 1 \mathrm{H}), 10.43(\mathrm{~s}, 1 \mathrm{H}) ;{ }^{13} \mathrm{C}-\mathrm{NMR}$ (DMSO-d 6 ) $\delta 13.7,21.7,24.6,31.1$, $52.1,119.2,123.4,123.7,128.9,138.4,146.7,164.4$; LC-MS (ESI) $m / z 259\left([\mathrm{M}+1]^{+}\right)$.

N-(4-Methoxyphenyl)-2-(4-phenyl-1H-1,2,3-triazol-1-yl)acetamide (1baa). White solid; Yield: 92\%. ${ }^{1} \mathrm{H}-\mathrm{NMR}\left(\mathrm{DMSO}-d_{6}\right) \delta 3.72(\mathrm{~s}, 3 \mathrm{H}), 5.34(\mathrm{~s}, 2 \mathrm{H}), 6.91(\mathrm{~d}, J=9.1 \mathrm{~Hz}, 2 \mathrm{H}), 7.34(\mathrm{~m}, 1 \mathrm{H}), 7.44-7.47$ $(\mathrm{m}, 2 \mathrm{H}), 7.51(\mathrm{~d}, J=9.1 \mathrm{~Hz}, 2 \mathrm{H}), 7.87-7.88(\mathrm{~m}, 2 \mathrm{H}), 8.59(\mathrm{~s}, 1 \mathrm{H}), 10.37(\mathrm{~s}, 1 \mathrm{H}) ;{ }^{13} \mathrm{C}-\mathrm{NMR}\left(\mathrm{DMSO}-d_{6}\right) \delta$ 52.3, 55.2, 114.1, 120.9, 123.1, 125.2, 127.9, 129.0, 130.7, 131.5, 146.2, 155.6, 163.7; LC-MS (ESI) m/z 309 $\left([\mathrm{M}+1]^{+}\right)$.

N-(4-Methoxyphenyl)-2-[4-(4-methoxyphenyl)-1H-1,2,3-triazol-1-yl]acetamide (1bab). Yellow solid; Yield: 94\%. ${ }^{1} \mathrm{H}-\mathrm{NMR}\left(\mathrm{DMSO}-d_{6}\right) \delta 3.72(\mathrm{~s}, 3 \mathrm{H}), 3.79(\mathrm{~s}, 3 \mathrm{H}), 5.31(\mathrm{~s}, 2 \mathrm{H}), 6.91(\mathrm{~d}, J=9.1 \mathrm{~Hz}, 2 \mathrm{H}), 7.02(\mathrm{~d}$, $J=8.9 \mathrm{~Hz}, 2 \mathrm{H}), 7.51(\mathrm{~d}, J=9.1 \mathrm{~Hz}, 2 \mathrm{H}), 7.79(\mathrm{~d}, J=8.9 \mathrm{~Hz}, 2 \mathrm{H}), 8.47(\mathrm{~s}, 1 \mathrm{H}), 10.36(\mathrm{~s}, 1 \mathrm{H}) ;{ }^{13} \mathrm{C}-\mathrm{NMR}$ (DMSO- $d_{6}$ ) $\delta 52.3,55.18,55.21,114.1,114.4,120.9,122.1,123.4,126.5,131.5,155.6,158.0,159.0,163.7$; LC-MS (ESI) $m / z 339\left([\mathrm{M}+1]^{+}\right)$.

2-[4-(4-Cyanophenyl)-1H-1,2,3-triazol-1-yl]-N-(4-methoxyphenyl)acetamide (1bac). Yellow solid; Yield: 89\%. ${ }^{1} \mathrm{H}-\mathrm{NMR}\left(\mathrm{DMSO}-d_{6}\right) \delta 3.72(\mathrm{~s}, 3 \mathrm{H}), 5.38(\mathrm{~s}, 2 \mathrm{H}), 6.91(\mathrm{~d}, J=9.1 \mathrm{~Hz}, 2 \mathrm{H}), 7.50(\mathrm{~d}, J=9.1 \mathrm{~Hz}$, $2 \mathrm{H}), 7.93(\mathrm{~d}, J=8.6 \mathrm{~Hz}, 2 \mathrm{H}), 8.08(\mathrm{~d}, J=8.6 \mathrm{~Hz}, 2 \mathrm{H}), 8.81(\mathrm{~s}, 1 \mathrm{H}), 10.39(\mathrm{~s}, 1 \mathrm{H}) ;{ }^{13} \mathrm{C}-\mathrm{NMR}\left(\mathrm{DMSO}-d_{6}\right)$ $\delta 52.9,55.7,110.6,114.5,119.3,121.3,125.2,126.2,131.9,133.5,135.6,145.1,156.1,163.9$; LC-MS (ESI) $\mathrm{m} / \mathrm{z} 334\left([\mathrm{M}+1]^{+}\right)$.

N-(4-Methoxyphenyl)-2-[4-(thiophen-3-yl)-1H-1,2,3-triazol-1-yl]acetamide (1bad). Light brown solid; Yield: 85\%. ${ }^{1} \mathrm{H}-\mathrm{NMR}\left(\mathrm{DMSO}_{6}\right) \delta 3.72(\mathrm{~s}, 3 \mathrm{H}), 5.33(\mathrm{~s}, 2 \mathrm{H}), 6.91(\mathrm{~d}, J=9.1 \mathrm{~Hz}, 2 \mathrm{H}), 7.51(\mathrm{~d}$, 
$J=9.1 \mathrm{~Hz}, 2 \mathrm{H}) 7.55(\mathrm{dd}, J=1.3,5.0 \mathrm{~Hz}, 1 \mathrm{H}), 7.65(\mathrm{dd}, J=3.0,5.0 \mathrm{~Hz}, 1 \mathrm{H}), 7.88(\mathrm{dd}, J=1.2,2.9 \mathrm{~Hz}$, $1 \mathrm{H}), 8.45(\mathrm{~s}, 1 \mathrm{H}), 10.36(\mathrm{~s}, 1 \mathrm{H}),{ }^{13} \mathrm{C}-\mathrm{NMR}\left(\mathrm{DMSO}-d_{6}\right) \delta 52.2,55.2,114.0,120.8,122.7,125.8,127.1,131.5$, 132.0, 142.8, 155.6, 163.6; LC-MS (ESI) $m / z 315\left([\mathrm{M}+1]^{+}\right)$.

N-(4-Methoxyphenyl)-2-[4-(3-methoxyphenyl)-1H-1,2,3-triazol-1-yl]acetamide (1bag). White solid; Yield: 87\%. ${ }^{1} \mathrm{H}-\mathrm{NMR}\left(\mathrm{CDCl}_{3}\right) \delta 3.78(\mathrm{~s}, 3 \mathrm{H}), 3.88(\mathrm{~s}, 3 \mathrm{H}), 5.23(\mathrm{~s}, 2 \mathrm{H}), 6.85(\mathrm{~d}, J=9.1 \mathrm{~Hz}, 2 \mathrm{H}), 6.93(\mathrm{ddd}$, $J=1.3,2.5,7.9 \mathrm{~Hz}, 1 \mathrm{H}), 7.34-7.40(\mathrm{~m}, 4 \mathrm{H}), 7.46(\mathrm{~m}, 1 \mathrm{H}), 7.78(\mathrm{br} \mathrm{s}, 1 \mathrm{H}), 7.97(\mathrm{~s}, 1 \mathrm{H}),{ }^{13} \mathrm{C}-\mathrm{NMR}$ $\left(\right.$ DMSO- $d_{6}$ ) $\delta 52.3,55.1,55.2,110.3,113.6,114.0,117.5,120.8,123.2,130.0,131.5,132.1,146.1,155.6$, 159.7, 163.6; LC-MS (ESI) $m / z 339\left([\mathrm{M}+1]^{+}\right)$.

N-(4-Methoxyphenyl)-2-(4-m-tolyl-1H-1,2,3-triazol-1-yl)acetamide (1baj). White solid; Yield: 89\%. ${ }^{1} \mathrm{H}-\mathrm{NMR}\left(\mathrm{CDCl}_{3}\right) \delta 2.42(\mathrm{~s}, 3 \mathrm{H}), 3.78(\mathrm{~s}, 3 \mathrm{H}), 5.22(\mathrm{~s}, 2 \mathrm{H}), 6.85(\mathrm{~d}, J=9.1 \mathrm{~Hz}, 2 \mathrm{H}), 7.19(\mathrm{~d}, J=7.6 \mathrm{~Hz}$, $1 \mathrm{H}), 7.34(\mathrm{t}, J=7.7 \mathrm{~Hz}, 1 \mathrm{H}), 7.36(\mathrm{~d}, J=9.1 \mathrm{~Hz}, 2 \mathrm{H}), 7.63(\mathrm{~d}, J=7.7 \mathrm{~Hz}, 1 \mathrm{H}), 7.71(\mathrm{~s}, 1 \mathrm{H}), 7.83(\mathrm{br} \mathrm{s}, 1 \mathrm{H})$, $7.96(\mathrm{~s}, 1 \mathrm{H}) ;{ }^{13} \mathrm{C}-\mathrm{NMR}\left(\mathrm{DMSO}-d_{6}\right) \delta$ 21.0, 52.3, 55.2, 114.0, 120.8, 122.3, 122.9, 125.7, 128.5, 128.8, 130.6, 131.5, 138.0, 146.3, 155.6, 163.6;LC-MS (ESI) $\mathrm{m} / z 323\left([\mathrm{M}+1]^{+}\right)$.

N-(4-Methoxyphenyl)-2-(4-o-tolyl-1H-1,2,3-triazol-1-yl)acetamide (1bak). Light yellow solid; Yield: 84\%. ${ }^{1} \mathrm{H}-\mathrm{NMR}\left(\mathrm{CDCl}_{3}\right) \delta 2.50(\mathrm{~s}, 3 \mathrm{H}), 3.79(\mathrm{~s}, 3 \mathrm{H}), 5.25(\mathrm{~s}, 2 \mathrm{H}), 6.86(\mathrm{~d}, J=9.1 \mathrm{~Hz}, 2 \mathrm{H}), 7.30-7.32(\mathrm{~m}, 3 \mathrm{H})$, $7.38(\mathrm{~d}, J=9.0 \mathrm{~Hz}, 2 \mathrm{H}), 7.80(\mathrm{~m}, 1 \mathrm{H}), 7.86(\mathrm{br} \mathrm{s}, 1 \mathrm{H}), 7.87(\mathrm{~s}, 1 \mathrm{H}) ;{ }^{13} \mathrm{C}-\mathrm{NMR}$ (DMSO- $\left.d_{6}\right) \delta 21.1,52.2$, 55.2, 114.0, 120.8, 124.9, 126.0, 127.8, 128.1, 130.0, 130.9, 131.5, 134.8, 145.3, 155.6, 163.7; LC-MS (ESI) $m / z 323\left([\mathrm{M}+1]^{+}\right)$.

2-\{4-[4-(Dimethylamino)phenyl]-1H-1,2,3-triazol-1-yl\}-N-(4-methoxyphenyl)acetamide (1bal). Brown solid; Yield: 79\%. ${ }^{1} \mathrm{H}-\mathrm{NMR}\left(\mathrm{DMSO}-d_{6}\right) \delta 2.94(\mathrm{~s}, 6 \mathrm{H}), 3.72(\mathrm{~s}, 3 \mathrm{H}), 5.32(\mathrm{~s}, 2 \mathrm{H}), 6.68(\mathrm{dd}, J=1.9,8.3 \mathrm{~Hz}, 1 \mathrm{H})$, $6.79(\mathrm{~d}, J=9.0 \mathrm{~Hz}, 2 \mathrm{H}), 7.11(\mathrm{dd}, J=1.0,8.1 \mathrm{~Hz}, 1 \mathrm{H}), 7.24(\mathrm{t}, J=8.2 \mathrm{~Hz}, 1 \mathrm{H}), 7.30(\mathrm{t}, J=2.1 \mathrm{~Hz}, 1 \mathrm{H})$, $7.67(\mathrm{~d}, J=8.9 \mathrm{~Hz}, 2 \mathrm{H}), 8.36(\mathrm{~s}, 1 \mathrm{H}), 10.48(\mathrm{~s}, 1 \mathrm{H}) ;{ }^{13} \mathrm{C}-\mathrm{NMR}\left(\mathrm{DMSO}-d_{6}\right) \delta 40.1,52.2,55.2,112.4,114.1$, $118.7,121.2,126.1,131.5,146.9,150.1,155.6,163.8$; LC-MS (ESI) $\mathrm{m} / z 352\left([\mathrm{M}+1]^{+}\right)$.

N-(4-Methoxyphenyl)-2-[4-(4-phenoxyphenyl)-1H-1,2,3-triazol-1-yl]acetamide (1bam). Light yellow solid; Yield: 92\%. ${ }^{1} \mathrm{H}-\mathrm{NMR}\left(\mathrm{DMSO}-d_{6}\right) \delta 3.72(\mathrm{~s}, 3 \mathrm{H}), 5.34(\mathrm{~s}, 2 \mathrm{H}), 6.91(\mathrm{~d}, J=9.1 \mathrm{~Hz}, 2 \mathrm{H}), 7.06-7.10(\mathrm{~m}, 4 \mathrm{H})$, $7.17(\mathrm{~m}, 1 \mathrm{H}), 7.40-7.44(\mathrm{~m}, 2 \mathrm{H}), 7.51(\mathrm{~d}, J=9.1 \mathrm{~Hz}, 2 \mathrm{H}), 7.88(\mathrm{~d}, J=8.8 \mathrm{~Hz}, 2 \mathrm{H}), 8.55(\mathrm{~s}, 1 \mathrm{H}), 10.37(\mathrm{~s}$, $1 \mathrm{H}) ;{ }^{13} \mathrm{C}-\mathrm{NMR}$ (DMSO- $d_{6}$ ) $\delta 52.3,55.2,114.1,118.9,119.0,120.9,122.7,123.7,126.1,127.0,130.2,131.5$, 145.8, 155.6, 156.5, 163.7; LC-MS (ESI) $m / z 401\left([\mathrm{M}+1]^{+}\right)$.

2-[4-(2,4-Difluorophenyl)-1H-1,2,3-triazol-1-yl]-N-(4-methoxyphenyl)acetamide (1ban). Light yellow solid; Yield: 87\%. ${ }^{1} \mathrm{H}-\mathrm{NMR}\left(\mathrm{CDCl}_{3}\right) \delta 3.78(\mathrm{~s}, 3 \mathrm{H}), 5.24(\mathrm{~s}, 2 \mathrm{H}), 6.85(\mathrm{~d}, J=9.1 \mathrm{~Hz}, 2 \mathrm{H}), 6.93(\mathrm{ddd}$, $J=2.4,8.6,11.0 \mathrm{~Hz}, 1 \mathrm{H}), 7.03(\mathrm{~m}, 1 \mathrm{H}), 7.36(\mathrm{~d}, J=9.1 \mathrm{~Hz}, 2 \mathrm{H}), 7.70(\mathrm{br} \mathrm{s}, 1 \mathrm{H}), 8.10(\mathrm{~d}, J=3.6 \mathrm{~Hz}$, $1 \mathrm{H}), 8.30(\mathrm{dt}, J=6.5,8.6 \mathrm{~Hz}, 1 \mathrm{H}) ;{ }^{13} \mathrm{C}-\mathrm{NMR}\left(\mathrm{DMSO}-d_{6}\right) \delta 52.2,55.2,104.6\left(\mathrm{t}, J_{\mathrm{CF}}=26.0 \mathrm{~Hz}\right), 112.3(\mathrm{dd}$, $\left.J_{\mathrm{CF}}=21.2,3.5 \mathrm{~Hz}\right), 115.2\left(\mathrm{dd}, J_{\mathrm{CF}}=13.3,3.6 \mathrm{~Hz}\right), 114.0,125.2\left(\mathrm{~d}, J_{\mathrm{CF}}=10.8 \mathrm{~Hz}\right), 120.8,128.5(\mathrm{dd}$, $\left.J_{\mathrm{CF}}=9.8,5.1 \mathrm{~Hz}\right), 131.5,138.8\left(\mathrm{~d}, J_{\mathrm{CF}}=2.6 \mathrm{~Hz}\right), 155.5,158.5\left(\mathrm{dd}, J_{\mathrm{CF}}=250.3,12.7 \mathrm{~Hz}\right), 161.7(\mathrm{dd}$, $\left.J_{\mathrm{CF}}=247.4,12.7 \mathrm{~Hz}\right), 163.6$; LC-MS (ESI) $m / z 345\left([\mathrm{M}+1]^{+}\right)$.

2-(4-Benzyl-1H-1,2,3-triazol-1-yl)-N-(4-methoxyphenyl)acetamide (1bao). White solid; Yield: 85\%. ${ }^{1} \mathrm{H}-\mathrm{NMR}\left(\mathrm{CDCl}_{3}\right) \delta 3.78(\mathrm{~s}, 3 \mathrm{H}), 4.13(\mathrm{~s}, 2 \mathrm{H}), 5.09(\mathrm{~s}, 2 \mathrm{H}), 6.84(\mathrm{~d}, J=9.0 \mathrm{~Hz}, 2 \mathrm{H}), 7.22-7.34(\mathrm{~m}, 7 \mathrm{H})$, 7.37 (s, $1 \mathrm{H}), 7.81$ (br s, $1 \mathrm{H}) ;{ }^{13} \mathrm{C}-\mathrm{NMR}$ (DMSO-d $\left.d_{6}\right) \delta 31.3,52.1,55.2,114.0,120.8,124.2,126.2,128.4$, 128.6, 131.5, 139.6, 145.9, 155.6, 163.8; LC-MS (ESI) $m / z 323\left([\mathrm{M}+1]^{+}\right)$.

N-(3-Methoxyphenyl)-2-(4-phenyl-1H-1,2,3-triazol-1-yl)acetamide (1caa). Light yellow solid; Yield: 91\%. ${ }^{1} \mathrm{H}-\mathrm{NMR}\left(\mathrm{CDCl}_{3}\right) \delta 3.79(\mathrm{~s}, 3 \mathrm{H}), 5.24(\mathrm{~s}, 2 \mathrm{H}), 6.70(\mathrm{dd}, J=2.2,8.1 \mathrm{~Hz}, 1 \mathrm{H}), 6.97(\mathrm{dd}, J=1.5,7.9 \mathrm{~Hz}$, $1 \mathrm{H})$, 7.19-7.23 (m, 2H), 7.37 (m, 1H), 7.44-7.47 (m, 2H), 7.85-7.87 (m, 2H), $7.95(\mathrm{~s}, 1 \mathrm{H}), 7.98(\mathrm{~s}, 1 \mathrm{H})$; ${ }^{13}$ C-NMR (DMSO- $d_{6}$ ) $\delta 52.4,55.0,105.0,109.3,111.5,123.0,125.1,127.8,128.9,129.7,130.7,139.5,146.2$, 159.6, 164.2; LC-MS (ESI) $\mathrm{m} / \mathrm{z} 309\left([\mathrm{M}+1]^{+}\right)$.

N-(3-Methoxyphenyl)-2-[4-(4-methoxyphenyl)-1H-1,2,3-triazol-1-yl]acetamide (1cab). White solid; Yield: 90\%. ${ }^{1} \mathrm{H}-\mathrm{NMR}\left(\mathrm{CDCl}_{3}\right) \delta 3.79(\mathrm{~s}, 3 \mathrm{H}), 3.86(\mathrm{~s}, 3 \mathrm{H}), 5.22(\mathrm{~s}, 2 \mathrm{H}), 6.70(\mathrm{dd}, J=2.3,7.9 \mathrm{~Hz}, 1 \mathrm{H}), 6.96-6.99$ 
$(\mathrm{m}, 3 \mathrm{H}), 7.18(\mathrm{t}, J=2.2 \mathrm{~Hz}, 1 \mathrm{H}), 7.21(\mathrm{t}, J=8.2 \mathrm{~Hz}, 1 \mathrm{H}), 7.78(\mathrm{~d}, J=8.9 \mathrm{~Hz}, 2 \mathrm{H}), 7.88(\mathrm{~s}, 1 \mathrm{H}), 7.91(\mathrm{~s}$, 1H); ${ }^{13}$ C-NMR (DMSO- $d_{6}$ ) $\delta 52.3,55.0,55.1,105.0,109.3,111.5,114.3,122.1,123.3,126.5,129.7,139.6$, 146.1, 159.0, 159.6, 164.3; LC-MS (ESI) $m / z 339\left([\mathrm{M}+1]^{+}\right)$.

2-[4-(4-Cyanophenyl)-1H-1,2,3-triazol-1-yl]-N-(3-methoxyphenyl)acetamide (1cac). White solid; Yield: 87\%. ${ }^{1} \mathrm{H}-\mathrm{NMR}\left(\mathrm{DMSO}_{-} \mathrm{d}_{6}\right) \delta 3.72(\mathrm{~s}, 3 \mathrm{H}), 5.41(\mathrm{~s}, 2 \mathrm{H}), 6.68(\mathrm{dd}, J=1.9,8.3 \mathrm{~Hz}, 1 \mathrm{H}), 7.11(\mathrm{~d}$, $J=8.1 \mathrm{~Hz}, 1 \mathrm{H}), 7.25(\mathrm{t}, J=8.2 \mathrm{~Hz}, 1 \mathrm{H}), 7.29(\mathrm{t}, J=2.1 \mathrm{~Hz}, 1 \mathrm{H}), 7.93(\mathrm{~d}, J=8.6 \mathrm{~Hz}, 2 \mathrm{H}), 8.08(\mathrm{~d}$, $J=8.6 \mathrm{~Hz}, 2 \mathrm{H}), 8.81(\mathrm{~s}, 1 \mathrm{H}), 10.52(\mathrm{~s}, 1 \mathrm{H}) ;{ }^{13} \mathrm{C}-\mathrm{NMR}\left(\mathrm{DMSO}-d_{6}\right) \delta 52.5,55.0,105.0,109.3,110.1,111.5$, 124.7, 125.7, 129.7, 133.0, 135.2, 139.5, 144.6, 159.6, 164.0; LC-MS (ESI) $m / z 334\left([\mathrm{M}+1]^{+}\right)$.

N-(3-Methoxyphenyl)-2-[4-(thiophen-3-yl)-1H-1,2,3-triazol-1-yl]acetamide (1cad). Light brown solid; Yield: 76\%. ${ }^{1} \mathrm{H}-\mathrm{NMR}\left(\mathrm{DMSO}-\mathrm{d}_{6}\right) \delta 3.72(\mathrm{~s}, 3 \mathrm{H}), 5.36(\mathrm{~s}, 2 \mathrm{H}), 6.68(\mathrm{dd}, J=2.2,7.9 \mathrm{~Hz}, 1 \mathrm{H}), 7.11(\mathrm{dd}$, $J=0.9,8.1 \mathrm{~Hz}, 1 \mathrm{H}), 7.24(\mathrm{t}, J=8.2 \mathrm{~Hz}, 1 \mathrm{H}), 7.30(\mathrm{t}, J=2.1 \mathrm{~Hz}, 1 \mathrm{H}), 7.55(\mathrm{dd}, J=1.3,5.0 \mathrm{~Hz}, 1 \mathrm{H}), 7.66$ $(\mathrm{dd}, J=3.0,5.0 \mathrm{~Hz}, 1 \mathrm{H}), 7.88(\mathrm{dd}, J=1.2,3.0 \mathrm{~Hz}, 1 \mathrm{H}), 8.46(\mathrm{~s}, 1 \mathrm{H}), 10.50(\mathrm{~s}, 1 \mathrm{H}) ;{ }^{13} \mathrm{C}-\mathrm{NMR}$ (DMSO- $d_{6}$ ) $\delta 52.3,55.0,105.0,109.3,111.5,120.8,122.8,125.8,127.1,129.7,132.0,139.5,142.8,159.6,164.2$; LC-MS (ESI) $m / z 315\left([\mathrm{M}+1]^{+}\right)$.

N-(3-Methoxyphenyl)-2-[4-(pyridin-2-yl)-1H-1,2,3-triazol-1-yl]acetamide (1cae). Brown solid; Yield: 81\%. ${ }^{1} \mathrm{H}-\mathrm{NMR}\left(\mathrm{CDCl}_{3}\right) \delta 3.79(\mathrm{~s}, 3 \mathrm{H}), 5.26(\mathrm{~s}, 2 \mathrm{H}), 6.70(\mathrm{dd}, J=2.1,8.2 \mathrm{~Hz}, 1 \mathrm{H}), 6.95(\mathrm{~d}, J=8.0 \mathrm{~Hz}, 1 \mathrm{H})$, $7.18-7.22(\mathrm{~m}, 2 \mathrm{H}), 7.28(\mathrm{~m}, 1 \mathrm{H}), 7.82(\mathrm{t}, J=7.6 \mathrm{~Hz}, 1 \mathrm{H}), 7.87(\mathrm{~s}, 1 \mathrm{H}), 8.19(\mathrm{~d}, J=7.4 \mathrm{~Hz}, 1 \mathrm{H}), 8.33(\mathrm{~s}, 1 \mathrm{H})$, 8.61 (br s, $1 \mathrm{H}$ ); ${ }^{13} \mathrm{C}-\mathrm{NMR}$ (DMSO- $d_{6}$ ) $\delta$ 52.4, 55.1, 105.1, 109.4, 115.6, 119.5, 123.1, 125.1, 129.9, 137.4, 139.6, 147.1, 149.8, 150.0, 159.7, 164.3; LC-MS (ESI) $m / z 310\left([\mathrm{M}+1]^{+}\right)$.

$\mathrm{N}$-(3-Methoxyphenyl)-2-[4-(2-methoxyphenyl)-1H-1,2,3-triazol-1-yl]acetamide (1cah). Light yellow solid; Yield: 83\%. ${ }^{1} \mathrm{H}-\mathrm{NMR}\left(\mathrm{CDCl}_{3}\right) \delta 3.78(\mathrm{~s}, 3 \mathrm{H}), 3.96(\mathrm{~s}, 3 \mathrm{H}), 5.24(\mathrm{~s}, 2 \mathrm{H}), 6.96(\mathrm{dd}, J=1.3,8.1 \mathrm{~Hz}, 1 \mathrm{H}), 7.01$ $(\mathrm{d}, J=8.3 \mathrm{~Hz}, 1 \mathrm{H}), 7.11(\mathrm{dt}, J=0.9,7.6 \mathrm{~Hz}, 1 \mathrm{H}), 7.17(\mathrm{t}, J=2.2 \mathrm{~Hz}, 1 \mathrm{H}), 7.20(\mathrm{t}, J=8.2 \mathrm{~Hz}, 1 \mathrm{H}), 7.36(\mathrm{~m}$, 1H), $7.92(\mathrm{br} \mathrm{s}, 1 \mathrm{H}), 8.24(\mathrm{~s}, 1 \mathrm{H}), 8.36(\mathrm{dd}, J=1.7,7.8 \mathrm{~Hz}, 1 \mathrm{H}) ;{ }^{13} \mathrm{C}-\mathrm{NMR}\left(\mathrm{DMSO}-d_{6}\right) \delta 52.2,55.0,55.5$, 105.0, 109.2, 111.4, 111.5, 119.1, 120.6, 125.6, 126.5, 128.8, 129.7, 139.6, 141.6, 155.3, 159.5, 164.4; LC-MS (ESI) $m / z 339\left([\mathrm{M}+1]^{+}\right)$.

N-(3-Methoxyphenyl)-2-(4-m-tolyl-1H-1,2,3-triazol-1-yl)acetamide (1caj). White solid; Yield: 93\%. ${ }^{1} \mathrm{H}-\mathrm{NMR}\left(\mathrm{CDCl}_{3}\right) \delta 2.42(\mathrm{~s}, 3 \mathrm{H}), 3.79(\mathrm{~s}, 3 \mathrm{H}), 5.23(\mathrm{~s}, 2 \mathrm{H}), 6.70(\mathrm{dd}, J=2.2,8.1 \mathrm{~Hz}, 1 \mathrm{H}), 6.97(\mathrm{dd}$, $J=1.3,8.0 \mathrm{~Hz}, 1 \mathrm{H}), 7.19-7.23(\mathrm{~m}, 3 \mathrm{H}), 7.34(\mathrm{t}, J=7.7 \mathrm{~Hz}, 1 \mathrm{H}), 7.63(\mathrm{~d}, J=7.6 \mathrm{~Hz}, 1 \mathrm{H}), 7.71(\mathrm{~s}, 1 \mathrm{H}), 7.94$ (br s, $1 \mathrm{H}), 7.96(\mathrm{~s}, 1 \mathrm{H}) ;{ }^{13} \mathrm{C}-\mathrm{NMR}$ (DMSO- $\left.d_{6}\right) \delta 52.4,55.0,55.1,105.0,109.3,110.3,111.5,113.6,117.5$, 123.7, 129.7, 130.0, 132.0, 139.5, 146.1, 159.6, 159.7, 164.2; LC-MS (ESI) $m / z 323\left([\mathrm{M}+1]^{+}\right)$.

N-(3-Methoxyphenyl)-2-(4-o-tolyl-1H-1,2,3-triazol-1-yl)acetamide (1cak). Light yellow solid; Yield: 90\%. ${ }^{1} \mathrm{H}-\mathrm{NMR}\left(\mathrm{CDCl}_{3}\right) \delta 2.50(\mathrm{~s}, 3 \mathrm{H}), 3.79(\mathrm{~s}, 3 \mathrm{H}), 5.26(\mathrm{~s}, 2 \mathrm{H}), 6.71(\mathrm{dd}, J=2.1,8.3 \mathrm{~Hz}, 1 \mathrm{H}), 6.98(\mathrm{dd}, J=1.3$, $8.0 \mathrm{~Hz}, 1 \mathrm{H}), 7.19-7.24(\mathrm{~m}, 2 \mathrm{H}), 7.28-7.32(\mathrm{~m}, 3 \mathrm{H}), 7.79(\mathrm{~m}, 1 \mathrm{H}), 7.87(\mathrm{~s}, 1 \mathrm{H}), 8.00(\mathrm{br} \mathrm{s}, 1 \mathrm{H}) ;{ }^{13} \mathrm{C}-\mathrm{NMR}$ (DMSO-d $d_{6}$ ) $\delta$ 21.1, 52.3, 55.0, 105.0, 109.3, 111.5, 124.9, 126.1, 127.8, 128.1, 129.7, 123.0, 130.9, 134.8, 139.6, 145.4, 159.6, 164.3; LC-MS (ESI) $m / z 323\left([\mathrm{M}+1]^{+}\right)$.

2-\{4-[4-(Dimethylamino)phenyl]-1H-1,2,3-triazol-1-yl\}-N-(3-methoxyphenyl)acetamide (1cal). Brown solid; Yield: $91 \% .{ }^{1} \mathrm{H}-\mathrm{NMR}\left(\mathrm{DMSO}_{-} d_{6}\right) \delta 2.94(\mathrm{~s}, 6 \mathrm{H}), 3.72(\mathrm{~s}, 3 \mathrm{H}), 5.32(\mathrm{~s}, 2 \mathrm{H}), 6.68(\mathrm{dd}, J=1.9,8.3 \mathrm{~Hz}, 1 \mathrm{H})$, $6.79(\mathrm{~d}, J=9.0 \mathrm{~Hz}, 2 \mathrm{H}), 7.11(\mathrm{dd}, J=1.0,8.1 \mathrm{~Hz}, 1 \mathrm{H}), 7.24(\mathrm{t}, J=8.2 \mathrm{~Hz}, 1 \mathrm{H}), 7.30(\mathrm{t}, J=2.1 \mathrm{~Hz}, 1 \mathrm{H})$, $7.67(\mathrm{~d}, J=8.9 \mathrm{~Hz}, 2 \mathrm{H}), 8.36(\mathrm{~s}, 1 \mathrm{H}), 10.48(\mathrm{~s}, 1 \mathrm{H}) ;{ }^{13} \mathrm{C}-\mathrm{NMR}\left(\mathrm{DMSO}-d_{6}\right) \delta 40.0,52.3,55.0,105.0,109.3$, 111.5, 112.4 $118.6,121.2,126.1,129.7,139.6,146.8,150.1,159.6,164.3$; LC-MS (ESI) $\mathrm{m} / z 352\left([\mathrm{M}+1]^{+}\right)$.

N-(3-Methoxyphenyl)-2-[4-(4-phenoxyphenyl)-1H-1,2,3-triazol-1-yl]acetamide (1cam). Yellow solid; Yield: 91\%. ${ }^{1} \mathrm{H}-\mathrm{NMR}\left(\mathrm{DMSO}-d_{6}\right) \delta 3.72(\mathrm{~s}, 3 \mathrm{H}), 5.37(\mathrm{~s}, 2 \mathrm{H}), 6.68(\mathrm{dd}, J=1.8,8.3 \mathrm{~Hz}, 1 \mathrm{H}), 7.06-7.10(\mathrm{~m}, 4 \mathrm{H})$, $7.12(\mathrm{dd}, J=1.0,8.1 \mathrm{~Hz}, 1 \mathrm{H}), 7.17(\mathrm{~m}, 1 \mathrm{H}), 7.25(\mathrm{t}, J=8.2 \mathrm{~Hz}, 1 \mathrm{H}), 7.30(\mathrm{t}, J=2.2 \mathrm{~Hz}, 1 \mathrm{H}), 7.40-7.44(\mathrm{~m}$, 2H), $7.88(\mathrm{~d}, J=8.8 \mathrm{~Hz}, 2 \mathrm{H}), 8.55(\mathrm{~s}, 1 \mathrm{H}), 10.50(\mathrm{~s}, 1 \mathrm{H}) ;{ }^{13} \mathrm{C}-\mathrm{NMR}\left(\right.$ DMSO- $\left._{6}\right) \delta 52.4,55.0,105.0,109.3$, 111.5, 118.8, 118.9, 122.7, 123.7, 126.0, 126.9, 129.7, 130.1, 139.5, 145.7, 156.4, 159.6, 164.2; LC-MS (ESI) $m / z 401\left([\mathrm{M}+1]^{+}\right)$. 
2-[4-(2,4-Difluorophenyl)-1H-1,2,3-triazol-1-yl]-N-(3-methoxyphenyl)acetamide (1can). Light yellow solid; Yield: 92\%. ${ }^{1} \mathrm{H}-\mathrm{NMR}\left(\mathrm{CDCl}_{3}\right) \delta 3.79(\mathrm{~s}, 3 \mathrm{H}), 5.25(\mathrm{~s}, 2 \mathrm{H}), 6.70(\mathrm{dd}, J=2.0,8.4 \mathrm{~Hz}, 1 \mathrm{H}), 6.93(\mathrm{ddd}$, $J=2.5,8.7,11.1 \mathrm{~Hz}, 1 \mathrm{H}), 6.95(\mathrm{dd}, J=1.7,7.7 \mathrm{~Hz}, 1 \mathrm{H}), 7.03(\mathrm{~m}, 1 \mathrm{H}), 7.19(\mathrm{t}, J=2.2 \mathrm{~Hz}, 1 \mathrm{H}), 7.22(\mathrm{t}$, $J=8.2 \mathrm{~Hz}, 1 \mathrm{H}), 7.81(\mathrm{br} \mathrm{s}, 1 \mathrm{H}), 8.10(\mathrm{~d}, J=3.5 \mathrm{~Hz}, 1 \mathrm{H}), 8.30(\mathrm{dt}, J=6.5,8.6 \mathrm{~Hz}, 1 \mathrm{H}) ;{ }^{13} \mathrm{C}-\mathrm{NMR}$ $\left(\right.$ DMSO- $\left.d_{6}\right) \delta 52.3,55.0,104.6\left(\mathrm{t}, J_{\mathrm{CF}}=26.1 \mathrm{~Hz}\right), 105.0,109.27,111.4,112.3\left(\mathrm{dd}, J_{\mathrm{CF}}=21.4,3.5 \mathrm{~Hz}\right)$, $115.2\left(\mathrm{dd}, J_{\mathrm{CF}}=13.4,3.7 \mathrm{~Hz}\right), 125.2\left(\mathrm{~d}, J_{\mathrm{CF}}=10.9 \mathrm{~Hz}\right), 128.5\left(\mathrm{dd}, J_{\mathrm{CF}}=9.8,5.3 \mathrm{~Hz}\right), 129.7,138.9(\mathrm{~d}$, $\left.J_{\mathrm{CF}}=2.6 \mathrm{~Hz}\right), 139.6,158.5\left(\mathrm{dd}, J_{\mathrm{CF}}=250.7,12.8\right), 159.6,161.7\left(\mathrm{dd}, J_{\mathrm{CF}}=247.5,12.5 \mathrm{~Hz}\right), 164.2 ; \mathrm{LC}-\mathrm{MS}$ (ESI) $m / z 345\left([\mathrm{M}+1]^{+}\right)$.

2-(4-Benzyl-1H-1,2,3-triazol-1-yl)-N-(3-methoxyphenyl)acetamide (1cao). White solid; Yield: 88\%. ${ }^{1} \mathrm{H}-\mathrm{NMR}\left(\mathrm{CDCl}_{3}\right) \delta 3.77(\mathrm{~s}, 3 \mathrm{H}), 4.12(\mathrm{~s}, 2 \mathrm{H}), 5.10(\mathrm{~s}, 2 \mathrm{H}), 6.68(\mathrm{dd}, J=2.1,8.2 \mathrm{~Hz}, 1 \mathrm{H}), 6.94(\mathrm{dd}$, $J=1.1,7.9 \mathrm{~Hz}, 1 \mathrm{H}), 7.17-7.32(\mathrm{~m}, 7 \mathrm{H}), 7.39(\mathrm{~s}, 1 \mathrm{H}), 8.15(\mathrm{br} \mathrm{s}, 1 \mathrm{H}) ;{ }^{13} \mathrm{C}-\mathrm{NMR}$ (DMSO- $\left.d_{6}\right) \delta 31.2,52.1$, 55.0, 105.0, 109.2, 111.4, 124.1, 126.1, 128.4, 128.5, 129.7, 139.56, 139.59, 145.9, 159.5, 164.3; LC-MS (ESI) $\mathrm{m} / \mathrm{z} 323\left([\mathrm{M}+1]^{+}\right)$.

N-(2-Methoxyphenyl)-2-(4-phenyl-1H-1,2,3-triazol-1-yl)acetamide (1daa). Light yellow solid; Yield: 94\%. ${ }^{1} \mathrm{H}-\mathrm{NMR}\left(\mathrm{CDCl}_{3}\right) \delta 3.80(\mathrm{~s}, 3 \mathrm{H}), 5.27(\mathrm{~s}, 2 \mathrm{H}), 6.85(\mathrm{~d}, J=8.2 \mathrm{~Hz}, 1 \mathrm{H}), 6.96(\mathrm{dt}, J=1.1,7.8 \mathrm{~Hz}, 1 \mathrm{H}), 7.08$ $(\mathrm{dt}, J=1.4,7.9 \mathrm{~Hz}, 1 \mathrm{H}), 7.37(\mathrm{~m}, 1 \mathrm{H}), 7.44-7.47(\mathrm{~m}, 2 \mathrm{H}), 7.86-7.88(\mathrm{~m}, 2 \mathrm{H}), 7.99(\mathrm{~s}, 1 \mathrm{H}), 8.24(\mathrm{~s}, 1 \mathrm{H})$, $8.27(\mathrm{dd}, J=1.4,8.1 \mathrm{~Hz}, 1 \mathrm{H}) ;{ }^{13} \mathrm{C}-\mathrm{NMR}\left(\mathrm{DMSO}_{6}{ }_{6}\right) \delta 52.3,55.7,111.3,120.3,121.7,123.0,124.9,125.1$, $126.6,127.8,128.9,130.7,146.2,149.6,164.4$; LC-MS (ESI) $m / z 309\left([\mathrm{M}+1]^{+}\right)$.

N-(2-Methoxyphenyl)-2-[4-(4-methoxyphenyl)-1H-1,2,3-triazol-1-yl]acetamide (1dab). White solid; Yield: 90\%. ${ }^{1} \mathrm{H}-\mathrm{NMR}\left(\mathrm{CDCl}_{3}\right) \delta 3.79(\mathrm{~s}, 3 \mathrm{H}), 3.85(\mathrm{~s}, 3 \mathrm{H}), 5.25(\mathrm{~s}, 2 \mathrm{H}), 6.84(\mathrm{dd}, J=1.0,8.2 \mathrm{~Hz}, 1 \mathrm{H}), 6.95$ $(\mathrm{dt}, J=1.1,7.7 \mathrm{~Hz}, 1 \mathrm{H}), 6.98(\mathrm{~d}, J=8.9 \mathrm{~Hz}, 2 \mathrm{H}), 7.07(\mathrm{dt}, J=1.5,7.9 \mathrm{~Hz}, 1 \mathrm{H}), 7.79(\mathrm{~d}, J=8.9 \mathrm{~Hz}$, 2H), $7.90(\mathrm{~s}, 1 \mathrm{H}), 8.24(\mathrm{~s}, 1 \mathrm{H}), 8.27(\mathrm{dd}, J=1.5,8.1 \mathrm{~Hz}, 1 \mathrm{H}) ;{ }^{13} \mathrm{C}-\mathrm{NMR}\left(\mathrm{DMSO}-d_{6}\right) \delta 52.3,55.1,55.7$, $111.3,114.3,120.3,121.7,122.1,123.3,124.9,126.5,126.6,146.1,149.6,159.0,164.5$; LC-MS (ESI) $\mathrm{m} / z 339$ $\left([\mathrm{M}+1]^{+}\right)$.

2-[4-(4-Cyanophenyl)-1H-1,2,3-triazol-1-yl]-N-(2-methoxyphenyl)acetamide (1dac). White solid; Yield: 86\%. ${ }^{1} \mathrm{H}-\mathrm{NMR}\left(\right.$ DMSO- $\left.d_{6}\right) \delta 3.88(\mathrm{~s}, 3 \mathrm{H}), 5.52(\mathrm{~s}, 2 \mathrm{H}), 6.91(\mathrm{~m}, 1 \mathrm{H}), 7.08-7.13(\mathrm{~m}, 2 \mathrm{H}), 7.92-7.94(\mathrm{~m}$, $3 \mathrm{H}), 8.08(\mathrm{~d}, J=8.4 \mathrm{~Hz}, 2 \mathrm{H}), 8.80(\mathrm{~s}, 1 \mathrm{H}), 9.81(\mathrm{~s}, 1 \mathrm{H}) ;{ }^{13} \mathrm{C}-\mathrm{NMR}\left(\mathrm{DMSO}-d_{6}\right) \delta 52.4,55.7,110.1,111.3$, $120.3,121.8,124.8,125.0,125.7,126.5,132.6,132.6,133.0,135.2,144.6,149.6,164.3$; LC-MS (ESI) $\mathrm{m} / \mathrm{z} 334$ $\left([\mathrm{M}+1]^{+}\right)$.

N-(2-Methoxyphenyl)-2-[4-(thiophen-3-yl)-1H-1,2,3-triazol-1-yl]acetamide (1dad). Light brown solid; Yield: 91\%. ${ }^{1} \mathrm{H}-\mathrm{NMR}\left(\mathrm{CDCl}_{3}\right) \delta 3.81(\mathrm{~s}, 3 \mathrm{H}), 5.25(\mathrm{~s}, 2 \mathrm{H}), 6.85(\mathrm{dd}, J=1.0,8.2 \mathrm{~Hz}, 1 \mathrm{H}), 6.96(\mathrm{dt}$, $J=1.1,7.9 \mathrm{~Hz}, 1 \mathrm{H}), 7.08(\mathrm{dt}, J=1.4,7.9 \mathrm{~Hz}, 1 \mathrm{H}), 7.41(\mathrm{dd}, J=3.0,5.0 \mathrm{~Hz}, 1 \mathrm{H}), 7.49(\mathrm{dd}, J=1.2,5.1 \mathrm{~Hz}$, $1 \mathrm{H}), 7.74(\mathrm{dd}, J=1.2,3.0 \mathrm{~Hz}, 1 \mathrm{H}), 7.89(\mathrm{~s}, 1 \mathrm{H}), 8.22(\mathrm{br} \mathrm{s}, 1 \mathrm{H}), 8.27(\mathrm{dd}, J=1.5,8.1 \mathrm{~Hz}, 1 \mathrm{H}) ;{ }^{13} \mathrm{C}-\mathrm{NMR}$ (DMSO- $d_{6}$ ) $\delta 52.3,55.7,111.3,120.3,120.8,121.7,122.8,124.9,125.8,126.6,127.1,132.0,142.7,149.6$, 164.4; LC-MS (ESI) $m / z 315\left([\mathrm{M}+1]^{+}\right)$.

N-(2-Methoxyphenyl)-2-[4-(pyridin-2-yl)-1H-1,2,3-triazol-1-yl]acetamide (1dae). Light brown solid; Yield: $84 \% .{ }^{1} \mathrm{H}-\mathrm{NMR}\left(\mathrm{CDCl}_{3}\right) \delta 3.80(\mathrm{~s}, 3 \mathrm{H}), 5.28(\mathrm{~s}, 2 \mathrm{H}), 6.85(\mathrm{~d}, J=8.2 \mathrm{~Hz}, 1 \mathrm{H}), 6.95(\mathrm{~m}, 1 \mathrm{H}), 7.07(\mathrm{dt}$, $J=1.4,7.9 \mathrm{~Hz}, 1 \mathrm{H}), 7.27(\mathrm{~m}, 1 \mathrm{H}), 7.80(\mathrm{dt}, J=1.7,7.8 \mathrm{~Hz}, 1 \mathrm{H}), 8.20(\mathrm{~d}, J=7.9 \mathrm{~Hz}, 1 \mathrm{H}), 8.25(\mathrm{br} \mathrm{s}, 1 \mathrm{H})$, $8.27(\mathrm{dd}, J=1.4,8.1 \mathrm{~Hz}, 1 \mathrm{H}), 8.34(\mathrm{~s}, 1 \mathrm{H}), 8.61(\mathrm{~d}, J=4.2 \mathrm{~Hz}, 1 \mathrm{H}) ;{ }^{13} \mathrm{C}-\mathrm{NMR}\left(\mathrm{DMSO}-d_{6}\right) \delta 52.4,55.8$, 111.4, 119.5, 120.4, 121.9, 123.1, 125.06., 125.10, 126.6, 137.3, 147.1, 149.7, 150.1, 164.5; LC-MS (ESI) $\mathrm{m} / \mathrm{z}$ $310\left([\mathrm{M}+1]^{+}\right)$.

N-(2-Methoxyphenyl)-2-[4-(3-methoxyphenyl)-1H-1,2,3-triazol-1-yl]acetamide (1dag). White solid; Yield: $86 \% .{ }^{1} \mathrm{H}-\mathrm{NMR}\left(\mathrm{CDCl}_{3}\right) \delta 3.81(\mathrm{~s}, 3 \mathrm{H}), 3.88(\mathrm{~s}, 3 \mathrm{H}), 5.26(\mathrm{~s}, 2 \mathrm{H}), 6.85(\mathrm{~d}, J=8.2 \mathrm{~Hz}, 1 \mathrm{H}), 6.92(\mathrm{ddd}$, $J=1.0,2.6,8.1 \mathrm{~Hz}, 1 \mathrm{H}), 6.96(\mathrm{dt}, J=1.0,7.8 \mathrm{~Hz}, 1 \mathrm{H}), 7.08(\mathrm{dt}, J=1.4,7.9 \mathrm{~Hz}, 1 \mathrm{H}), 7.35(\mathrm{t}, J=7.9 \mathrm{~Hz}$, $1 \mathrm{H}), 7.40(\mathrm{td}, J=1.2,7.6 \mathrm{~Hz}, 1 \mathrm{H}), 7.48(\mathrm{dd}, J=1.5,2.4 \mathrm{~Hz}, 1 \mathrm{H}), 7.98(\mathrm{~s}, 1 \mathrm{H}), 8.23(\mathrm{br} \mathrm{s}, 1 \mathrm{H}), 8.27(\mathrm{dd}$, $J=1.3,8.1 \mathrm{~Hz}, 1 \mathrm{H}$ ); ${ }^{13} \mathrm{C}-\mathrm{NMR}$ (DMSO- $d_{6}$ ) $\delta 52.3,55.1,55.7,110.3,111.3,113.6,117.5,120.3,121.8,123.3$, $124.9,126.6,130.1,132.1,146.1,149.6,159.7,164.4$; LC-MS (ESI) $\mathrm{m} / z 339\left([\mathrm{M}+1]^{+}\right)$. 
N-(2-Methoxyphenyl)-2-[4-(2-methoxyphenyl)-1H-1,2,3-triazol-1-yl]acetamide (1dah). Light yellow solid; Yield: 80\%. ${ }^{1} \mathrm{H}-\mathrm{NMR}\left(\mathrm{CDCl}_{3}\right) \delta 3.77(\mathrm{~s}, 3 \mathrm{H}), 3.95(\mathrm{~s}, 3 \mathrm{H}), 5.26(\mathrm{~s}, 2 \mathrm{H}), 6.83(\mathrm{dd}, J=1.1,8.2 \mathrm{~Hz}, 1 \mathrm{H})$, $6.95(\mathrm{dt}, J=1.2,7.8 \mathrm{~Hz}, 1 \mathrm{H}), 7.00(\mathrm{dd}, J=0.6,8.3 \mathrm{~Hz}, 1 \mathrm{H}), 7.06(\mathrm{dt}, J=1.5,7.9 \mathrm{~Hz}, 1 \mathrm{H}), 7.11(\mathrm{dt}$, $J=1.0,7.6 \mathrm{~Hz}, 1 \mathrm{H}), 7.35(\mathrm{ddd}, J=1.7,7.4,8.3 \mathrm{~Hz}, 1 \mathrm{H}), 8.25(\mathrm{~s}, 1 \mathrm{H}), 8.27$ (br s, 1H), $8.28(\mathrm{dd}$, $J=1.5,8.1 \mathrm{~Hz}, 1 \mathrm{H}), 8.39(\mathrm{dd}, J=1.7,7.7 \mathrm{~Hz}, 1 \mathrm{H}) ;{ }^{13} \mathrm{C}-\mathrm{NMR}\left(\mathrm{DMSO}-d_{6}\right) \delta 52.2,55.5,55.7,111.3$, $111.5,119.1,120.3,120.7,121.7,124.9,125.6,126.5,126.6,128.9,141.6,149.6,155.3,164.6$; LC-MS (ESI) $m / z 339\left([\mathrm{M}+1]^{+}\right)$.

N-(2-Methoxyphenyl)-2-(4-p-tolyl-1H-1,2,3-triazol-1-yl)acetamide (1dai). Light yellow solid; Yield: 78\%. ${ }^{1} \mathrm{H}-\mathrm{NMR}\left(\mathrm{CDCl}_{3}\right) \delta 2.39(\mathrm{~s}, 3 \mathrm{H}), 3.80(\mathrm{~s}, 3 \mathrm{H}), 5.25(\mathrm{~s}, 2 \mathrm{H}), 6.85(\mathrm{~d}, J=8.2 \mathrm{~Hz}, 1 \mathrm{H}), 6.96(\mathrm{t}, J=7.8 \mathrm{~Hz}$, $1 \mathrm{H}), 7.07(\mathrm{dt}, J=1.4,7.9 \mathrm{~Hz}, 1 \mathrm{H}), 7.26(\mathrm{~d}, J=7.8 \mathrm{~Hz}, 2 \mathrm{H}), 7.75(\mathrm{~d}, J=8.2 \mathrm{~Hz}, 2 \mathrm{H}), 7.94(\mathrm{~s}, 1 \mathrm{H}), 8.23(\mathrm{br}$ $\mathrm{s}, 1 \mathrm{H}), 8.27(\mathrm{dd}, J=1.5,8.0 \mathrm{~Hz}, 1 \mathrm{H}) ;{ }^{13} \mathrm{C}-\mathrm{NMR}\left(\mathrm{DMSO}-d_{6}\right) \delta 20.8,52.3,55.7,111.3,120.3,121.7,122.6$, $124.9,125.1,126.6,128.0,129.5,137.1,146.2,149.6,164.5$; LC-MS (ESI) $m / z 323\left([\mathrm{M}+1]^{+}\right)$.

N-(2-Methoxyphenyl)-2-(4-m-tolyl-1H-1,2,3-triazol-1-yl)acetamide (1daj). Light yellow solid; Yield: 85\%. ${ }^{1} \mathrm{H}-\mathrm{NMR}\left(\mathrm{CDCl}_{3}\right) \delta 2.42(\mathrm{~s}, 3 \mathrm{H}), 3.80(\mathrm{~s}, 3 \mathrm{H}), 5.26(\mathrm{~s}, 2 \mathrm{H}), 6.85(\mathrm{dd}, J=0.9,8.2 \mathrm{~Hz}, 1 \mathrm{H}), 6.95(\mathrm{dt}$, $J=1.0,7.8 \mathrm{~Hz}, 1 \mathrm{H}), 7.08(\mathrm{dt}, J=1.4,7.8 \mathrm{~Hz}, 1 \mathrm{H}), 7.18(\mathrm{~d}, J=7.6 \mathrm{~Hz}, 1 \mathrm{H}), 7.33(\mathrm{t}, J=7.6 \mathrm{~Hz}, 1 \mathrm{H}), 7.64(\mathrm{~d}$, $J=7.7 \mathrm{~Hz}, 1 \mathrm{H}), 7.72(\mathrm{~s}, 1 \mathrm{H}), 7.97(\mathrm{~s}, 1 \mathrm{H}), 8.25$ (br s, 1H), 8.27 (dd, $J=1.4,8.1 \mathrm{~Hz}, 1 \mathrm{H}) ;{ }^{13} \mathrm{C}-\mathrm{NMR}$ $\left(\mathrm{DMSO}-d_{6}\right) \delta 21.1,52.3,55.7,111.3,120.3,121.7,122.3,123.0,124.9,125.7,126.6,128.5,128.8,130.6$, 138.1, 146.3, 149.6, 164.4; LC-MS (ESI) $m / z 323\left([\mathrm{M}+1]^{+}\right)$.

2-\{4-[4-(Dimethylamino)phenyl]-1H-1,2,3-triazol-1-yl\}-N-(2-methoxyphenyl)acetamide (1dal). Brown solid; Yield: 88\%. ${ }^{1} \mathrm{H}-\mathrm{NMR}\left(\mathrm{CDCl}_{3}\right) \delta 3.00(\mathrm{~s}, 6 \mathrm{H}), 3.78(\mathrm{~s}, 3 \mathrm{H}), 5.23(\mathrm{~s}, 2 \mathrm{H}), 6.78(\mathrm{~d}, J=8.9 \mathrm{~Hz}, 2 \mathrm{H}), 6.84(\mathrm{dd}$, $J=1.0,8.1 \mathrm{~Hz}, 1 \mathrm{H}), 6.95(\mathrm{dt}, J=1.1,7.8 \mathrm{~Hz}, 1 \mathrm{H}), 7.07(\mathrm{dt}, J=1.4,7.8 \mathrm{~Hz}, 1 \mathrm{H}), 7.73(\mathrm{~d}, J=9.0 \mathrm{~Hz}, 2 \mathrm{H})$, 7.83 (s, $1 \mathrm{H}), 8.24$ (br s, $1 \mathrm{H}), 8.27$ (dd, $J=1.5,8.1 \mathrm{~Hz}, 1 \mathrm{H}) ;{ }^{13} \mathrm{C}-\mathrm{NMR}\left(\mathrm{DMSO}-d_{6}\right) \delta 40.0,52.3,55.7,111.3$, $112.4,118.6,120.3,121.1,121.7,124.9,126.0,146.8,149.6,150.0,164.5 ;$ LC-MS (ESI) $m / z 352\left([\mathrm{M}+1]^{+}\right)$.

N-(2-Methoxyphenyl)-2-[4-(4-phenoxyphenyl)-1H-1,2,3-triazol-1-yl]acetamide (1dam). Yellow solid; Yield: 92\%. ${ }^{1} \mathrm{H}-\mathrm{NMR}\left(\mathrm{CDCl}_{3}\right) \delta 3.81(\mathrm{~s}, 3 \mathrm{H}), 5.26(\mathrm{~s}, 2 \mathrm{H}), 6.86(\mathrm{dd}, J=1.0,8.2 \mathrm{~Hz}, 1 \mathrm{H}), 6.96(\mathrm{dt}, J=1.1,7.8 \mathrm{~Hz}$, $1 \mathrm{H}), 7.02-7.09(\mathrm{~m}, 5 \mathrm{H}), 7.14(\mathrm{~m}, 1 \mathrm{H}), 7.35-7.38(\mathrm{~m}, 2 \mathrm{H}), 7.83(\mathrm{~d}, J=8.8 \mathrm{~Hz}, 2 \mathrm{H}), 7.94(\mathrm{~s}, 1 \mathrm{H}), 8.24$ (br s, 1H), 8.27 (dd, $J=1.5,8.1 \mathrm{~Hz}, 1 \mathrm{H}) ;{ }^{13} \mathrm{C}-\mathrm{NMR}\left(\mathrm{DMSO}-d_{6}\right) \delta 52.3,55.7,111.3,118.8,120.3,121.7$, 122.7, 123.6, 124.9, 126.1, 126.6, 126.9, 130.1, 145.7, 149.6, 156.40, 156.41, 166.4; LC-MS (ESI) $\mathrm{m} / z 401$ $\left([\mathrm{M}+1]^{+}\right)$.

2-[4-(2,4-Difluorophenyl)-1H-1,2,3-triazol-1-yl]-N-(2-methoxyphenyl)acetamide (1dan). Light yellow solid; Yield: 94\%. ${ }^{1} \mathrm{H}-\mathrm{NMR}\left(\mathrm{CDCl}_{3}\right) \delta 3.82(\mathrm{~s}, 3 \mathrm{H}), 5.28(\mathrm{~s}, 2 \mathrm{H}), 6.86(\mathrm{dd}, J=1.0,8.2 \mathrm{~Hz}, 1 \mathrm{H}), 6.93$ $(\mathrm{ddd}, J=2.5,8.6,11.0 \mathrm{~Hz}, 1 \mathrm{H}), 6.96(\mathrm{dt}, J=1.1,7.8 \mathrm{~Hz}, 1 \mathrm{H}), 7.03(\mathrm{~m}, 1 \mathrm{H}), 7.08(\mathrm{dt}, J=1.4,7.9 \mathrm{~Hz}, 1 \mathrm{H})$, $8.12(\mathrm{~d}, J=3.6 \mathrm{~Hz}, 1 \mathrm{H}), 8.21(\mathrm{br} \mathrm{s}, 1 \mathrm{H}), 8.27(\mathrm{dd}, J=1.4,8.1 \mathrm{~Hz}, 1 \mathrm{H}), 8.31(\mathrm{dt}, J=6.5,8.6 \mathrm{~Hz}, 1 \mathrm{H})$; ${ }^{13}$ C-NMR (DMSO- $\left.d_{6}\right) \delta 52.3,55.7,104.6(t, J=26.0 \mathrm{~Hz}), 111.3,112.3(\mathrm{dd}, J=21.4,3.5 \mathrm{~Hz}), 115.2(\mathrm{dd}$, $J=13.5,3.7 \mathrm{~Hz}), 120.3,121.8,124.9,125.2(\mathrm{~d}, J=11.1 \mathrm{~Hz}), 126.6,128.5(\mathrm{dd}, J=9.7,5.3 \mathrm{~Hz}), 138.8(\mathrm{~d}$, $J=2.9 \mathrm{~Hz}), 149.6,158.5\left(\mathrm{dd}, J_{\mathrm{CF}}=250.5,12.5 \mathrm{~Hz}\right), 161.7\left(\mathrm{dd}, J_{\mathrm{CF}}=247.4,12.5 \mathrm{~Hz}\right), 164.4 ; \mathrm{LC}-\mathrm{MS}(\mathrm{ESI})$ $m / z 345\left([\mathrm{M}+1]^{+}\right)$.

2-(4-Benzyl-1H-1,2,3-triazol-1-yl)-N-(2-methoxyphenyl)acetamide (1dao). White solid; Yield: 88\%. ${ }^{1} \mathrm{H}-\mathrm{NMR}\left(\mathrm{CDCl}_{3}\right) \delta 3.77(\mathrm{~s}, 3 \mathrm{H}), 4.14(\mathrm{~s}, 2 \mathrm{H}), 5.14(\mathrm{~s}, 2 \mathrm{H}), 6.84(\mathrm{dd}, J=0.9,8.2 \mathrm{~Hz}, 1 \mathrm{H}), 6.94(\mathrm{dt}$, $J=1.1,7.8 \mathrm{~Hz}, 1 \mathrm{H}), 7.07(\mathrm{dt}, J=1.4,7.9 \mathrm{~Hz}, 1 \mathrm{H}), 7.20-7.32(\mathrm{~m}, 5 \mathrm{H}), 7.40(\mathrm{~s}, 1 \mathrm{H}), 8.20(\mathrm{br} \mathrm{s}, 1 \mathrm{H}), 8.25$ $(\mathrm{dd}, J=1.4,8.0 \mathrm{~Hz}, 1 \mathrm{H}) ;{ }^{13} \mathrm{C}-\mathrm{NMR}\left(\mathrm{DMSO}_{-}\right)_{6} \delta 31.2,52.1,55.7,111.3,120.3,121.6,124.1,124.8,126.1$, $126.5,128.4,128.5,139.6,145.8,149.5,164.5$; LC-MS (ESI) $m / z 323\left([\mathrm{M}+1]^{+}\right)$.

N-Butyl-2-(4-phenyl-1H-1,2,3-triazol-1-yl)acetamide (1eaa). White solid; Yield: 81\%. ${ }^{1} \mathrm{H}-\mathrm{NMR}$ $\left(\mathrm{DMSO}-d_{6}\right) \delta 0.88(\mathrm{t}, J=7.3 \mathrm{~Hz}, 3 \mathrm{H}), 1.31(\mathrm{~m}, 2 \mathrm{H}), 1.43(\mathrm{~m}, 2 \mathrm{H}), 3.12(\mathrm{~m}, 2 \mathrm{H}), 5.11(\mathrm{~s}, 2 \mathrm{H}), 7.33(\mathrm{t}$, $J=7.4 \mathrm{~Hz}, 1 \mathrm{H}), 7.45(\mathrm{t}, J=7.7 \mathrm{~Hz}, 2 \mathrm{H}), 7.86(\mathrm{~d}, J=7.5 \mathrm{~Hz}, 2 \mathrm{H}), 8.32(\mathrm{br} \mathrm{s}, 1 \mathrm{H}), 8.51(\mathrm{~s}, 1 \mathrm{H}) ;{ }^{13} \mathrm{C}-\mathrm{NMR}$ $\left(\mathrm{DMSO}_{6}\right) \delta 13.6,19.5,31.0,38.4,51.8,122.9,125.1,127.8,128.9,130.7,146.1,165.1$; LC-MS (ESI) $\mathrm{m} / \mathrm{z}$ $259\left([\mathrm{M}+1]^{+}\right)$. 
N-Butyl-2-[4-(4-methoxyphenyl)-1H-1,2,3-triazol-1-yl]acetamide (1eab). White solid; Yield: $88 \%$. ${ }^{1} \mathrm{H}-\mathrm{NMR}\left(\mathrm{DMSO}-d_{6}\right) \delta 0.88(\mathrm{t}, J=7.3 \mathrm{~Hz}, 3 \mathrm{H}), 1.31(\mathrm{~m}, 2 \mathrm{H}), 1.43(\mathrm{~m}, 2 \mathrm{H}), 3.11(\mathrm{~m}, 2 \mathrm{H}), 3.79(\mathrm{~s}, 3 \mathrm{H})$, $5.09(\mathrm{~s}, 2 \mathrm{H}), 7.01(\mathrm{~d}, J=8.8 \mathrm{~Hz}, 2 \mathrm{H}), 7.78(\mathrm{~d}, J=8.8 \mathrm{~Hz}, 2 \mathrm{H}), 8.31$ (br s, $1 \mathrm{H}), 8.39(\mathrm{~s}, 1 \mathrm{H}) ;{ }^{13} \mathrm{C}-\mathrm{NMR}$ $\left(\mathrm{DMSO}-d_{6}\right) \delta 13.6,19.5,31.0,38.4,51.7,55.1,114.3,121.9,123.4,126.4,146.0,158.9,165.2$; LC-MS (ESI) $m / z 289\left([\mathrm{M}+1]^{+}\right)$.

N-Butyl-2-[4-(4-cyanophenyl)-1H-1,2,3-triazol-1-yl]acetamide (1eac). White solid; Yield: 83\%. ${ }^{1} \mathrm{H}-\mathrm{NMR}$ $\left(\mathrm{DMSO}_{-} \mathrm{d}_{6}\right) \delta 0.88(\mathrm{t}, J=7.3 \mathrm{~Hz}, 3 \mathrm{H}), 1.31(\mathrm{~m}, 2 \mathrm{H}), 1.43(\mathrm{~m}, 2 \mathrm{H}), 3.09(\mathrm{~m}, 2 \mathrm{H}), 5.15(\mathrm{~s}, 2 \mathrm{H}), 7.92(\mathrm{~d}$, $J=8.5 \mathrm{~Hz}, 2 \mathrm{H}), 8.07(\mathrm{~d}, J=8.5 \mathrm{~Hz}, 2 \mathrm{H}), 8.36$ (br s, 1H), $8.74(\mathrm{~s}, 1 \mathrm{H}) ;{ }^{13} \mathrm{C}-\mathrm{NMR}\left(\mathrm{DMSO}-d_{6}\right) \delta 13.6,19.5$, 31.0, 38.5, 51.9, 110.0, 118.8, 124.6, 125.6, 133.0, 135.2, 144.5, 164.9; LC-MS (ESI) $m / z 284\left([\mathrm{M}+1]^{+}\right)$.

N-Butyl-2-[4-(thiophen-3-yl)-1H-1,2,3-triazol-1-yl]acetamide (1ead). White solid; Yield: 80\%. ${ }^{1} \mathrm{H}-\mathrm{NMR}$ $\left(\mathrm{DMSO}_{6}\right) \delta 0.88(\mathrm{t}, J=7.3 \mathrm{~Hz}, 3 \mathrm{H}), 1.31(\mathrm{~m}, 2 \mathrm{H}), 1.43(\mathrm{~m}, 2 \mathrm{H}), 3.11(\mathrm{~m}, 2 \mathrm{H}), 5.10(\mathrm{~s}, 2 \mathrm{H}), 7.53(\mathrm{dd}$, $J=1.3,5.0 \mathrm{~Hz}, 1 \mathrm{H}), 7.64(\mathrm{dd}, J=2.9,4.9 \mathrm{~Hz}, 1 \mathrm{H}), 7.86(\mathrm{dd}, J=1.2,2.9 \mathrm{~Hz}, 1 \mathrm{H}), 8.32(\mathrm{br} \mathrm{s}, 1 \mathrm{H}), 8.38(\mathrm{~s}$, $1 \mathrm{H}) ;{ }^{13} \mathrm{C}-\mathrm{NMR}\left(\mathrm{DMSO}-d_{6}\right) \delta 13.6,13.7,19.5,21.6,24.6,31.0,31.1,38.4,51.5,123.2,146.5,165.3$; LC-MS (ESI) $m / z 265\left([\mathrm{M}+1]^{+}\right)$.

N-Butyl-2-[4-(pyridin-2-yl)-1H-1,2,3-triazol-1-yl]acetamide (1eae). Light yellow solid; Yield: $89 \%$. ${ }^{1} \mathrm{H}-\mathrm{NMR}\left(\mathrm{DMSO}-d_{6}\right) \delta 0.88(\mathrm{t}, J=7.3 \mathrm{~Hz}, 3 \mathrm{H}), 1.31(\mathrm{~m}, 2 \mathrm{H}), 1.43(\mathrm{~m}, 2 \mathrm{H}), 3.11(\mathrm{~m}, 2 \mathrm{H}), 5.15(\mathrm{~s}, 2 \mathrm{H})$, $7.34(\mathrm{dd}, J=5.1,6.6 \mathrm{~Hz}, 1 \mathrm{H}), 7.89(\mathrm{dt}, J=1.7,7.7 \mathrm{~Hz}, 1 \mathrm{H}), 8.03(\mathrm{~d}, J=7.8 \mathrm{~Hz}, 1 \mathrm{H}), 8.31(\mathrm{br} \mathrm{s}, 1 \mathrm{H}), 8.51$ $(\mathrm{s}, 1 \mathrm{H}), 8.60(\mathrm{~d}, J=4.4 \mathrm{~Hz}, 1 \mathrm{H}) ;{ }^{13} \mathrm{C}-\mathrm{NMR}\left(\mathrm{DMSO}_{6}\right) \delta 13.6,19.5,31.0,38.4,51.8,119.3,122.9,124.8$, 137.1, 146.9, 149.6, 150.0, 165.0; LC-MS (ESI) $m / z 260\left([\mathrm{M}+1]^{+}\right)$.

N-Butyl-2-(4-butyl-1H-1,2,3-triazol-1-yl)acetamide (1eaf). White solid; Yield: 89\%. ${ }^{1} \mathrm{H}-\mathrm{NMR}$ (DMSO- $\left.d_{6}\right)$ $\delta 0.87(\mathrm{t}, J=7.3 \mathrm{~Hz}, 3 \mathrm{H}), 0.89(\mathrm{t}, J=7.4 \mathrm{~Hz}, 3 \mathrm{H}), 1.31(\mathrm{~m}, 4 \mathrm{H}), 1.40(\mathrm{~m}, 2 \mathrm{H}), 1.57(\mathrm{~m}, 2 \mathrm{H}), 2.61(\mathrm{t}$, $J=7.6 \mathrm{~Hz}, 2 \mathrm{H}), 3.09(\mathrm{~m}, 2 \mathrm{H}), 4.99(\mathrm{~s}, 2 \mathrm{H}), 7.76(\mathrm{~s}, 1 \mathrm{H}), 8.23$ (br s, 1H); ${ }^{13} \mathrm{C}-\mathrm{NMR}\left(\mathrm{DMSO}-d_{6}\right) \delta 13.6$, 19.5, 31.0, 38.5, 51.7, 120.7, 122.7, 125.8, 127.1, 132.1, 142.7, 165.1; LC-MS (ESI) m/z $239\left([\mathrm{M}+1]^{+}\right)$.

N-Isopropyl-2-(4-phenyl-1H-1,2,3-triazol-1-yl)acetamide (1faa). White solid; Yield: 79\%. ${ }^{1} \mathrm{H}-\mathrm{NMR}$ $\left.(\text { DMSO-d })_{6}\right) \delta 1.10(\mathrm{~d}, J=6.6 \mathrm{~Hz}, 6 \mathrm{H}), 3.86(\mathrm{~m}, 1 \mathrm{H}), 5.08(\mathrm{~s}, 2 \mathrm{H}), 7.33(\mathrm{t}, J=7.4 \mathrm{~Hz}, 1 \mathrm{H}), 7.45(\mathrm{t}$, $J=7.7 \mathrm{~Hz}, 2 \mathrm{H}), 7.86(\mathrm{~d}, J=7.1 \mathrm{~Hz}, 1 \mathrm{H}), 8.29(\mathrm{~d}, J=7.4 \mathrm{~Hz}, 1 \mathrm{H}), 8.51(\mathrm{~s}, 1 \mathrm{H}) ;{ }^{13} \mathrm{C}-\mathrm{NMR}\left(\mathrm{DMSO}_{6}\right) \delta$ $22.3,40.9,51.8,122.9,125.1,127.8,128.9,130.8,146.1,164.2$; LC-MS (ESI) $m / z 245\left([\mathrm{M}+1]^{+}\right)$.

N-Isopropyl-2-[4-(4-methoxyphenyl)-1H-1,2,3-triazol-1-yl]acetamide (1fab). Light yellow solid; Yield: 87\%. ${ }^{1} \mathrm{H}-\mathrm{NMR}\left(\mathrm{DMSO}-\mathrm{d}_{6}\right) \delta 1.10(\mathrm{~d}, J=6.6 \mathrm{~Hz}, 6 \mathrm{H}), 3.79(\mathrm{~s}, 3 \mathrm{H}), 3.85(\mathrm{~m}, 1 \mathrm{H}), 5.05(\mathrm{~s}, 2 \mathrm{H}), 7.01$ $(\mathrm{d}, J=8.8 \mathrm{~Hz}, 2 \mathrm{H}), 7.78(\mathrm{~d}, J=8.8 \mathrm{~Hz}, 2 \mathrm{H}), 8.28(\mathrm{~d}, J=7.6 \mathrm{~Hz}, 1 \mathrm{H}), 8.39(\mathrm{~s}, 1 \mathrm{H}) ;{ }^{13} \mathrm{C}-\mathrm{NMR}\left(\mathrm{DMSO}-d_{6}\right)$ $\delta 22.3,40.9,51.8,55.1,114.3,122.0,123.4,126.5,146.0,159.0,164.3 ;$ LC-MS (ESI) $m / z 275\left([\mathrm{M}+1]^{+}\right)$.

2-[4-(4-Cyanophenyl)-1H-1,2,3-triazol-1-yl]-N-isopropyl-acetamide (1fac). Light brown solid; Yield: 75\%. ${ }^{1} \mathrm{H}-\mathrm{NMR}\left(\mathrm{DMSO}-\mathrm{d}_{6}\right) \delta 1.10(\mathrm{~d}, J=6.6 \mathrm{~Hz}, 6 \mathrm{H}), 3.85(\mathrm{~m}, 1 \mathrm{H}), 5.12(\mathrm{~s}, 2 \mathrm{H}), 7.92(\mathrm{~d}, J=8.6 \mathrm{~Hz}, 2 \mathrm{H}), 8.07$ $(\mathrm{d}, J=8.6 \mathrm{~Hz}, 2 \mathrm{H}), 8.32(\mathrm{~d}, \mathrm{~J}=7.4 \mathrm{~Hz}, 1 \mathrm{H}), 8.73(\mathrm{~s}, 1 \mathrm{H}) ;{ }^{13} \mathrm{C}-\mathrm{NMR}\left(\mathrm{DMSO}-d_{6}\right) \delta 22.2,41.0,51.9,110.1$, $118.8,124.7,125.7,133.0,135.2,144.5,164.0$; LC-MS (ESI) $m / z 270\left([\mathrm{M}+1]^{+}\right)$.

N-Isopropyl-2-[4-(thiophen-3-yl)-1H-1,2,3-triazol-1-yl]acetamide (1fad). White solid; Yield: 77\%. ${ }^{1} \mathrm{H}-\mathrm{NMR}\left(\mathrm{DMSO}-\mathrm{d}_{6}\right) \delta 1.10(\mathrm{~d}, J=6.6 \mathrm{~Hz}, 6 \mathrm{H}), 3.85(\mathrm{~m}, 1 \mathrm{H}), 5.07(\mathrm{~s}, 2 \mathrm{H}), 7.53(\mathrm{dd}, J=1.2,5.0 \mathrm{~Hz}$, $1 \mathrm{H}), 7.64(\mathrm{dd}, J=2.9,5.0 \mathrm{~Hz}, 1 \mathrm{H}), 7.86(\mathrm{dd}, J=1.2,2.9 \mathrm{~Hz}, 1 \mathrm{H}), 8.28(\mathrm{~d}, J=7.0 \mathrm{~Hz}, 1 \mathrm{H}), 8.37(\mathrm{~s}, 1 \mathrm{H})$; ${ }^{13}$ C-NMR (DMSO- $\left.d_{6}\right) \delta 22.3,40.9,51.7,120.7,122.7,125.8,127.1,132.1,142.7,164.2 ;$ LC-MS (ESI) $m / z$ $251\left([\mathrm{M}+1]^{+}\right)$.

N-Isopropyl-2-[4-(pyridin-2-yl)-1H-1,2,3-triazol-1-yl]acetamide (1fae). White solid; Yield: $84 \% .{ }^{1} \mathrm{H}-\mathrm{NMR}$ $\left(\mathrm{DMSO}_{-}\right) \delta 1.10(\mathrm{~d}, J=6.6 \mathrm{~Hz}, 6 \mathrm{H}), 3.85(\mathrm{~m}, 1 \mathrm{H}), 5.11(\mathrm{~s}, 2 \mathrm{H}), 7.34(\mathrm{ddd}, J=1.2,4.8,7.5 \mathrm{~Hz}, 1 \mathrm{H})$, $7.89(\mathrm{dt}, J=1.8,7.7 \mathrm{~Hz}, 1 \mathrm{H}), 8.03(\mathrm{td}, J=1.0,7.9 \mathrm{~Hz}, 1 \mathrm{H}), 8.28(\mathrm{~d}, J=7.5 \mathrm{~Hz}, 1 \mathrm{H}), 8.51(\mathrm{~s}, 1 \mathrm{H}), 8.60(\mathrm{~m}$, $1 \mathrm{H}) ;{ }^{13} \mathrm{C}-\mathrm{NMR}\left(\mathrm{DMSO}-d_{6}\right) \delta 22.3,41.0,51.8,119.4,122.9,124.8,137.2,146.9,149.6,150.0,164.2$; LC-MS (ESI) $m / z 246\left([\mathrm{M}+1]^{+}\right)$. 
2-(4-Butyl-1H-1,2,3-triazol-1-yl)-N-isopropylacetamide (1faf). White solid; Yield: $83 \% .{ }^{1} \mathrm{H}-\mathrm{NMR}$ $\left(\right.$ DMSO- $\left.d_{6}\right) \delta 0.89(\mathrm{t}, J=7.3 \mathrm{~Hz}, 3 \mathrm{H}), 1.08(\mathrm{~d}, J=6.6 \mathrm{~Hz}, 3 \mathrm{H}), 1.32(\mathrm{~m}, 2 \mathrm{H}), 1.57(\mathrm{~m}, 2 \mathrm{H}), 2.61(\mathrm{~d}$, $J=7.6 \mathrm{~Hz}, 2 \mathrm{H}), 3.82(\mathrm{~m}, 1 \mathrm{H}), 4.96(\mathrm{~s}, 2 \mathrm{H}), 7.76(\mathrm{~s}, 1 \mathrm{H}), 8.21(\mathrm{~d}, J=7.4 \mathrm{~Hz}, 1 \mathrm{H}) ;{ }^{13} \mathrm{C}-\mathrm{NMR}\left(\mathrm{DMSO}-d_{6}\right) \delta$ $13.7,21.7,22.2,24.6,31.1,40.8,51.5,123.2,146.5,164.4$; LC-MS (ESI) $m / z 251\left([\mathrm{M}+1]^{+}\right)$.

N-(2-Methoxyphenyl)-2-(4-phenyl-1H-1,2,3-triazol-1-yl)propanamide (1dba). Yellow solid; Yield: 88\%. ${ }^{1} \mathrm{H}-\mathrm{NMR}\left(\mathrm{CDCl}_{3}\right) \delta 1.98(\mathrm{~d}, J=7.3 \mathrm{~Hz}, 3 \mathrm{H}), 3.81(\mathrm{~s}, 3 \mathrm{H}), 5.50(\mathrm{q}, J=7.2 \mathrm{~Hz}, 1 \mathrm{H}), 6.84(\mathrm{dd}, J=1.2$, $8.2 \mathrm{~Hz}, 1 \mathrm{H}), 6.95(\mathrm{dt}, J=1.1,7.8 \mathrm{~Hz}, 1 \mathrm{H}), 7.06(\mathrm{dt}, J=1.5,7.9 \mathrm{~Hz}, 1 \mathrm{H}), 7.36(\mathrm{~m}, 1 \mathrm{H}), 7.43-7.46(\mathrm{~m}$, $2 \mathrm{H}), 7.85-7.87(\mathrm{~m}, 2 \mathrm{H}), 8.01(\mathrm{~s}, 1 \mathrm{H}), 8.28(\mathrm{dd}, J=1.5,8.1 \mathrm{~Hz}, 1 \mathrm{H}), 8.32(\mathrm{~s}, 1 \mathrm{H}) ;{ }^{13} \mathrm{C}-\mathrm{NMR}\left(\mathrm{DMSO}-d_{6}\right)$ $\delta 18.0,55.8,58.5,111.3,120.2,120.8,122.1,125.1,127.8,128.9,146.1,149.9,167.6$; LC-MS (ESI) $\mathrm{m} / z 323$ $\left([\mathrm{M}+1]^{+}\right)$.

$\mathrm{N}$-(2-Methoxyphenyl)-2-[4-(4-methoxyphenyl)-1H-1,2,3-triazol-1-yl]propanamide (1dbb). White solid; Yield: 91\%. ${ }^{1} \mathrm{H}-\mathrm{NMR}\left(\mathrm{CDCl}_{3}\right) \delta 3.79(\mathrm{~s}, 3 \mathrm{H}), 3.85(\mathrm{~s}, 3 \mathrm{H}), 5.25(\mathrm{~s}, 2 \mathrm{H}), 6.84(\mathrm{dd}, J=1.0,8.2 \mathrm{~Hz}, 1 \mathrm{H}), 6.95$ $(\mathrm{dt}, J=1.1,7.7 \mathrm{~Hz}, 1 \mathrm{H}), 6.98(\mathrm{~d}, J=8.9 \mathrm{~Hz}, 2 \mathrm{H}), 7.07(\mathrm{dt}, J=1.5,7.9 \mathrm{~Hz}, 1 \mathrm{H}), 7.79(\mathrm{~d}, J=8.9 \mathrm{~Hz}, 2 \mathrm{H})$, $7.90(\mathrm{~s}, 1 \mathrm{H}), 8.24(\mathrm{~s}, 1 \mathrm{H}), 8.27(\mathrm{dd}, J=1.5,8.1 \mathrm{~Hz}, 1 \mathrm{H}) ;{ }^{13} \mathrm{C}-\mathrm{NMR}\left(\mathrm{DMSO}-d_{6}\right) \delta 18.0,55.1,55.8,58.5$, $111.3,114.3,119.9,120.2,122.1,123.4,125.2,126.38,126.44,146.0,149.9,158.9,167.6$; LC-MS (ESI) $\mathrm{m} / \mathrm{z}$ $353\left([\mathrm{M}+1]^{+}\right)$.

2-[4-(4-Cyanophenyl)-1H-1,2,3-triazol-1-yl]-N-(2-methoxyphenyl)propanamide (1dbc). Light yellow solid; Yield: $87 \% .{ }^{1} \mathrm{H}-\mathrm{NMR}\left(\mathrm{CDCl}_{3}\right) \delta 1.98(\mathrm{~d}, J=7.2 \mathrm{~Hz}, 3 \mathrm{H}), 5.53(\mathrm{q}, J=7.2 \mathrm{~Hz}, 1 \mathrm{H}), 6.87(\mathrm{~d}, J=8.1 \mathrm{~Hz}, 1 \mathrm{H})$, $6.96(\mathrm{t}, J=7.8 \mathrm{~Hz}, 1 \mathrm{H}), 7.08(\mathrm{dt}, J=1.4,7.9 \mathrm{~Hz}, 1 \mathrm{H}), 7.73(\mathrm{~d}, J=8.5 \mathrm{~Hz}, 2 \mathrm{H}), 7.98(\mathrm{~d}, J=8.5 \mathrm{~Hz}, 2 \mathrm{H})$, $8.15(\mathrm{~s}, 1 \mathrm{H}), 8.27(\mathrm{dd}, J=1.4,8.1 \mathrm{~Hz}, 1 \mathrm{H}), 8.30(\mathrm{~s}, 1 \mathrm{H}) ;{ }^{13} \mathrm{C}-\mathrm{NMR}\left(\mathrm{DMSO}-d_{6}\right) \delta 18.0,55.8,58.7,110.1$, $111.4,118.8,120.2,122.2,122.7,125.3,125.6,126.3,133.0,135.2,144.6,150.0,167.5$; LC-MS (ESI) $\mathrm{m} / z 348$ $\left([\mathrm{M}+1]^{+}\right)$.

N-(2-Methoxyphenyl)-2-[4-(thiophen-3-yl)-1H-1,2,3-triazol-1-yl]propanamide (1dbd). Light brown oil; Yield: $80 \% .{ }^{1} \mathrm{H}-\mathrm{NMR}\left(\mathrm{CDCl}_{3}\right) \delta 1.94(\mathrm{~d}, J=7.3 \mathrm{~Hz}, 3 \mathrm{H}), 3.78(\mathrm{~s}, 3 \mathrm{H}), 5.52(\mathrm{q}, J=7.2 \mathrm{~Hz}, 2 \mathrm{H}), 6.83$ $(\mathrm{dd}, J=1.1,8.2 \mathrm{~Hz}, 1 \mathrm{H}), 6.90(\mathrm{dt}, J=1.2,8.4 \mathrm{~Hz}, 1 \mathrm{H}), 7.05(\mathrm{~m}, 1 \mathrm{H}), 7.38(\mathrm{dd}, J=3.0,5.0 \mathrm{~Hz}, 1 \mathrm{H}), 7.47$ $(\mathrm{dd}, J=1.2,5.0 \mathrm{~Hz}, 1 \mathrm{H}), 7.71(\mathrm{dd}, J=1.2,3.0 \mathrm{~Hz}, 1 \mathrm{H}), 7.95(\mathrm{~s}, 1 \mathrm{H}), 8.26(\mathrm{dd}, J=1.5,8.1 \mathrm{~Hz}, 1 \mathrm{H}), 8.38$ (br s, $1 \mathrm{H}$ ); ${ }^{13} \mathrm{C}-\mathrm{NMR}$ (DMSO-d $\left.d_{6}\right) \delta 18.1,55.8,58.4,111.4,120.3,120.6,120.7,122.1,125.2,125.8,127.1$, 132.1, 142.7, 149.9, 167.6; LC-MS (ESI) $m / z 329\left([\mathrm{M}+1]^{+}\right)$.

N-(2-Methoxyphenyl)-2-[4-(pyridin-2-yl)-1H-1,2,3-triazol-1-yl]propanamide (1dbe). Colorless oil; Yield: 83\%. ${ }^{1} \mathrm{H}-\mathrm{NMR}\left(\mathrm{CDCl}_{3}\right) \delta 1.97(\mathrm{~d}, J=7.2 \mathrm{~Hz}, 3 \mathrm{H}), 3.78(\mathrm{~s}, 3 \mathrm{H}), 5.53(\mathrm{q}, J=7.2 \mathrm{~Hz}, 1 \mathrm{H}), 6.82(\mathrm{dd}, J=1.1$, $8.2 \mathrm{~Hz}, 1 \mathrm{H}), 6.92(\mathrm{dt}, J=1.1,7.8 \mathrm{~Hz}, 1 \mathrm{H}), 7.04(\mathrm{dt}, J=1.5,7.9 \mathrm{~Hz}, 1 \mathrm{H}), 7.24(\mathrm{ddd}, J=1.2,4.9,7.6 \mathrm{~Hz}$, $1 \mathrm{H}), 7.78(\mathrm{dt}, J=1.8,7.8 \mathrm{~Hz}, 1 \mathrm{H}), 8.18(\mathrm{dd}, J=0.9,8.8 \mathrm{~Hz}, 1 \mathrm{H}), 8.26(\mathrm{dd}, J=1.6,8.1 \mathrm{~Hz}, 1 \mathrm{H}), 8.36(\mathrm{br}$ $\mathrm{s}, 1 \mathrm{H}), 8.39(\mathrm{~s}, 1 \mathrm{H}), 8.59(\mathrm{ddd}, J=0.9,1.7,4.8 \mathrm{~Hz}, 1 \mathrm{H}) ;{ }^{13} \mathrm{C}-\mathrm{NMR}\left(\mathrm{DMSO}-d_{6}\right) \delta 18.0,55.8,58.7,111.4$, 119.4, 120.3, 122.2, 122.7, 123.0, 125.2, 126.4, 137.2, 147.0, 149.6, 149.9, 150.0, 167.5; LC-MS (ESI) $\mathrm{m} / \mathrm{z} 324$ $\left([\mathrm{M}+1]^{+}\right)$.

N-(2-Methoxyphenyl)-2-[4-(3-methoxyphenyl)-1H-1,2,3-triazol-1-yl]propanamide (1dbg). Light yellow oil; Yield: 94\%. ${ }^{1} \mathrm{H}-\mathrm{NMR}\left(\mathrm{CDCl}_{3}\right) \delta 1.95(\mathrm{~d}, J=7.2 \mathrm{~Hz}, 3 \mathrm{H}), 3.77(\mathrm{~s}, 3 \mathrm{H}), 3.85(\mathrm{~s}, 3 \mathrm{H}), 5.53(\mathrm{q}, J=7.2 \mathrm{~Hz}$, $1 \mathrm{H}), 6.82(\mathrm{dd}, \mathrm{J}=1.2,8.2 \mathrm{~Hz}, 1 \mathrm{H}), 6.89(\mathrm{ddd}, \mathrm{J}=1.0,2.6,8.2 \mathrm{~Hz}, 1 \mathrm{H}), 6.92(\mathrm{dt}, J=1.2, .8 \mathrm{~Hz}, 1 \mathrm{H}), 7.04$ $(\mathrm{dt}, J=1.6,7.9 \mathrm{~Hz}, 1 \mathrm{H}), 7.32(\mathrm{t}, J=7.9 \mathrm{~Hz}, 1 \mathrm{H}), 7.39(\mathrm{td}, J=1.3,7.7 \mathrm{~Hz}, 1 \mathrm{H}), 7.46(\mathrm{dd}, J=1.5,2.5 \mathrm{~Hz}$, $1 \mathrm{H}), 8.04(\mathrm{~s}, 1 \mathrm{H}), 8.26(\mathrm{dd}, J=1.6,8.1 \mathrm{~Hz}, 1 \mathrm{H}), 8.39(\mathrm{br} \mathrm{s}, 1 \mathrm{H}) ;{ }^{13} \mathrm{C}-\mathrm{NMR}\left(\mathrm{DMSO}-d_{6}\right) \delta 18.0,55.1,55.8$, 58.5, 110.3, 111.4, 113.7, 117.4, 120.3, 121.2, 122.1, 125.2, 126.4, 130.0, 132.1, 146.0, 149.9, 159.7, 167.6; LC-MS (ESI) $m / z 353\left([\mathrm{M}+1]^{+}\right)$.

N-(2-Methoxyphenyl)-2-[4-(2-methoxyphenyl)-1H-1,2,3-triazol-1-yl]propanamide (1dbh). Light yellow oil; Yield: 89\%. ${ }^{1} \mathrm{H}-\mathrm{NMR}\left(\mathrm{CDCl}_{3}\right) \delta 1.98(\mathrm{~d}, J=7.3 \mathrm{~Hz}, 3 \mathrm{H}), 3.75(\mathrm{~s}, 3 \mathrm{H}), 3.92(\mathrm{~s}, 3 \mathrm{H}), 5.51(\mathrm{q}, J=7.3 \mathrm{~Hz}$, $1 \mathrm{H}), 6.80(\mathrm{dd}, J=1.2,8.2 \mathrm{~Hz}, 1 \mathrm{H}), 6.92(\mathrm{dt}, J=1.2,7.8 \mathrm{~Hz}, 1 \mathrm{H}), 6.97(\mathrm{dd}, J=0.6,8.3 \mathrm{~Hz}, 1 \mathrm{H}), 7.03(\mathrm{dt}$, $J=1.5,7.9 \mathrm{~Hz}, 1 \mathrm{H}), 7.08(\mathrm{dt}, J=1.0,7.5 \mathrm{~Hz}, 1 \mathrm{H}), 7.32(\mathrm{ddd}, J=1.7,7.4,8.3 \mathrm{~Hz}, 1 \mathrm{H}), 8.25(\mathrm{~s}, 1 \mathrm{H}), 8.27$ $(\mathrm{dd}, J=1.6,8.1 \mathrm{~Hz}, 1 \mathrm{H}), 8.37(\mathrm{dd}, J=1.7,7.7 \mathrm{~Hz}, 1 \mathrm{H}), 8.41(\mathrm{br} \mathrm{s}, 1 \mathrm{H}) ;{ }^{13} \mathrm{C}-\mathrm{NMR}\left(\mathrm{DMSO}-d_{6}\right) \delta$ 18.1. 55.5. 
55.8. 58.5. 111.4. 111.5. 119.0. 120.3. 120.6. 122.1. 123.0. 125.2. 126.4. 126.6. 128.9. 141.7. 149.9. 155.4. 167.6; LC-MS (ESI) $m / z 353\left([\mathrm{M}+1]^{+}\right)$.

N-(2-Methoxyphenyl)-2-(4-p-tolyl-1H-1,2,3-triazol-1-yl)propanamide (1dbi). Light yellow solid; Yield: 94\%. ${ }^{1} \mathrm{H}-\mathrm{NMR}\left(\mathrm{CDCl}_{3}\right) \delta 1.97(\mathrm{~d}, J=7.3 \mathrm{~Hz}, 3 \mathrm{H}), 2.38(\mathrm{~s}, 3 \mathrm{H}), 3.80(\mathrm{~s}, 3 \mathrm{H}), 5.49(\mathrm{q}, J=7.2 \mathrm{~Hz}, 1 \mathrm{H})$, $6.84(\mathrm{dd}, J=1.0,8.2 \mathrm{~Hz}, 1 \mathrm{H}), 6.94(\mathrm{dt}, J=1.1,7.8 \mathrm{~Hz}, 1 \mathrm{H}), 7.06(\mathrm{dt}, J=1.5,7.9 \mathrm{~Hz}, 1 \mathrm{H}), 7.25(\mathrm{~d}$, $J=7.9 \mathrm{~Hz}, 2 \mathrm{H}), 7.74(\mathrm{~d}, J=8.2 \mathrm{~Hz}, 2 \mathrm{H}), 7.97(\mathrm{~s}, 1 \mathrm{H}), 8.28(\mathrm{dd}, J=1.5,8.1 \mathrm{~Hz}, 1 \mathrm{H}), 8.33(\mathrm{br} \mathrm{s}, 1 \mathrm{H})$; ${ }^{13}$ C-NMR (DMSO- $d_{6}$ ) $\delta 18.0,20.8,55.8,58.5,111.3,120.2,120.4,122.1,125.0,125.2,126.4,128.0,129.4$, 137.1, 146.2, 149.9, 167.6; LC-MS (ESI) $m / z 337\left([\mathrm{M}+1]^{+}\right)$.

N-(2-Methoxyphenyl)-2-(4-m-tolyl-1H-1,2,3-triazol-1-yl)propanamide (1dbj). Light yellow solid; Yield: 83\%. ${ }^{1} \mathrm{H}-\mathrm{NMR}\left(\mathrm{CDCl}_{3}\right) \delta 1.97(\mathrm{~d}, J=7.3 \mathrm{~Hz}, 3 \mathrm{H}), 2.41(\mathrm{~s}, 3 \mathrm{H}), 3.80(\mathrm{~s}, 3 \mathrm{H}), 5.51(\mathrm{q}, J=7.2 \mathrm{~Hz}, 1 \mathrm{H}), 6.84$ $(\mathrm{dd}, J=1.1,8.2 \mathrm{~Hz}, 1 \mathrm{H}), 6.94(\mathrm{dt}, J=1.2,7.8 \mathrm{~Hz}, 1 \mathrm{H}), 7.06(\mathrm{dt}, J=1.5,7.9 \mathrm{~Hz}, 1 \mathrm{H}), 7.17(\mathrm{~d}, J=7.6 \mathrm{~Hz}$, $1 \mathrm{H}), 7.32(\mathrm{t}, J=7.7 \mathrm{~Hz}, 1 \mathrm{H}), 7.63(\mathrm{~d}, J=7.7 \mathrm{~Hz}, 1 \mathrm{H}), 7.71(\mathrm{~s}, 1 \mathrm{H}), 8.00(\mathrm{~s}, 1 \mathrm{H}), 8.28(\mathrm{dd}, J=1.5,8.1 \mathrm{~Hz}$, 1H), 8.34 (br s, $1 \mathrm{H}$ ); ${ }^{13} \mathrm{C}-\mathrm{NMR}$ (DMSO- $d_{6}$ ) $\delta 18.0,20.8,55.8,58.5,111.3,120.2,120.4,122.1,125.0,125.1$, 126.4, 128.0, 129.4, 137.1, 146.2, 149.9, 167.6; LC-MS (ESI) $m / z 337\left([\mathrm{M}+1]^{+}\right)$.

N-(2-Methoxyphenyl)-2-(4-o-tolyl-1H-1,2,3-triazol-1-yl)propanamide (1dbk). Light yellow solid; Yield: 74\%. ${ }^{1} \mathrm{H}-\mathrm{NMR}$ (acetone- $\left.d_{6}\right) \delta 1.97(\mathrm{~d}, J=7.2 \mathrm{~Hz}, 3 \mathrm{H}), 2.52(\mathrm{~s}, 3 \mathrm{H}), 3.84(\mathrm{~s}, 3 \mathrm{H}), 5.85(\mathrm{q}, J=7.3 \mathrm{~Hz}$, $1 \mathrm{H}), 6.93(\mathrm{~m}, 1 \mathrm{H}), 7.02(\mathrm{~d}, J=7.1 \mathrm{~Hz}, 1 \mathrm{H}), 7.25-7.32(\mathrm{~m}, 3 \mathrm{H}), 7.82(\mathrm{~m}, 1 \mathrm{H}), 8.26(\mathrm{~m}, 1 \mathrm{H}), 8.41(\mathrm{~s}, 1 \mathrm{H})$; ${ }^{13}$ C-NMR (DMSO- $d_{6}$ ) $\delta 18.5,21.5,56.3,59.1,111.8,120.8,122.6,123.2,125.7,126.5,127.0,128.3,128.8$, 130.5, 131.3, 135.5, 146.0, 150.4, 166.3; LC-MS (ESI) $\mathrm{m} / \mathrm{z} 337\left([\mathrm{M}+1]^{+}\right)$.

2-\{4-[4-(Dimethylamino)phenyl]-1H-1,2,3-triazol-1-yl\}-N-(2-methoxyphenyl)propanamide (1dbl). Brown solid; Yield: 82\%. ${ }^{1} \mathrm{H}-\mathrm{NMR}\left(\mathrm{CDCl}_{3}\right) \delta 1.96(\mathrm{~d}, J=7.3 \mathrm{~Hz}, 3 \mathrm{H}), 3.00(\mathrm{~s}, 6 \mathrm{H}), 3.79(\mathrm{~s}, 3 \mathrm{H}), 5.47(\mathrm{q}$, $J=7.2 \mathrm{~Hz}, 2 \mathrm{H}), 6.77(\mathrm{~d}, J=9.0 \mathrm{~Hz}, 2 \mathrm{H}), 6.83(\mathrm{dd}, J=1.1,8.2 \mathrm{~Hz}, 1 \mathrm{H}), 6.94(\mathrm{dt}, J=1.2,7.8 \mathrm{~Hz}, 1 \mathrm{H}), 7.05$ $(\mathrm{dt}, J=1.5,7.9 \mathrm{~Hz}, 1 \mathrm{H}), 7.72(\mathrm{~d}, J=8.9 \mathrm{~Hz}, 2 \mathrm{H}), 7.85(\mathrm{~s}, 1 \mathrm{H}), 8.28(\mathrm{dd}, J=1.6,8.1 \mathrm{~Hz}, 1 \mathrm{H}), 8.32(\mathrm{br} \mathrm{s}$, 1H); ${ }^{13}$ C-NMR (DMSO- $d_{6}$ ) $\delta 18.0,40.0,55.8,58.4,111.3,112.3,118.7,118.9,120.3,122.0,125.1,126.0$, 126.4, 146.7, 149.9, 150.0, 167.7; LC-MS (ESI) $m / z 366\left([\mathrm{M}+1]^{+}\right)$.

N-(2-Methoxyphenyl)-2-[4-(4-phenoxyphenyl)-1H-1,2,3-triazol-1-yl]propanamide (1dbm). Light yellow oil; Yield: 87\%. ${ }^{1} \mathrm{H}-\mathrm{NMR}\left(\mathrm{CDCl}_{3}\right) \delta 1.97(\mathrm{~d}, J=7.3 \mathrm{~Hz}, 3 \mathrm{H}), 3.80(\mathrm{~s}, 3 \mathrm{H}), 5.52(\mathrm{q}, J=7.2 \mathrm{~Hz}, 1 \mathrm{H}), 6.84$ $(\mathrm{dd}, J=1.2,8.2 \mathrm{~Hz}, 1 \mathrm{H}), 6.94(\mathrm{~m}, 1 \mathrm{H}), 7.02-7.08(\mathrm{~m}, 5 \mathrm{H}), 7.15(\mathrm{~m}, 1 \mathrm{H}), 7.34-7.37(\mathrm{~m}, 2 \mathrm{H}), 7.82(\mathrm{~d}$, $J=8.8 \mathrm{~Hz}, 2 \mathrm{H}), 7.99(\mathrm{~s}, 1 \mathrm{H}), 8.28(\mathrm{dd}, J=1.5,8.1 \mathrm{~Hz}, 1 \mathrm{H}), 8.37(\mathrm{br} \mathrm{s}, 1 \mathrm{H}) ;{ }^{13} \mathrm{C}-\mathrm{NMR}$ (DMSO- $\left.d_{6}\right) \delta 18.0$, 55.8, 80.0, 111.3, 118.2, 118.81, 118.86, 119.4, 120.3, 120.5, 123.6, 126.9, 130.1, 130.2, 133.6, 145.7, 149.9, 156.39, 156.42, 167.6; LC-MS (ESI) $m / z 415\left([\mathrm{M}+1]^{+}\right)$.

2-[4-(2,4-Difluorophenyl)-1H-1,2,3-triazol-1-yl]-N-(2-methoxyphenyl)propanamide (1dbn). Light yellow solid; Yield: 92\%. ${ }^{1} \mathrm{H}-\mathrm{NMR}\left(\mathrm{CDCl}_{3}\right) \delta 1.99(\mathrm{~d}, J=7.3 \mathrm{~Hz}, 3 \mathrm{H}), 3.82(\mathrm{~s}, 3 \mathrm{H}), 5.51(\mathrm{q}, J=7.2 \mathrm{~Hz}, 2 \mathrm{H})$, $6.85(\mathrm{dd}, J=1.2,8.2 \mathrm{~Hz}, 1 \mathrm{H}), 6.92(\mathrm{ddd}, J=2.4,8.7,11.0 \mathrm{~Hz}, 1 \mathrm{H}), 6.95(\mathrm{dt}, J=1.1,7.8 \mathrm{~Hz}, 1 \mathrm{H}), 7.01$ $(\mathrm{m}, 1 \mathrm{H}), 7.07(\mathrm{dt}, J=1.5,7.9 \mathrm{~Hz}, 1 \mathrm{H}), 8.12(\mathrm{~d}, J=3.5 \mathrm{~Hz}, 1 \mathrm{H}), 8.28(\mathrm{dd}, J=1.6,8.1 \mathrm{~Hz}, 1 \mathrm{H}), 8.29(\mathrm{dt}$, $J=6.5,8.6 \mathrm{~Hz}, 1 \mathrm{H}), 8.31(\mathrm{br} \mathrm{s}, 1 \mathrm{H}) ;{ }^{13} \mathrm{C}-\mathrm{NMR}\left(\mathrm{DMSO}_{6}\right) \delta$ 18.0, 55.8, 58.7, $104.6\left(\mathrm{t}, J_{\mathrm{CF}}=25.9 \mathrm{~Hz}\right), 111.4$, $112.30\left(\mathrm{dd}, J_{\mathrm{CF}}=21.6,3.6 \mathrm{~Hz}\right), 115.20\left(\mathrm{dd}, J_{\mathrm{CF}}=13.4,3.8 \mathrm{~Hz}\right), 120.3,122.2,122.8\left(\mathrm{~d}, J_{\mathrm{CF}}=10.3 \mathrm{~Hz}\right)$, $125.3,126.3,128.7\left(\mathrm{dd}, J_{\mathrm{CF}}=9.8,5.2 \mathrm{~Hz}\right), 139.1\left(\mathrm{~d}, J_{\mathrm{CF}}=2.6 \mathrm{~Hz}\right), 150.0,158.5\left(\mathrm{dd}, J_{\mathrm{CF}}=250.5,12.8 \mathrm{~Hz}\right)$, $161.86\left(\mathrm{dd}, J_{\mathrm{CF}}=247.8,12.6 \mathrm{~Hz}\right), 167.5$; LC-MS (ESI) $\mathrm{m} / \mathrm{z} 359\left([\mathrm{M}+1]^{+}\right)$.

2-(4-Benzyl-1H-1,2,3-triazol-1-yl)-N-(2-methoxyphenyl)propanamide (1dbo). Light yellow oil; Yield: 86\%. ${ }^{1} \mathrm{H}-\mathrm{NMR}\left(\mathrm{CDCl}_{3}\right) \delta 1.88(\mathrm{~d}, J=7.3 \mathrm{~Hz}, 3 \mathrm{H}), 3.77(\mathrm{~s}, 3 \mathrm{H}), 4.12(\mathrm{~s}, 2 \mathrm{H}), 5.39(\mathrm{q}, J=7.3 \mathrm{~Hz}, 1 \mathrm{H}), 6.83(\mathrm{dd}$, $J=1.1,8.2 \mathrm{~Hz}, 1 \mathrm{H}), 6.93(\mathrm{dt}, J=1.2,7.8 \mathrm{~Hz}, 1 \mathrm{H}), 7.05(\mathrm{dt}, J=1.5,7.9 \mathrm{~Hz}, 1 \mathrm{H}), 7.23(\mathrm{~m}, 1 \mathrm{H}), 7.26-7.31$ $(\mathrm{m}, 4 \mathrm{H}), 7.42(\mathrm{~s}, 1 \mathrm{H}), 8.25(\mathrm{dd}, J=1.5,8.1 \mathrm{~Hz}, 1 \mathrm{H}), 8.33(\mathrm{br} \mathrm{s}, 1 \mathrm{H}) ;{ }^{13} \mathrm{C}-\mathrm{NMR}$ (DMSO-d $\left.d_{6}\right) \delta 18.0,31.2$, $55.7,58.3,111.3,120.2,121.8,122.0,125.1,126.1,126.4,128.4,128.5,139.6,145.8,149.9,167.6$; LC-MS (ESI) $m / z 337\left([\mathrm{M}+1]^{+}\right)$.

N-Butyl-2-(4-phenyl-1H-1,2,3-triazol-1-yl)propanamide (1eba). White solid; Yield: 83\%. ${ }^{1} \mathrm{H}-\mathrm{NMR}$ $\left(\right.$ DMSO- $\left._{6}\right) \delta 0.86(\mathrm{t}, J=7.3 \mathrm{~Hz}, 3 \mathrm{H}), 1.27(\mathrm{~m}, 2 \mathrm{H}), 1.41(\mathrm{~m}, 2 \mathrm{H}), 1.71(\mathrm{~d}, J=7.2 \mathrm{~Hz}, 3 \mathrm{H}), 3.10(\mathrm{~m}$, 
$2 \mathrm{H}), 5.38(\mathrm{q}, J=7.2 \mathrm{~Hz}, 1 \mathrm{H}), 7.33(\mathrm{t}, J=7.4 \mathrm{~Hz}, 1 \mathrm{H}), 7.31(\mathrm{t}, J=7.7 \mathrm{~Hz}, 2 \mathrm{H}), 7.88(\mathrm{~d}, J=7.1 \mathrm{~Hz}, 2 \mathrm{H})$, $8.38(\mathrm{br} \mathrm{s}, 1 \mathrm{H}), 8.68(\mathrm{~s}, 1 \mathrm{H}) ;{ }^{13} \mathrm{C}-\mathrm{NMR}\left(\mathrm{DMSO}-d_{6}\right) \delta 13.6,18.0,19.4,30.9,38.4,58.4,120.6,125.1,127.8$, $128.9,130.9,146.0,168.3$; LC-MS (ESI) $m / z 273\left([\mathrm{M}+1]^{+}\right)$.

N-Butyl-2-[4-(4-methoxyphenyl)-1H-1,2,3-triazol-1-yl]propanamide (1ebb). White solid; Yield: 81\%. ${ }^{1} \mathrm{H}-\mathrm{NMR}\left(\mathrm{DMSO}-d_{6}\right) \delta 0.86(\mathrm{t}, J=7.3 \mathrm{~Hz}, 3 \mathrm{H}), 1.27(\mathrm{~m}, 2 \mathrm{H}), 1.40(\mathrm{~m}, 2 \mathrm{H}), 1.70(\mathrm{~d}, J=7.2 \mathrm{~Hz}, 3 \mathrm{H})$, $3.79(\mathrm{~s}, 3 \mathrm{H}), 5.36(\mathrm{q}, J=7.2 \mathrm{~Hz}, 1 \mathrm{H}), 7.01(\mathrm{~d}, J=8.8 \mathrm{~Hz}, 2 \mathrm{H}), 7.80(\mathrm{~d}, J=8.8 \mathrm{~Hz}, 2 \mathrm{H}), 8.36(\mathrm{br} \mathrm{s}, 1 \mathrm{H}), 8.56$ $(\mathrm{s}, 1 \mathrm{H}) ;{ }^{13} \mathrm{C}-\mathrm{NMR}\left(\mathrm{DMSO}-d_{6}\right.$ ) $\delta$ 13.6, 18.0, 19.4, 30.9, 38.4, 55.1, 58.3, 114.3, 119.6, 123.5, 126.4, 146.0, 158.9, 168.4; LC-MS (ESI) $m / z 303\left([\mathrm{M}+1]^{+}\right)$.

N-Butyl-2-[4-(4-cyanophenyl)-1H-1,2,3-triazol-1-yl]propanamide (1ebc). White solid; Yield: 79\%. ${ }^{1} \mathrm{H}-\mathrm{NMR}$ (DMSO- $\left.d_{6}\right) \delta 0.86(\mathrm{t}, J=7.3 \mathrm{~Hz}, 3 \mathrm{H}), 1.27(\mathrm{~m}, 2 \mathrm{H}), 1.40(\mathrm{~m}, 2 \mathrm{H}), 1.72(\mathrm{~d}, J=7.2 \mathrm{~Hz}, 3 \mathrm{H})$, $3.09(\mathrm{~m}, 2 \mathrm{H}), 5.42(\mathrm{q}, J=7.2 \mathrm{~Hz}, 1 \mathrm{H}), 7.92(\mathrm{~d}, J=8.6 \mathrm{~Hz}, 2 \mathrm{H}), 8.09(\mathrm{~d}, J=8.6 \mathrm{~Hz}, 2 \mathrm{H}), 8.40(\mathrm{br} \mathrm{s}, 1 \mathrm{H})$, $8.92(\mathrm{~s}, 1 \mathrm{H}) ;{ }^{13} \mathrm{C}-\mathrm{NMR}\left(\mathrm{DMSO}-d_{6}\right) \delta$ 13.6, 18.0, 19.4, 30.9, 38.5, 58.5, 110.0, 118.8, 122.5, 125.6, 133.0, 135.3, 144.5, 168.2; LC-MS (ESI) $m / z 298\left([\mathrm{M}+1]^{+}\right)$.

N-Butyl-2-[4-(thiophen-3-yl)-1H-1,2,3-triazol-1-yl]propanamide (1ebd). Light yellow solid; Yield: 78\%. ${ }^{1} \mathrm{H}-\mathrm{NMR}\left(\mathrm{DMSO}-d_{6}\right) \delta 0.86(\mathrm{t}, J=7.3 \mathrm{~Hz}, 3 \mathrm{H}), 1.27(\mathrm{~m}, 2 \mathrm{H}), 1.40(\mathrm{~m}, 2 \mathrm{H}), 1.69(\mathrm{~d}, J=7.2 \mathrm{~Hz}, 3 \mathrm{H}), 3.08$ $(\mathrm{m}, 2 \mathrm{H}), 5.37(\mathrm{q}, J=7.1 \mathrm{~Hz}, 1 \mathrm{H}), 7.55(\mathrm{dd}, J=1.2,5.0 \mathrm{~Hz}, 1 \mathrm{H}), 7.64(\mathrm{dd}, J=2.9,5.0 \mathrm{~Hz}, 1 \mathrm{H}), 7.86(\mathrm{dd}$, $J=1.2,2.9 \mathrm{~Hz}, 1 \mathrm{H}), 8.38(\mathrm{br} \mathrm{s}, 1 \mathrm{H}), 8.55(\mathrm{~s}, 1 \mathrm{H}) ;{ }^{13} \mathrm{C}-\mathrm{NMR}\left(\mathrm{DMSO}-d_{6}\right) \delta 13.6,18.0,19.4,30.9,38.4,58.3$, $120.3,120.6,125.8,127.0,132.2,142.7,168.3$; LC-MS (ESI) $m / z 279\left([\mathrm{M}+1]^{+}\right)$.

N-Butyl-2-[4-(pyridin-2-yl)-1H-1,2,3-triazol-1-yl]propanamide (1ebe). White solid; Yield: $84 \% .{ }^{1} \mathrm{H}-\mathrm{NMR}$ $\left(\right.$ DMSO- $\left._{6}\right) \delta 0.86(\mathrm{t}, J=7.3 \mathrm{~Hz}, 3 \mathrm{H}), 1.27(\mathrm{~m}, 2 \mathrm{H}), 1.40(\mathrm{~m}, 2 \mathrm{H}), 1.73(\mathrm{~d}, J=7.2 \mathrm{~Hz}, 3 \mathrm{H}), 3.09(\mathrm{~m}, 2 \mathrm{H})$, $5.42(\mathrm{q}, J=7.2 \mathrm{~Hz}, 1 \mathrm{H}), 7.35(\mathrm{~m}, 1 \mathrm{H}), 7.89(\mathrm{dt}, J=1.7,7.7 \mathrm{~Hz}, 1 \mathrm{H}), 8.03(\mathrm{~d}, J=7.9 \mathrm{~Hz}, 1 \mathrm{H}), 8.37(\mathrm{br}$ $\mathrm{s}, 1 \mathrm{H}), 8.61(\mathrm{~m}, 1 \mathrm{H}), 8.62(\mathrm{~s}, 1 \mathrm{H}) ;{ }^{13} \mathrm{C}-\mathrm{NMR}\left(\mathrm{DMSO}-d_{6}\right) \delta 13.6,18.0,19.4,30.9,38.5,58.5,119.4,122.4$, 123.0, 137.2, 147.0, 149.6, 150.0, 168.2; LC-MS (ESI) $\mathrm{m} / \mathrm{z} 274\left([\mathrm{M}+1]^{+}\right)$.

N-Butyl-2-(4-butyl-1H-1,2,3-triazol-1-yl)propanamide (1ebf). White solid; Yield: $81 \% . \quad{ }^{1} \mathrm{H}-\mathrm{NMR}$ $\left(\right.$ DMSO- $\left.d_{6}\right) \delta 0.85(\mathrm{t}, J=7.3 \mathrm{~Hz}, 3 \mathrm{H}), 0.89(\mathrm{t}, J=7.4 \mathrm{~Hz}, 3 \mathrm{H}), 1.26(\mathrm{~m}, 2 \mathrm{H}), 1.32(\mathrm{~m}, 2 \mathrm{H}), 1.37(\mathrm{~m}, 2 \mathrm{H})$, $1.59(\mathrm{~m}, 2 \mathrm{H}), 1.61(\mathrm{~d}, J=7.2 \mathrm{~Hz}, 3 \mathrm{H}), 2.60(\mathrm{~d}, J=7.6 \mathrm{~Hz}, 2 \mathrm{H}), 3.06(\mathrm{~m}, 2 \mathrm{H}), 5.27(\mathrm{~d}, J=7.2 \mathrm{~Hz}, 1 \mathrm{H})$, $7.88(\mathrm{~s}, 1 \mathrm{H}), 8.29$ (br s, $1 \mathrm{H}) ;{ }^{13} \mathrm{C}-\mathrm{NMR}$ (DMSO- $d_{6}$ ) $\delta$ 13.6, 13.7, 18.0, 19.4, 21.7, 24.7, 30.9, 31.1, 38.4, 58.0, 120.7, 146.6, 168.4; LC-MS (ESI) $m / z 253\left([\mathrm{M}+1]^{+}\right)$.

N-Isopropyl-2-(4-phenyl-1H-1,2,3-triazol-1-yl)propanamide (1fba). White solid; Yield: $88 \%$. ${ }^{1} \mathrm{H}-\mathrm{NMR}$ $\left(\right.$ DMSO- $\left.d_{6}\right) \delta 1.07(\mathrm{~d}, J=6.6 \mathrm{~Hz}, 3 \mathrm{H}), 1.09(\mathrm{~d}, J=6.6 \mathrm{~Hz}, 3 \mathrm{H}), 1.70(\mathrm{~d}, J=7.2 \mathrm{~Hz}, 3 \mathrm{H}), 3.81(\mathrm{~m}, 1 \mathrm{H}), 5.35$ $(\mathrm{q}, J=7.1 \mathrm{~Hz}, 1 \mathrm{H}), 7.33(\mathrm{~m}, 1 \mathrm{H}), 7.45(\mathrm{t}, J=7.7 \mathrm{~Hz}, 2 \mathrm{H}), 7.88(\mathrm{~d}, J=7.1 \mathrm{~Hz}, 1 \mathrm{H}), 8.33(\mathrm{~d}, J=7.5 \mathrm{~Hz}, 1 \mathrm{H})$, $8.68(\mathrm{~s}, 1 \mathrm{H}) ;{ }^{13} \mathrm{C}-\mathrm{NMR}\left(\mathrm{DMSO}-d_{6}\right) \delta 18.0,22.1,40.9,58.3,120.6,125.1,127.8,128.9,130.9,146.0,167.5$; LC-MS (ESI) $m / z 259\left([\mathrm{M}+1]^{+}\right)$.

N-Isopropyl-2-[4-(4-methoxyphenyl)-1H-1,2,3-triazol-1-yl]propanamide (1fbb). Light brown solid; Yield: 83\%. ${ }^{1} \mathrm{H}-\mathrm{NMR}\left(\mathrm{DMSO}-\mathrm{d}_{6}\right) \delta 1.06(\mathrm{~d}, J=6.6 \mathrm{~Hz}, 3 \mathrm{H}), 1.09(\mathrm{~d}, J=6.6 \mathrm{~Hz}, 3 \mathrm{H}), 1.69(\mathrm{~d}, J=7.2 \mathrm{~Hz}, 3 \mathrm{H})$, $3.79(\mathrm{~s}, 3 \mathrm{H}), 3.82(\mathrm{~m}, 1 \mathrm{H}), 5.33(\mathrm{q}, J=7.2 \mathrm{~Hz}, 1 \mathrm{H}), 7.01(\mathrm{~d}, J=8.8 \mathrm{~Hz}, 2 \mathrm{H}), 7.80(\mathrm{~d}, J=8.8 \mathrm{~Hz}, 2 \mathrm{H}), 8.32$ $(\mathrm{d}, J=7.5 \mathrm{~Hz}, 1 \mathrm{H}), 8.56(\mathrm{~s}, 1 \mathrm{H}) ;{ }^{13} \mathrm{C}-\mathrm{NMR}\left(\mathrm{DMSO}-d_{6}\right) \delta 18.0,22.1,40.9,55.1,58.3,114.3,119.6,123.5$, 126.4, 145.9, 158.9, 167.5; LC-MS (ESI) $m / z 289\left([\mathrm{M}+1]^{+}\right)$.

2-[4-(4-Cyanophenyl)-1H-1,2,3-triazol-1-yl]-N-isopropyl-propanamide (1fbc). White solid; Yield: 78\%. $\left.{ }^{1} \mathrm{H}-\mathrm{NMR}(\text { DMSO-d })_{6}\right) \delta 1.07(\mathrm{~d}, J=6.6 \mathrm{~Hz}, 3 \mathrm{H}), 1.09(\mathrm{~d}, J=6.6 \mathrm{~Hz}, 3 \mathrm{H}), 1.71(\mathrm{~d}, J=7.3 \mathrm{~Hz}, 3 \mathrm{H})$, $3.80(\mathrm{~m}, 1 \mathrm{H}), 5.38(\mathrm{q}, J=7.2 \mathrm{~Hz}, 1 \mathrm{H}), 7.92(\mathrm{~d}, J=8.4 \mathrm{~Hz}, 2 \mathrm{H}), 8.09(\mathrm{~d}, J=8.4 \mathrm{~Hz}, 2 \mathrm{H}), 8.36(\mathrm{~d}, J=7.3 \mathrm{~Hz}$, $1 \mathrm{H}), 8.93(\mathrm{~s}, 1 \mathrm{H}) ;{ }^{13} \mathrm{C}-\mathrm{NMR}\left(\mathrm{DMSO}-d_{6}\right) \delta 18.0,22.1,40.9,58.4,110.0,118.8,122.5,125.6,133.0,135.3$, 144.5, 167.3; LC-MS (ESI) $m / z 284\left([\mathrm{M}+1]^{+}\right)$.

N-Isopropyl-2-[4-(thiophen-3-yl)-1H-1,2,3-triazol-1-yl]propanamide (1fbd). White solid; Yield: 75\%. ${ }^{1} \mathrm{H}-\mathrm{NMR}\left(\right.$ DMSO- $\left.d_{6}\right) \delta 1.06(\mathrm{~d}, J=6.6 \mathrm{~Hz}, 3 \mathrm{H}), 1.09(\mathrm{~d}, J=6.6 \mathrm{~Hz}, 3 \mathrm{H}), 1.68(\mathrm{~d}, J=7.2 \mathrm{~Hz}, 3 \mathrm{H})$, $3.81(\mathrm{~m}, 1 \mathrm{H}), 5.34(\mathrm{q}, J=7.1 \mathrm{~Hz}, 1 \mathrm{H}), 7.55(\mathrm{dd}, J=1.2,5.0 \mathrm{~Hz}, 1 \mathrm{H}), 7.64(\mathrm{dd}, J=2.9,5.0 \mathrm{~Hz}, 1 \mathrm{H}), 7.86$ 
$(\mathrm{dd}, J=1.2,3.0 \mathrm{~Hz}, 1 \mathrm{H}), 8.33(\mathrm{~d}, J=7.4 \mathrm{~Hz}, 1 \mathrm{H}), 8.55(\mathrm{~s}, 1 \mathrm{H}) ;{ }^{13} \mathrm{C}-\mathrm{NMR}\left(\mathrm{DMSO}-d_{6}\right) \delta 18.1,22.1,40.9$, $58.2,120.3,120.6,125.8,127.0,132.2,142.6,167.5$; LC-MS (ESI) $m / z 265\left([\mathrm{M}+1]^{+}\right)$.

N-Isopropyl-2-[4-(pyridin-2-yl)-1H-1,2,3-triazol-1-yl]propanamide (1fbe). White solid; Yield: 81\%. ${ }^{1} \mathrm{H}-\mathrm{NMR}\left(\right.$ DMSO- $\left.d_{6}\right) \delta 1.06(\mathrm{~d}, J=6.6 \mathrm{~Hz}, 3 \mathrm{H}), 1.09(\mathrm{~d}, J=6.6 \mathrm{~Hz}, 3 \mathrm{H}), 1.72(\mathrm{~d}, J=7.2 \mathrm{~Hz}, 3 \mathrm{H})$, $3.82(\mathrm{~m}, 1 \mathrm{H}), 5.38(\mathrm{q}, J=7.2 \mathrm{~Hz}, 1 \mathrm{H}), 7.35(\mathrm{ddd}, J=1.2,4.8,7.5 \mathrm{~Hz}, 1 \mathrm{H}), 7.89(\mathrm{dt}, J=1.8,7.7 \mathrm{~Hz}, 1 \mathrm{H})$, $8.31(\mathrm{~d}, J=7.5 \mathrm{~Hz}, 1 \mathrm{H}), 8.60(\mathrm{~m}, 1 \mathrm{H}), 8.61(\mathrm{~s}, 1 \mathrm{H}) ;{ }^{13} \mathrm{C}-\mathrm{NMR}\left(\mathrm{DMSO}-d_{6}\right) \delta 18.0,22.1,40.9,58.4,119.4$, $122.3,122.9,137.2,146.9,149.6,150.0,167.3$; LC-MS (ESI) $m / z 260\left([\mathrm{M}+1]^{+}\right)$.

2-(4-Butyl-1H-1,2,3-triazol-1-yl)- N-isopropyl-propanamide (1fbf). White solid; Yield: $82 \% .{ }^{1} \mathrm{H}-\mathrm{NMR}$ $\left(\mathrm{DMSO}-d_{6}\right) \delta 0.89(\mathrm{t}, J=7.43 \mathrm{~Hz}, 3 \mathrm{H}), 1.04(\mathrm{~d}, J=6.6 \mathrm{~Hz}, 3 \mathrm{H}), 1.07(\mathrm{~d}, J=6.6 \mathrm{~Hz}, 3 \mathrm{H}), 1.32(\mathrm{~m}, 2 \mathrm{H}), 1.57$ $(\mathrm{m}, 2 \mathrm{H}), 1.61(\mathrm{~d}, J=7.2 \mathrm{~Hz}, 3 \mathrm{H}), 2.60(\mathrm{t}, J=7.6 \mathrm{~Hz}, 2 \mathrm{H}), 3.79(\mathrm{~m}, 1 \mathrm{H}), 5.24(\mathrm{q}, J=7.2 \mathrm{~Hz}, 1 \mathrm{H}), 7.88(\mathrm{~s}$, $1 \mathrm{H}), 8.24(\mathrm{~d}, J=7.4 \mathrm{~Hz}, 1 \mathrm{H}) ;{ }^{13} \mathrm{C}-\mathrm{NMR}\left(\mathrm{DMSO}_{-} \mathrm{d}_{6}\right) \delta 13.7,18.1,21.7,22.1,24.7,31.1,40.8,58.0,120.7$, 146.5, 167.6; LC-MS (ESI) $m / z 239\left([\mathrm{M}+1]^{+}\right)$.

\subsection{General Procedure for the Preparation of Tertiary 1,2,3-Triazoloamides 2 in Solution-Phase}

A typical procedure for the desired tertiary 1,2,3-triazoloamide 2, as exemplified for 1-morpholino-2-(4-phenyl-1H-1,2,3-triazol-1-yl)ethanone (2aaa; $\mathrm{R}^{1} \mathrm{R}^{2} \mathrm{~N}=$ morpholine, $\mathrm{A}=\mathrm{CH}_{2}$, $\mathrm{R}^{3}=\mathrm{Ph}$ ).

\subsubsection{Preparation of Chloro-Amide 15}

To a solution of morpholine $(\mathbf{1 4 a} ; 4.50 \mathrm{~mL}, 51.45 \mathrm{mmol})$ and triethylamine $(7.90 \mathrm{~mL}, 56.68 \mathrm{mmol})$ in $\mathrm{CH}_{2} \mathrm{Cl}_{2}(80 \mathrm{~mL})$ was slowly added 2-chloroacetyl chloride $(6 \mathbf{6} ; 3.70 \mathrm{~mL}, 46.45 \mathrm{mmol})$ at $0{ }^{\circ} \mathrm{C}$. The reaction mixture was stirred at room temperature for $6 \mathrm{~h}$, and then diluted with $\mathrm{CH}_{2} \mathrm{Cl}_{2}$, washed with saturated $\mathrm{NaHCO}_{3}$ and brine, dried over $\mathrm{MgSO}_{4}$ and filtered. The residue was concentrated under reduced pressure to afford $\alpha$-chloroamide 15 aa $(7.55 \mathrm{~g}, 99 \%)$ as a light yellow liquid: ${ }^{1} \mathrm{H}-\mathrm{NMR}$ $\left(\mathrm{CDCl}_{3}\right) \delta 3.54(\mathrm{t}, J=4.5 \mathrm{~Hz}, 2 \mathrm{H}), 3.64(\mathrm{t}, J=4.4 \mathrm{~Hz}, 2 \mathrm{H}), 3.70-3.74(\mathrm{~m}, 4 \mathrm{H}), 4.07(\mathrm{~s}, 2 \mathrm{H}) ;{ }^{13} \mathrm{C}-\mathrm{NMR}$ $\left(\mathrm{CDCl}_{3}\right) \delta 40.7,42.5,46.8,66.6,66.7,165.3$; LC-MS (ESI) $\mathrm{m} / z 164\left([\mathrm{M}+1]^{+}\right)$.

\subsubsection{Preparation of Azidoamide 16}

To a solution of $\alpha$-chloroamide 15aa $(1.41 \mathrm{~g}$, $8.62 \mathrm{mmol})$ in acetonitrile $(20 \mathrm{~mL})$ and $\mathrm{H}_{2} \mathrm{O}(1 \mathrm{~mL})$ was added sodium azide $(700 \mathrm{mg}, 10.77 \mathrm{mmol})$. The reaction mixture was stirred at room temperature for 1 day, and then diluted with EtOAc, washed with brine, dried over $\mathrm{MgSO}_{4}$ and filtered. The solvent was removed, and the residue was passed through a short plug of silica to give $\alpha$-azidoamide 16aa $(1.40 \mathrm{~g}, 99 \%)$ as a colorless oil: ${ }^{1} \mathrm{H}-\mathrm{NMR}\left(\mathrm{CDCl}_{3}\right) \delta 3.38(\mathrm{t}, J=4.8 \mathrm{~Hz}, 2 \mathrm{H}), 3.64(\mathrm{t}, J=4.4 \mathrm{~Hz}, 2 \mathrm{H})$, 3.67-3.71 (m, 4H), $3.92(\mathrm{~s}, 2 \mathrm{H}) ;{ }^{13} \mathrm{C}-\mathrm{NMR}\left(\mathrm{CDCl}_{3}\right) \delta$ 42.3, 45.6, 50.6, 66.5, 66.8, 165.9; LC-MS (ESI) $\mathrm{m} / \mathrm{z}$ $171\left([\mathrm{M}+1]^{+}\right)$.

\subsubsection{Preparation of Tertiary 1,2,3-Triazoloamide 2}

To a mixture of $\alpha$-azidoamide 16aa (34 mg, $0.20 \mathrm{mmol}$ ) and phenylacetylene $(9 \mathbf{a} ; 24 \mu \mathrm{L}$, $0.20 \mathrm{mmol})$ in $t-\mathrm{BuOH} / \mathrm{H}_{2} \mathrm{O}(2 \mathrm{~mL}, 1: 1)$ were added $0.5 \mathrm{M} \mathrm{CuSO}_{4}(0.020 \mathrm{~mL}, 0.010 \mathrm{mmol})$ and $1.0 \mathrm{M}$ sodium ascorbate $(0.020 \mathrm{~mL}, 0.020 \mathrm{mmol})$. The reaction mixture was stirred at room temperature for 1 day, and then the resulting reaction mixture was filtered. The separated solid was washed with $\mathrm{H}_{2} \mathrm{O}$ and hexanes, and triturated with hexane/EtOAc (10:1) to give the 1-morpholino-2-(4-phenyl-1H-1,2,3-triazol-1-yl)ethanone (2aaa; $52 \mathrm{mg}, 96 \%$ ) as a white solid: $\mathrm{Mp}$ 229-231 ${ }^{\circ} \mathrm{C} ;{ }^{1} \mathrm{H}-\mathrm{NMR}\left(\mathrm{CDCl}_{3}\right) \delta 3.62(\mathrm{t}, J=4.7 \mathrm{~Hz}, 2 \mathrm{H}), 3.66(\mathrm{t}, J=4.6 \mathrm{~Hz}, 2 \mathrm{H}), 3.71(\mathrm{~m}, 4 \mathrm{H}), 5.28$ $(\mathrm{s}, 2 \mathrm{H}), 7.34(\mathrm{~m}, 1 \mathrm{H}), 7.41-7.45(\mathrm{~m}, 2 \mathrm{H}), 7.84-7.86(\mathrm{~m}, 2 \mathrm{H}), 7.99(\mathrm{~s}, 1 \mathrm{H}) ;{ }^{13} \mathrm{C}-\mathrm{NMR}\left(\mathrm{CDCl}_{3}\right) \delta$ 42.7, 46.0, 51.0, 66.5, 66.7, 121.3, 125.9, 128.4, 129.0, 130.5, 148.3, 163.7; IR (ATR) $v_{\max } 3134,2984,2858,1661$, $1643,1471,1426,1236,1112,1036,768\left(\mathrm{~cm}^{-1}\right)$; LC-MS (ESI) $\mathrm{m} / z 273\left([\mathrm{M}+1]^{+}\right)$; HRMS (FAB) calcd for $\mathrm{C}_{14} \mathrm{H}_{17} \mathrm{~N}_{4} \mathrm{O}_{2}\left([\mathrm{M}+\mathrm{H}]^{+}\right)$273.1346, found 273.1342. 


\subsection{Characterization Data of Tertiary 1,2,3-Triazoloamides 2}

2-[4-(4-Methoxyphenyl)-1H-1,2,3-triazol-1-yl]-1-morpholinoethanone (2aab). White solid; Yield: 96\%. ${ }^{1} \mathrm{H}-\mathrm{NMR}\left(\mathrm{CDCl}_{3}\right) \delta 3.62(\mathrm{t}, J=4.7 \mathrm{~Hz}, 2 \mathrm{H}), 3.66(\mathrm{t}, J=4.6 \mathrm{~Hz}, 2 \mathrm{H}), 3.70(\mathrm{~m}, 4 \mathrm{H}), 3.85(\mathrm{~s}, 3 \mathrm{H}), 5.26$ $(\mathrm{s}, 2 \mathrm{H}), 6.97(\mathrm{~d}, J=8.7 \mathrm{~Hz}, 2 \mathrm{H}), 7.77(\mathrm{~d}, J=8.7 \mathrm{~Hz}, 2 \mathrm{H}), 7.89(\mathrm{~s}, 1 \mathrm{H}) ;{ }^{13} \mathrm{C}-\mathrm{NMR}\left(\mathrm{DMSO}-d_{6}\right) \delta 41.9,44.7$, $50.9,65.87,65.94,114.3,122.1,123.4,126.4,146.0,158.9,164.5$; LC-MS (ESI) $m / z 303\left([\mathrm{M}+1]^{+}\right)$.

4-[1-(2-Morpholino-2-oxoethyl)-1H-1,2,3-triazol-4-yl]benzonitrile (2aac). Yellow solid; Yield: 93\%. ${ }^{1} \mathrm{H}-\mathrm{NMR}\left(\mathrm{CDCl}_{3}\right) \delta 3.62(\mathrm{t}, J=4.8 \mathrm{~Hz}, 2 \mathrm{H}), 3.67(\mathrm{t}, J=4.8 \mathrm{~Hz}, 2 \mathrm{H}), 3.72-3.76(\mathrm{~m}, 4 \mathrm{H}), 5.31(\mathrm{~s}, 2 \mathrm{H})$, $7.72(\mathrm{~d}, J=8.3 \mathrm{~Hz}, 2 \mathrm{H}), 7.96(\mathrm{~d}, J=8.3 \mathrm{~Hz}, 2 \mathrm{H}), 8.10(\mathrm{~s}, 1 \mathrm{H}) ;{ }^{13} \mathrm{C}-\mathrm{NMR}\left(\mathrm{DMSO}-d_{6}\right) \delta$ 41.9. 44.7. 50.9. 65.86. 65.92. 110.2. 118.8. 124.8. 125.7. 133.0. 135.3. 144.6. 164.3; LC-MS (ESI) $m / z 298\left([M+1]^{+}\right)$.

1-Morpholino-2-[4-(thiophen-3-yl)-1H-1,2,3-triazol-1-yl]ethanone (2aad). Light brown solid; Yield: 92\%. ${ }^{1} \mathrm{H}-\mathrm{NMR}\left(\mathrm{CDCl}_{3}\right) \delta$ 3.60-3.62 (m, 2H), 3.65-3.66 (m, 2H), 3.69-3.71 (m, 4H), $5.26(\mathrm{~s}, 2 \mathrm{H}), 7.39(\mathrm{dd}$, $J=3.0,5.0 \mathrm{~Hz}, 1 \mathrm{H}), 7.46(\mathrm{dd}, J=1.2,5.0 \mathrm{~Hz}, 1 \mathrm{H}), 7.70(\mathrm{dd}, J=1.3,3.0 \mathrm{~Hz}, 1 \mathrm{H}), 7.88(\mathrm{~s}, 1 \mathrm{H}) ;{ }^{13} \mathrm{C}-\mathrm{NMR}$ $\left(\right.$ DMSO- $\left._{6}\right) \delta 41.9,44.7,50.6,65.86,65.93,120.6,122.8,125.8,127.1,132.1,142.7,164.4$; LC-MS (ESI) $m / z$ $279\left([\mathrm{M}+1]^{+}\right)$.

1-Morpholino-2-[4-(pyridin-2-yl)-1H-1,2,3-triazol-1-yl]ethanone (2aae). Light brown solid; Yield: 84\%. ${ }^{1} \mathrm{H}-\mathrm{NMR}\left(\mathrm{CDCl}_{3}\right) \delta 3.55(\mathrm{t}, J=4.8 \mathrm{~Hz}, 2 \mathrm{H}), 3.61(\mathrm{t}, J=4.7 \mathrm{~Hz}, 2 \mathrm{H}), 3.65-3.68(\mathrm{~m}, 4 \mathrm{H}), 4.11(\mathrm{~s}, 2 \mathrm{H}), 5.15$ $(\mathrm{s}, 2 \mathrm{H}), 7.22(\mathrm{~m}, 1 \mathrm{H}), 7.25-7.31(\mathrm{~m}, 4 \mathrm{H}), 7.37(\mathrm{~s}, 1 \mathrm{H}) ;{ }^{13} \mathrm{C}-\mathrm{NMR}\left(\mathrm{DMSO}-d_{6}\right) \delta 41.9,44.7,50.8,65.8,65.9$, $119.3,122.9,125.0,137.2,147.0,149.6,150.0,164.4$; LC-MS (ESI) $m / z 274\left([\mathrm{M}+1]^{+}\right)$.

2-[4-(3-Methoxyphenyl)-1H-1,2,3-triazol-1-yl]-1-morpholinoethanone (2aag). White solid; Yield: 94\%. ${ }^{1} \mathrm{H}-\mathrm{NMR}\left(\mathrm{CDCl}_{3}\right) \delta 3.62(\mathrm{~m}, 2 \mathrm{H}), 3.66(\mathrm{~m}, 2 \mathrm{H}), 3.69-3.71(\mathrm{~m}, 4 \mathrm{H}), 3.87(\mathrm{~s}, 3 \mathrm{H}), 5.27(\mathrm{~s}, 2 \mathrm{H}), 6.89(\mathrm{ddd}$, $J=1.1,2.6,8.1 \mathrm{~Hz}, 1 \mathrm{H}), 7.33(\mathrm{t}, J=7.9 \mathrm{~Hz}, 1 \mathrm{H}), 7.38(\mathrm{td}, J=1.3,7.6 \mathrm{~Hz}, 1 \mathrm{H}), 7.45(\mathrm{dd}, J=1.5,2.5 \mathrm{~Hz}$, 1H), 7.97 (s, 1H); ${ }^{13} \mathrm{C}-\mathrm{NMR}\left(\mathrm{DMSO}_{6}\right) \delta 41.9,44.8,50.7,55.1,65.87,65.94,110.3,113.5,117.4,123.3$, 130.0, 132.1, 146.0, 159.7, 164.4; LC-MS (ESI) $m / z 303\left([\mathrm{M}+1]^{+}\right)$.

2-[4-(2-Methoxyphenyl)-1H-1,2,3-triazol-1-yl]-1-morpholinoethanone (2aah). White solid; Yield: 93\%. ${ }^{1} \mathrm{H}-\mathrm{NMR}\left(\mathrm{CDCl}_{3}\right) \delta 3.62(\mathrm{~m}, 2 \mathrm{H}), 3.66-3.69(\mathrm{~m}, 6 \mathrm{H}), 3.94(\mathrm{~s}, 3 \mathrm{H}), 5.27(\mathrm{~s}, 2 \mathrm{H}), 6.98(\mathrm{dd}, \mathrm{J}=0.8,8.3 \mathrm{~Hz}$, $1 \mathrm{H}), 7.08(\mathrm{dt}, J=1.0,7.6 \mathrm{~Hz}, 1 \mathrm{H}), 7.32(\mathrm{ddd}, J=1.7,7.4,8.3 \mathrm{~Hz}, 1 \mathrm{H}), 8.23(\mathrm{~s}, 1 \mathrm{H}), 8.34(\mathrm{dd}, J=1.7$, $7.7 \mathrm{~Hz}, 1 \mathrm{H}$ ); ${ }^{13} \mathrm{C}-\mathrm{NMR}\left(\mathrm{DMSO}-d_{6}\right) \delta 41.9,44.7,50.6,55.4,65.8,65.9,111.5,119.1,120.6,125.6,126.4$, $128.8,141.5,155.3,164.6$; LC-MS (ESI) $m / z 303\left([\mathrm{M}+1]^{+}\right)$.

1-Morpholino-2-(4-p-tolyl-1H-1,2,3-triazol-1-yl)ethanone (2aai). White solid; Yield: 91. ${ }^{1} \mathrm{H}-\mathrm{NMR}\left(\mathrm{CDCl}_{3}\right)$ $\delta 2.38(\mathrm{~s}, 3 \mathrm{H}), 3.62(\mathrm{~m}, 2 \mathrm{H}), 3.66(\mathrm{~m}, 2 \mathrm{H}), 3.70(\mathrm{~m}, 4 \mathrm{H}), 5.26(\mathrm{~s}, 2 \mathrm{H}), 7.24(\mathrm{~d}, J=7.9 \mathrm{~Hz}, 2 \mathrm{H}), 7.73(\mathrm{~d}$, $J=8.2 \mathrm{~Hz}, 2 \mathrm{H}), 7.94(\mathrm{~s}, 1 \mathrm{H}) ;{ }^{13} \mathrm{C}-\mathrm{NMR}\left(\mathrm{DMSO}-d_{6}\right) \delta 20.8,41.9,44.7,50.7,65.86,65.93,122.6,125.0$, 128.0, 129.4, 137.1, 146.1, 164.5; LC-MS (ESI) $m / z 287\left([\mathrm{M}+1]^{+}\right)$.

1-Morpholino-2-(4-m-tolyl-1H-1,2,3-triazol-1-yl)ethanone (2aaj). Light yellow solid; Yield: $94 \%$. ${ }^{1} \mathrm{H}-\mathrm{NMR}\left(\mathrm{CDCl}_{3}\right) \delta 2.40(\mathrm{~s}, 3 \mathrm{H}), 3.62(\mathrm{~m}, 2 \mathrm{H}), 3.66(\mathrm{~m}, 2 \mathrm{H}), 3.69(\mathrm{~m}, 4 \mathrm{H}), 5.27(\mathrm{~s}, 2 \mathrm{H}), 7.16(\mathrm{~d}$, $J=7.6 \mathrm{~Hz}, 1 \mathrm{H}), 7.31(\mathrm{t}, J=7.7 \mathrm{~Hz}, 1 \mathrm{H}), 7.61(\mathrm{~d}, J=7.7 \mathrm{~Hz}, 1 \mathrm{H}), 7.70(\mathrm{~s}, 1 \mathrm{H}), 7.97(\mathrm{~s}, 1 \mathrm{H}) ;{ }^{13} \mathrm{C}-\mathrm{NMR}$ $\left(\right.$ DMSO-d $\left.{ }_{6}\right) \delta 21.1,41.9,44.8,50.7,65.88,65.94,122.3,123.0,125.7,128.4,128.8,130.7,138.0,146.2,164.4$; LC-MS (ESI) $m / z 287\left([\mathrm{M}+1]^{+}\right)$.

1-Morpholino-2-(4-o-tolyl-1H-1,2,3-triazol-1-yl)ethanone (2aak). White solid; Yield: 93\%. ${ }^{1} \mathrm{H}-\mathrm{NMR}$ $\left(\mathrm{CDCl}_{3}\right) \delta 2.49(\mathrm{~s}, 3 \mathrm{H}), 3.62(\mathrm{~m}, 2 \mathrm{H}), 3.66(\mathrm{~m}, 2 \mathrm{H}), 3.69-3.71(\mathrm{~m}, 4 \mathrm{H}), 5.30(\mathrm{~s}, 2 \mathrm{H}), 7.27-7.29(\mathrm{~m}, 3 \mathrm{H})$, $7.79(\mathrm{~m}, 1 \mathrm{H}), 7.87(\mathrm{~s}, 1 \mathrm{H}) ;{ }^{13} \mathrm{C}-\mathrm{NMR}\left(\mathrm{DMSO}-d_{6}\right) \delta 21.1,41.9,44.7,50.6,65.86,65.94,125.0,126.0,127.7$, $128.1,130.1,130.9,134.8,145.2,164.5 ;$ LC-MS (ESI) $m / z 287\left([\mathrm{M}+1]^{+}\right)$.

2-\{4-[4-(Dimethylamino)phenyl]-1H-1,2,3-triazol-1-yl\}-1-morpholinoethanone (2aal). Light green solid; Yield: 86\%. ${ }^{1} \mathrm{H}-\mathrm{NMR}\left(\mathrm{CDCl}_{3}\right) \delta 2.99(\mathrm{~s}, 6 \mathrm{H}), 3.60-3.62(\mathrm{~m}, 2 \mathrm{H}), 3.66-3.70(\mathrm{~m}, 6 \mathrm{H}), 5.24(\mathrm{~s}, 2 \mathrm{H}), 6.77(\mathrm{~d}$, $J=8.9 \mathrm{~Hz}, 2 \mathrm{H}), 7.71(\mathrm{~d}, J=8.9 \mathrm{~Hz}, 2 \mathrm{H}), 7.84(\mathrm{~s}, 1 \mathrm{H}) ;{ }^{13} \mathrm{C}-\mathrm{NMR}\left(\mathrm{DMSO}-d_{6}\right) \delta 40.5,41.9,44.8,50.6,65.87$, $65.94,112.4,118.7,121.2,126.0,146.7,150.0,164.6$; LC-MS (ESI) $m / z 316\left([\mathrm{M}+1]^{+}\right)$. 
1-Morpholino-2-[4-(4-phenoxyphenyl)-1H-1,2,3-triazol-1-yl]ethanone (2aam). Yellow solid; Yield: 99\%. ${ }^{1} \mathrm{H}-\mathrm{NMR}\left(\mathrm{CDCl}_{3}\right) \delta 3.62(\mathrm{t}, J=4.7 \mathrm{~Hz}, 2 \mathrm{H}), 3.66(\mathrm{t}, J=4.6 \mathrm{~Hz}, 2 \mathrm{H}), 3.70-3.72(\mathrm{~m}, 4 \mathrm{H}), 5.27(\mathrm{~s}, 2 \mathrm{H})$, 7.04-7.08 (m, 4H), $7.15(\mathrm{~m}, 1 \mathrm{H}), 7.34-7.38(\mathrm{~m}, 2 \mathrm{H}), 7.80(\mathrm{~d}, J=8.8 \mathrm{~Hz}, 2 \mathrm{H}), 7.93(\mathrm{~s}, 1 \mathrm{H}) ;{ }^{13} \mathrm{C}-\mathrm{NMR}$ (DMSO- $d_{6}$ ) $\delta 41.9,44.8,50.7,65.87,65.94,118.8,118.9,122.7,123.7,126.1,126.9,130.1,145.6,156.36$, 156.44, 164.4; LC-MS (ESI) $m / z 365\left([\mathrm{M}+1]^{+}\right)$.

2-[4-(2,4-Difluorophenyl)-1H-1,2,3-triazol-1-yl]-1-morpholinoethanone (2aan). White solid; Yield: 92\%. ${ }^{1} \mathrm{H}-\mathrm{NMR}\left(\mathrm{CDCl}_{3}\right)$ 8 3.60-3.62 (m, 2H), 3.65-3.67 (m, 2H), 3.70-3.73 (m, 4H), $5.29(\mathrm{~s}, 2 \mathrm{H}), 6.90(\mathrm{ddd}$, $J=2.4,8.7,11.0 \mathrm{~Hz}, 1 \mathrm{H}), 7.00(\mathrm{~m}, 1 \mathrm{H}), 8.09(\mathrm{~d}, \mathrm{~J}=3.7 \mathrm{~Hz}, 1 \mathrm{H}), 8.27(\mathrm{dt}, J=6.5,8.6 \mathrm{~Hz}, 1 \mathrm{H}) ;{ }^{13} \mathrm{C}-\mathrm{NMR}$ $\left(\right.$ DMSO- $\left.d_{6}\right) \delta 41.9,44.7,50.8,65.8,65.9,104.6\left(\mathrm{t}, J_{\mathrm{CF}}=26.0 \mathrm{~Hz}\right), 112.3\left(\mathrm{dd}, J_{\mathrm{CF}}=21.2,3.6 \mathrm{~Hz}\right), 115.3(\mathrm{dd}$, $\left.J_{\mathrm{CF}}=13.3,3.7 \mathrm{~Hz}\right), 125.2\left(\mathrm{~d}, J_{\mathrm{CF}}=11.1 \mathrm{~Hz}\right), 128.4\left(\mathrm{dd}, J_{\mathrm{CF}}=9.7,5.3 \mathrm{~Hz}\right), 138.8\left(\mathrm{~d}, J_{\mathrm{CF}}=2.6 \mathrm{~Hz}\right), 158.5$ $\left(\mathrm{dd}, J_{\mathrm{CF}}=250.1,12.6 \mathrm{~Hz}\right), 161.7\left(\mathrm{dd}, J_{\mathrm{CF}}=247.5,12.6 \mathrm{~Hz}\right), 164.4$; LC-MS $(\mathrm{ESI}) \mathrm{m} / z 309\left([\mathrm{M}+1]^{+}\right)$.

2-(4-Benzyl-1H-1,2,3-triazol-1-yl)-1-morpholinoethanone (2aao). White solid; Yield: 94\%. ${ }^{1} \mathrm{H}-\mathrm{NMR}$ $\left(\mathrm{CDCl}_{3}\right) \delta 3.60(\mathrm{t}, J=4.7 \mathrm{~Hz}, 2 \mathrm{H}), 3.66(\mathrm{t}, J=4.6 \mathrm{~Hz}, 2 \mathrm{H}), 3.70-3.72(\mathrm{~m}, 4 \mathrm{H}), 5.28(\mathrm{~s}, 2 \mathrm{H}), 7.24(\mathrm{~m}$, $1 \mathrm{H}), 7.78(\mathrm{~m}, 1 \mathrm{H}), 8.16(\mathrm{~d}, J=7.9 \mathrm{~Hz}, 1 \mathrm{H}), 8.32(\mathrm{~s}, 1 \mathrm{H}), 8.59(\mathrm{~d}, J=4.2 \mathrm{~Hz}, 1 \mathrm{H}) ;{ }^{13} \mathrm{C}-\mathrm{NMR}\left(\mathrm{DMSO}-d_{6}\right)$ $\delta 31.2,41.9,44.7,50.4,65.8,65.9,124.2,126.1,128.4,128.5,139.7,145.7,164.6$; LC-MS (ESI) $\mathrm{m} / z 287$ $\left([\mathrm{M}+1]^{+}\right)$.

2-(4-Phenyl-1H-1,2,3-triazol-1-yl)-1-(piperidin-1-yl)ethanone (2baa). Light yellow solid; Yield: 99\%. ${ }^{1} \mathrm{H}-\mathrm{NMR}\left(\mathrm{CDCl}_{3}\right) \delta 1.56-1.62(\mathrm{~m}, 4 \mathrm{H}), 1.68(\mathrm{~m}, 2 \mathrm{H}), 3.52(\mathrm{t}, J=5.5 \mathrm{~Hz}, 2 \mathrm{H}), 3.60(\mathrm{t}, J=5.6 \mathrm{~Hz}, 2 \mathrm{H}), 5.28$ (s, 2H), $7.33(\mathrm{~m}, 1 \mathrm{H}), 7.41-7.44(\mathrm{~m}, 2 \mathrm{H}), 7.84-7.86(\mathrm{~m}, 2 \mathrm{H}), 8.00(\mathrm{~s}, 1 \mathrm{H}),{ }^{13} \mathrm{C}-\mathrm{NMR}$ (DMSO- $\left.d_{6}\right) \delta 23.8$, $25.1,25.8,42.5,45.2,50.9,123.1,125.1,127.8,128.9,130.9,146.0,163.7$; LC-MS (ESI) $m / z 271\left([\mathrm{M}+1]^{+}\right)$.

2-[4-(4-Methoxyphenyl)-1H-1,2,3-triazol-1-yl]-1-(piperidin-1-yl)ethanone (2bab). White solid; Yield: 85\%. ${ }^{1} \mathrm{H}-\mathrm{NMR}\left(\mathrm{CDCl}_{3}\right) \delta 1.56-1.62(\mathrm{~m}, 4 \mathrm{H}), 1.67(\mathrm{~m}, 2 \mathrm{H}), 3.51(\mathrm{t}, J=5.5 \mathrm{~Hz}, 2 \mathrm{H}), 3.59(\mathrm{t}, J=5.5 \mathrm{~Hz}, 2 \mathrm{H}), 3.84$ $(\mathrm{s}, 3 \mathrm{H}), 5.28(\mathrm{~s}, 2 \mathrm{H}), 6.96(\mathrm{~d}, J=8.7 \mathrm{~Hz}, 2 \mathrm{H}), 7.77(\mathrm{~d}, J=8.7 \mathrm{~Hz}, 2 \mathrm{H}), 7.90(\mathrm{~s}, 1 \mathrm{H}) ;{ }^{13} \mathrm{C}-\mathrm{NMR}\left(\mathrm{DMSO}-d_{6}\right)$ $\delta$ 23.8, 25.1, 25.8, 42.5, 45.2, 50.8, 55.1, 114.3, 122.1, 123.5, 126.4, 146.0, 158.9, 163.8; LC-MS (ESI) $\mathrm{m} / z 301$ $\left([\mathrm{M}+1]^{+}\right)$.

4-\{1-[2-Oxo-2-(piperidin-1-yl)ethyl]-1H-1,2,3-triazol-4-yl\}benzonitrile (2bac). Yellow solid; Yield: 98\%. ${ }^{1} \mathrm{H}-\mathrm{NMR}\left(\mathrm{CDCl}_{3}\right) \delta 1.59-1.67(\mathrm{~m}, 4 \mathrm{H}), 1.70(\mathrm{~m}, 2 \mathrm{H}), 3.52(\mathrm{t}, J=5.5 \mathrm{~Hz}, 2 \mathrm{H}), 3.60(\mathrm{t}, J=5.6 \mathrm{~Hz}, 2 \mathrm{H})$, $5.30(\mathrm{~s}, 2 \mathrm{H}), 7.72(\mathrm{~d}, J=8.3 \mathrm{~Hz}, 2 \mathrm{H}), 7.96(\mathrm{~d}, J=8.3 \mathrm{~Hz}, 2 \mathrm{H}), 8.10(\mathrm{~s}, 1 \mathrm{H}) ;{ }^{13} \mathrm{C}-\mathrm{NMR}\left(\right.$ DMSO- $\left.d_{6}\right) \delta$ 23.8, 25.1, 25.8, 42.5, 45.2, 51.0, 110.0, 118.8, 124.8, 125.6, 133.0, 135.3, 144.5, 163.6; LC-MS (ESI) $\mathrm{m} / z 296$ $\left([\mathrm{M}+1]^{+}\right)$.

1-(Piperidin-1-yl)-2-[4-(thiophen-3-yl)-1H-1,2,3-triazol-1-yl]ethanone (2bad). Light brown solid; Yield: 99\%. ${ }^{1} \mathrm{H}-\mathrm{NMR}\left(\mathrm{CDCl}_{3}\right) \delta 1.57-1.62(\mathrm{~m}, 4 \mathrm{H}), 1.65-1.69(\mathrm{~m}, 2 \mathrm{H}), 3.51(\mathrm{t}, J=5.5 \mathrm{~Hz}, 2 \mathrm{H}), 3.59(\mathrm{t}$, $J=5.6 \mathrm{~Hz}, 2 \mathrm{H}), 5.26(\mathrm{~s}, 2 \mathrm{H}), 7.38(\mathrm{dd}, J=3.0,5.0 \mathrm{~Hz}, 1 \mathrm{H}), 7.46(\mathrm{dd}, J=0.9,5.0 \mathrm{~Hz}, 1 \mathrm{H}), 7.69(\mathrm{dd}$, $J=1.0,2.9 \mathrm{~Hz}, 1 \mathrm{H}), 7.89(\mathrm{~s}, 1 \mathrm{H}) ;{ }^{13} \mathrm{C}-\mathrm{NMR}\left(\mathrm{DMSO}-d_{6}\right) \delta 23.8,25.1,25.8,42.5,45.2,50.8,120.6,122.9$, 125.8, 127.1, 132.2, 142.6, 163.4; LC-MS (ESI) $\mathrm{m} / z 277\left([\mathrm{M}+1]^{+}\right)$.

1-(Piperidin-1-yl)-2-[4-(pyridin-2-yl)-1H-1,2,3-triazol-1-yl]ethanone (2bae). White solid; Yield: 90\%. ${ }^{1} \mathrm{H}-\mathrm{NMR}\left(\mathrm{CDCl}_{3}\right) \delta 1.52-1.58(\mathrm{~m}, 4 \mathrm{H}), 1.61-1.66(\mathrm{~m}, 2 \mathrm{H}), 3.45(\mathrm{t}, J=5.5 \mathrm{~Hz}, 2 \mathrm{H}), 3.54(\mathrm{t}, J=5.6 \mathrm{~Hz}, 2 \mathrm{H})$, $5.26(\mathrm{~s}, 2 \mathrm{H}), 7.19(\mathrm{ddd}, J=1.2,4.9,7.5 \mathrm{~Hz}, 1 \mathrm{H}), 7.74(\mathrm{dt}, J=1.8,7.8 \mathrm{~Hz}, 1 \mathrm{H}), 8.11(\mathrm{td}, J=1.0,7.9 \mathrm{~Hz}$, $1 \mathrm{H}), 8.29(\mathrm{~s}, 1 \mathrm{H}), 8.56(\mathrm{ddd}, J=0.9,1.7,4.9 \mathrm{~Hz}, 1 \mathrm{H}) ;{ }^{13} \mathrm{C}-\mathrm{NMR}\left(\mathrm{DMSO}-d_{6}\right) \delta 23.8,25.1,25.8,42.5,45.2$, $50.9,119.3,122.8,125.0,137.2,146.9,149.6,150.1,163.7$; LC-MS (ESI) $m / z 272\left([\mathrm{M}+1]^{+}\right)$.

2-[4-(3-Methoxyphenyl)-1H-1,2,3-triazol-1-yl]-1-(piperidin-1-yl)ethanone (2bag). White solid; Yield: 93\%. ${ }^{1} \mathrm{H}-\mathrm{NMR}\left(\mathrm{CDCl}_{3}\right) \delta 1.57-1.62(\mathrm{~m}, 4 \mathrm{H}), 1.67(\mathrm{~m}, 2 \mathrm{H}), 3.52(\mathrm{t}, J=5.5 \mathrm{~Hz}, 2 \mathrm{H}), 3.60(\mathrm{t}, J=5.6 \mathrm{~Hz}, 2 \mathrm{H}), 3.87$ $(\mathrm{s}, 3 \mathrm{H}), 5.27(\mathrm{~s}, 2 \mathrm{H}), 6.89(\mathrm{ddd}, J=1.0,2.6,8.1 \mathrm{~Hz}, 1 \mathrm{H}), 7.32(\mathrm{t}, J=7.9 \mathrm{~Hz}, 1 \mathrm{H}), 7.38(\mathrm{td}, J=1.3,7.6 \mathrm{~Hz}$, $1 \mathrm{H}), 7.46(\mathrm{dd}, J=1.5,2.5 \mathrm{~Hz}, 1 \mathrm{H}), 7.99(\mathrm{~s}, 1 \mathrm{H}) ;{ }^{13} \mathrm{C}-\mathrm{NMR}\left(\mathrm{DMSO}_{6}\right) \delta$ 23.8, 25.1, 25.8, 42.5, 45.2, 50.9, 55.1, 110.2, 113.5, 117.4, 123.4, 130.0, 132.2, 145.9, 159.7, 163.7; LC-MS (ESI) $m / z 301\left([\mathrm{M}+1]^{+}\right)$. 
2-[4-(2-Methoxyphenyl)-1H-1,2,3-triazol-1-yl]-1-(piperidin-1-yl)ethanone (2bah). White solid; Yield: 95\%. ${ }^{1} \mathrm{H}-\mathrm{NMR}\left(\mathrm{CDCl}_{3}\right) \delta 1.52-1.58(\mathrm{~m}, 4 \mathrm{H}), 1.65(\mathrm{~m}, 2 \mathrm{H}), 3.50(\mathrm{t}, J=5.9 \mathrm{~Hz}, 2 \mathrm{H}), 3.58(\mathrm{t}, J=5.5 \mathrm{~Hz}, 2 \mathrm{H})$, $3.92(\mathrm{~s}, 3 \mathrm{H}), 5.26(\mathrm{~s}, 2 \mathrm{H}), 6.97(\mathrm{dd}, J=0.6,8.3 \mathrm{~Hz}, 1 \mathrm{H}), 7.07(\mathrm{dt}, J=1.0,7.6 \mathrm{~Hz}, 1 \mathrm{H}), 7.30(\mathrm{ddd}$, $J=1.7,7.4,8.3 \mathrm{~Hz}, 1 \mathrm{H}), 8.23(\mathrm{~s}, 1 \mathrm{H}), 8.34(\mathrm{dd}, J=1.7,7.7 \mathrm{~Hz}, 1 \mathrm{H}) ;{ }^{13} \mathrm{C}-\mathrm{NMR}$ (DMSO- $\left.d_{6}\right) \delta 23.8,25.1$, $25.8,42.5,45.2,50.7,22.4,111.5,119.2,120.6,125.6,126.4,128.7,141.4,155.3,163.9$; LC-MS (ESI) $m / z 301$ $\left([\mathrm{M}+1]^{+}\right)$.

1-(Piperidin-1-yl)-2-(4-p-tolyl-1H-1,2,3-triazol-1-yl)ethanone (2bai). Light yellow solid; Yield: 91\%. ${ }^{1} \mathrm{H}-\mathrm{NMR}\left(\mathrm{CDCl}_{3}\right) \delta 1.57-1.60(\mathrm{~m}, 4 \mathrm{H}), 1.67(\mathrm{~m}, 2 \mathrm{H}), 2.38(\mathrm{~s}, 3 \mathrm{H}), 3.52(\mathrm{t}, J=5.5 \mathrm{~Hz}, 2 \mathrm{H}), 3.59(\mathrm{t}$, $J=5.6 \mathrm{~Hz}, 2 \mathrm{H}), 5.26(\mathrm{~s}, 2 \mathrm{H}), 7.23(\mathrm{~d}, J=7.9 \mathrm{~Hz}, 2 \mathrm{H}), 7.73(\mathrm{~d}, J=8.2 \mathrm{~Hz}, 2 \mathrm{H}), 7.95(\mathrm{~s}, 1 \mathrm{H}),{ }^{13} \mathrm{C}-\mathrm{NMR}$ $\left(\right.$ DMSO- $_{6}$ ) $\delta 20.8,23.8,25.1,25.8,42.5,45.2,50.8,122.7,125.0,128.1,129.4,137.0,146.1,163.8$; LC-MS (ESI) $m / z 285\left([\mathrm{M}+1]^{+}\right)$.

1-(Piperidin-1-yl)-2-(4-m-tolyl-1H-1,2,3-triazol-1-yl)ethanone (2baj). Light yellow solid; Yield: 99\%. ${ }^{1} \mathrm{H}-\mathrm{NMR}\left(\mathrm{CDCl}_{3}\right) \delta 1.56-1.62(\mathrm{~m}, 4 \mathrm{H}), 1.67(\mathrm{~m}, 2 \mathrm{H}), 2.40(\mathrm{~s}, 3 \mathrm{H}), 3.51(\mathrm{t}, J=5.5 \mathrm{~Hz}, 2 \mathrm{H}), 3.59(\mathrm{t}$, $J=5.6 \mathrm{~Hz}, 2 \mathrm{H}), 5.26(\mathrm{~s}, 2 \mathrm{H}), 7.15(\mathrm{~d}, J=7.6 \mathrm{~Hz}, 1 \mathrm{H}), 7.31(\mathrm{t}, J=7.6 \mathrm{~Hz}, 1 \mathrm{H}), 7.61(\mathrm{~d}, J=7.7 \mathrm{~Hz}, 1 \mathrm{H}), 7.70$ (s, 1H), $7.97(\mathrm{~s}, 1 \mathrm{H}) ;{ }^{13} \mathrm{C}-\mathrm{NMR}$ (DMSO- $d_{6}$ ) $\delta 21.1,23.8,25.1,25.8,42.5,45.2,50.8,122.3,123.0,125.6$, 128.4, 128.8, 130.8, 138.0, 146.1, 163.7; LC-MS (ESI) $m / z 285\left([\mathrm{M}+1]^{+}\right)$.

1-(Piperidin-1-yl)-2-(4-o-tolyl-1H-1,2,3-triazol-1-yl)ethanone (2bak). Light yellow solid; Yield: 91\%. ${ }^{1} \mathrm{H}-\mathrm{NMR}\left(\mathrm{CDCl}_{3}\right) \delta 1.57-1.61(\mathrm{~m}, 4 \mathrm{H}), 1.68(\mathrm{~m}, 2 \mathrm{H}), 2.49(\mathrm{~s}, 3 \mathrm{H}), 3.53(\mathrm{t}, J=5.5 \mathrm{~Hz}, 2 \mathrm{H}), 3.60(\mathrm{t}$, $J=5.6 \mathrm{~Hz}, 2 \mathrm{H}), 5.29(\mathrm{~s}, 2 \mathrm{H}), 7.27-7.28(\mathrm{~m}, 3 \mathrm{H}), 7.79(\mathrm{~m}, 1 \mathrm{H}), 7.88(\mathrm{~s}, 1 \mathrm{H}) ;{ }^{13} \mathrm{C}-\mathrm{NMR}\left(\mathrm{DMSO}-d_{6}\right) \delta 21.1$, 23.8, 25.1, 25.8, 42.5, 45.2, 50.8, 125.0, 126.0, 127.7, 128.1, 130.1, 130.9, 134.8, 145.2, 163.8; LC-MS (ESI) $m / z 285\left([\mathrm{M}+1]^{+}\right)$.

2-\{4-[4-(Dimethylamino)phenyl]-1H-1,2,3-triazol-1-yl\}-1-(piperidin-1-yl)ethanone (2bal). Light green solid; Yield: 89\%. ${ }^{1} \mathrm{H}-\mathrm{NMR}\left(\mathrm{CDCl}_{3}\right) \delta 1.53-1.57(\mathrm{~m}, 4 \mathrm{H}), 1.64-1.68(\mathrm{~m}, 2 \mathrm{H}), 2.99(\mathrm{~s}, 6 \mathrm{H}), 3.51(\mathrm{t}$, $J=5.5 \mathrm{~Hz}, 2 \mathrm{H}), 3.58(\mathrm{t}, J=5.5 \mathrm{~Hz}, 2 \mathrm{H}), 5.24(\mathrm{~s}, 2 \mathrm{H}), 6.77(\mathrm{~d}, J=8.9 \mathrm{~Hz}, 2 \mathrm{H}), 7.71(\mathrm{~d}, J=8.9 \mathrm{~Hz}$, 2H), $7.84(\mathrm{~s}, 1 \mathrm{H}) ;{ }^{13} \mathrm{C}-\mathrm{NMR}$ (DMSO- $\left.d_{6}\right) \delta 23.8,25.1,25.8,40.0,42.5,45.2,50.7,112.4,118.8,121.2,126.0$, 146.6, 149.9, 163.9; LC-MS (ESI) $m / z 314\left([\mathrm{M}+1]^{+}\right)$.

2-[4-(4-Phenoxyphenyl)-1H-1,2,3-triazol-1-yl]-1-(piperidin-1-yl)ethanone (2bam). Light yellow solid; Yield: $99 \% .{ }^{1} \mathrm{H}-\mathrm{NMR}\left(\mathrm{CDCl}_{3}\right) \delta 1.59-1.62(\mathrm{~m}, 4 \mathrm{H}), 1.66-1.71(\mathrm{~m}, 2 \mathrm{H}), 3.52(\mathrm{t}, J=5.5 \mathrm{~Hz}, 2 \mathrm{H}), 3.59$ $(\mathrm{t}, J=5.5 \mathrm{~Hz}, 2 \mathrm{H}), 5.27(\mathrm{~s}, 2 \mathrm{H}), 7.04-7.07(\mathrm{~m}, 4 \mathrm{H}), 7.13(\mathrm{~m}, 1 \mathrm{H}), 7.34-7.37(\mathrm{~m}, 2 \mathrm{H}), 7.81(\mathrm{~d}, J=8.8 \mathrm{~Hz}$, 2H), $7.94(\mathrm{~s}, 1 \mathrm{H}) ;{ }^{13} \mathrm{C}-\mathrm{NMR}$ (DMSO- $d_{6}$ ) $\delta 23.8,25.1,25.8,42.5,45.2,50.8,118.8,118.9,122.7,123.6$, 126.2, 126.8, 130.1, 145.6, 156.3, 156.4, 163.7; LC-MS (ESI) $\mathrm{m} / z 363\left([\mathrm{M}+1]^{+}\right)$.

2-[4-(2,4-Difluorophenyl)-1H-1,2,3-triazol-1-yl]-1-(piperidin-1-yl)ethanone (2ban). Yellow solid; Yield: 99\%. ${ }^{1} \mathrm{H}-\mathrm{NMR}\left(\mathrm{CDCl}_{3}\right) \delta 1.58-1.64(\mathrm{~m}, 4 \mathrm{H}), 1.66-1.71(\mathrm{~m}, 2 \mathrm{H}), 3.51(\mathrm{t}, J=5.5 \mathrm{~Hz}, 2 \mathrm{H}), 3.60(\mathrm{t}$, $J=5.6 \mathrm{~Hz}, 2 \mathrm{H}), 5.29(\mathrm{~s}, 2 \mathrm{H}), 6.90(\mathrm{ddd}, J=2.4,8.7,11.0 \mathrm{~Hz}, 1 \mathrm{H}), 6.99(\mathrm{~m}, 1 \mathrm{H}), 8.09(\mathrm{~d}, J=3.7 \mathrm{~Hz}$, $1 \mathrm{H}), 8.27(\mathrm{dt}, J=6.5,8.6 \mathrm{~Hz}, 1 \mathrm{H}) ;{ }^{13} \mathrm{C}-\mathrm{NMR}\left(\mathrm{DMSO}-d_{6}\right) \delta 23.8,25.1,25.7,42.5,45.2,50.9,104.6(\mathrm{t}$, $\left.J_{\mathrm{CF}}=26.0 \mathrm{~Hz}\right), 112.3\left(\mathrm{~d}, J_{\mathrm{CF}}=24.6 \mathrm{~Hz}\right), 115.3\left(\mathrm{dd}, J_{\mathrm{CF}}=13.1,4.0 \mathrm{~Hz}\right), 125.2\left(\mathrm{~d}, J_{\mathrm{CF}}=11.9 \mathrm{~Hz}\right), 128.4$ $\left(\mathrm{dd}, J_{\mathrm{CF}}=9.4,5.7 \mathrm{~Hz}\right), 138.7\left(\mathrm{~d}, J_{\mathrm{CF}}=2.4 \mathrm{~Hz}\right), 158.5\left(\mathrm{dd}, J_{\mathrm{CF}}=250.0,12.6 \mathrm{~Hz}\right), 161.6\left(\mathrm{dd}, J_{\mathrm{CF}}=247.5\right.$, $12.6 \mathrm{~Hz}), 163.7$; LC-MS (ESI) $m / z 307\left([\mathrm{M}+1]^{+}\right)$.

2-(4-Benzyl-1H-1,2,3-triazol-1-yl)-1-(piperidin-1-yl)ethanone (2bao). White solid; Yield: 95\%. ${ }^{1} \mathrm{H}-\mathrm{NMR}$ $\left(\mathrm{CDCl}_{3}\right) \delta 1.52-1.57(\mathrm{~m}, 4 \mathrm{H}), 1.63-1.67(\mathrm{~m}, 2 \mathrm{H}), 3.45(\mathrm{t}, J=5.5 \mathrm{~Hz}, 2 \mathrm{H}), 3.56(\mathrm{t}, J=5.6 \mathrm{~Hz}, 2 \mathrm{H}), 4.10(\mathrm{~s}$, 2H), $5.15(\mathrm{~s}, 2 \mathrm{H}), 7.20(\mathrm{~m}, 1 \mathrm{H}), 7.24-7.30(\mathrm{~m}, 4 \mathrm{H}), 7.37(\mathrm{~s}, 1 \mathrm{H}) ;{ }^{13} \mathrm{C}-\mathrm{NMR}$ (DMSO- $\left.d_{6}\right) \delta 23.8,25.1,25.7$, 31.2, 42.4, 45.2, 50.5, 124.2, 126.1, 128.4, 128.5, 139.7, 145.6, 163.9; LC-MS (ESI) $\mathrm{m} / \mathrm{z} 285\left([\mathrm{M}+1]^{+}\right)$.

2-(4-Phenyl-1H-1,2,3-triazol-1-yl)-1-(pyrrolidin-1-yl)ethanone (2caa). Light yellow solid; Yield: 94\%. ${ }^{1} \mathrm{H}-\mathrm{NMR}\left(\mathrm{CDCl}_{3}\right) \delta 1.92(\mathrm{tt}, J=6.7,7.0 \mathrm{~Hz}, 2 \mathrm{H}), 2.05(\mathrm{tt}, J=6.8,7.0 \mathrm{~Hz}, 2 \mathrm{H}), 3.54(\mathrm{t}, J=7.0 \mathrm{~Hz}$, $2 \mathrm{H}), 3.58(\mathrm{t}, J=6.9 \mathrm{~Hz}, 2 \mathrm{H}), 5.19(\mathrm{~s}, 2 \mathrm{H}), 7.33(\mathrm{~m}, 1 \mathrm{H}), 7.41-7.44(\mathrm{~m}, 2 \mathrm{H}), 7.84-7.86(\mathrm{~m}, 2 \mathrm{H}), 8.06(\mathrm{~s}$, 1H); ${ }^{13}$ C-NMR (DMSO- $d_{6}$ ) $\delta$ 23.7, 25.6, 45.1, 45.8, 51.4, 123.0, 125.1, 127.8, 128.9, 130.8, 146.0, 163.7; LC-MS (ESI) $m / z 257\left([\mathrm{M}+1]^{+}\right)$. 
2-[4-(4-Methoxyphenyl)-1H-1,2,3-triazol-1-yl]-1-(pyrrolidin-1-yl)ethanone (2cab). White solid; Yield: 89\%. ${ }^{1} \mathrm{H}-\mathrm{NMR}\left(\mathrm{CDCl}_{3}\right) \delta 1.91(\mathrm{tt}, J=6.8,7.1 \mathrm{~Hz}, 2 \mathrm{H}), 2.03(\mathrm{tt}, J=6.8,7.0 \mathrm{~Hz}, 2 \mathrm{H}), 3.53(\mathrm{t}, J=7.0 \mathrm{~Hz}, 2 \mathrm{H})$, $3.58(\mathrm{t}, J=6.9 \mathrm{~Hz}, 2 \mathrm{H}), 3.84(\mathrm{~s}, 3 \mathrm{H}), 5.17(\mathrm{~s}, 2 \mathrm{H}), 6.96(\mathrm{~d}, J=8.9 \mathrm{~Hz}, 2 \mathrm{H}), 7.77(\mathrm{~d}, J=8.9 \mathrm{~Hz}, 2 \mathrm{H}), 7.96$ $(\mathrm{s}, 1 \mathrm{H}) ;{ }^{13} \mathrm{C}-\mathrm{NMR}\left(\mathrm{DMSO}-d_{6}\right) \delta 23.7,25.6,45.1,45.8,51.4,55.1,114.3,122.0,1234,126.4,146.0,158.9$, 163.7; LC-MS (ESI) $m / z 287\left([\mathrm{M}+1]^{+}\right)$.

4-\{1-[2-Oxo-2-(pyrrolidin-1-yl)ethyl]-1H-1,2,3-triazol-4-yl\}benzonitrile (2cac). Yellow solid; Yield: 94\%. ${ }^{1} \mathrm{H}-\mathrm{NMR}\left(\mathrm{CDCl}_{3}\right) \delta 1.93(\mathrm{tt}, J=6.9,7.1 \mathrm{~Hz}, 2 \mathrm{H}), 2.07(\mathrm{tt}, J=6.8,6.9 \mathrm{~Hz}, 2 \mathrm{H}), 3.54(\mathrm{t}, J=7.0 \mathrm{~Hz}, 2 \mathrm{H})$, $3.60(\mathrm{t}, J=6.9 \mathrm{~Hz}, 2 \mathrm{H}), 5.21(\mathrm{~s}, 2 \mathrm{H}), 7.71(\mathrm{~d}, J=8.7 \mathrm{~Hz}, 2 \mathrm{H}), 7.96(\mathrm{~d}, J=8.7 \mathrm{~Hz}, 2 \mathrm{H}), 8.17(\mathrm{~s}, 1 \mathrm{H})$; ${ }^{13} \mathrm{C}$ - NMR (DMSO- $d_{6}$ ) $\delta$ 23.7, 25.6, 45.1, 45.9, 51.5, 110.0, 118.8, 124.7, 125.7, 133.0, 135.3, 144.5, 163.5; LC-MS (ESI) $m / z 282\left([\mathrm{M}+1]^{+}\right)$.

1-(Pyrrolidin-1-yl)-2-[4-(thiophen-3-yl)-1H-1,2,3-triazol-1-yl]ethanone (2cad). Light green solid; Yield: $88 \% .{ }^{1} \mathrm{H}-\mathrm{NMR}\left(\mathrm{CDCl}_{3}\right) \delta 1.91(\mathrm{tt}, J=6.7,6.9 \mathrm{~Hz}, 2 \mathrm{H}), 2.05(\mathrm{tt}, J=6.8,6.9 \mathrm{~Hz}, 2 \mathrm{H}), 3.53(\mathrm{t}, J=7.0 \mathrm{~Hz}$, $2 \mathrm{H}), 3.58(\mathrm{t}, J=6.9 \mathrm{~Hz}, 2 \mathrm{H}), 5.18(\mathrm{~s}, 2 \mathrm{H}), 7.38(\mathrm{dd}, J=3.0,5.0 \mathrm{~Hz}, 1 \mathrm{H}), 7.47(\mathrm{dd}, J=1.2,5.0 \mathrm{~Hz}, 1 \mathrm{H}), 7.69$ $(\mathrm{dd}, J=1.3,3.0 \mathrm{~Hz}, 1 \mathrm{H}), 7.96(\mathrm{~s}, 1 \mathrm{H}) ;{ }^{13} \mathrm{C}-\mathrm{NMR}\left(\mathrm{DMSO}-d_{6}\right) \delta$ 23.7, 25.6, 45.1, 45.8, 51.3, 120.6, 122.7, $125.8,127.1,132.2,142.6,163.7$; LC-MS (ESI) $\mathrm{m} / z 263\left([\mathrm{M}+1]^{+}\right)$.

2-[4-(Pyridin-2-yl)-1H-1,2,3-triazol-1-yl]-1-(pyrrolidin-1-yl)ethanone (2cae). Light yellow solid; Yield: $87 \% .{ }^{1} \mathrm{H}-\mathrm{NMR}\left(\mathrm{CDCl}_{3}\right) \delta 1.89(\mathrm{tt}, J=6.9,7.0 \mathrm{~Hz}, 2 \mathrm{H}), 2.02(\mathrm{tt}, J=6.8,6.9 \mathrm{~Hz}, 2 \mathrm{H}), 3.52(\mathrm{t}, J=6.9 \mathrm{~Hz}$, $2 \mathrm{H}), 3.54(\mathrm{t}, J=6.8 \mathrm{~Hz}, 2 \mathrm{H}), 5.20(\mathrm{~s}, 2 \mathrm{H}), 7.21(\mathrm{ddd}, J=1.2,4.9,7.5 \mathrm{~Hz}, 1 \mathrm{H}), 7.76(\mathrm{dt}, J=1.8,7.7 \mathrm{~Hz}$, $1 \mathrm{H}), 8.14(\mathrm{td}, J=1.0,7.9 \mathrm{~Hz}, 1 \mathrm{H}), 8.35(\mathrm{~s}, 1 \mathrm{H}), 8.58(\mathrm{ddd}, J=0.9,1.7,4.9 \mathrm{~Hz}, 1 \mathrm{H}) ;{ }^{13} \mathrm{C}-\mathrm{NMR}\left(\mathrm{DMSO}-d_{6}\right)$ $\delta$ 23.7, 25.6, 45.2, 45.9, 51.5, 119.4, 122.9, 124.8, 137.2, 146.9, 149.6, 150.1, 163.6; LC-MS (ESI) $\mathrm{m} / \mathrm{z} 258$ $\left([\mathrm{M}+1]^{+}\right)$.

2-[4-(3-Methoxyphenyl)-1H-1,2,3-triazol-1-yl]-1-(pyrrolidin-1-yl)ethanone (2cag). White solid; Yield: 93\%. ${ }^{1} \mathrm{H}-\mathrm{NMR}\left(\mathrm{CDCl}_{3}\right) \delta 1.92(\mathrm{tt}, J=6.8,6.8 \mathrm{~Hz}, 2 \mathrm{H}), 2.05(\mathrm{tt}, J=6.8,6.9 \mathrm{~Hz}, 2 \mathrm{H}), 3.54(\mathrm{t}, J=7.0 \mathrm{~Hz}, 2 \mathrm{H})$, $3.58(\mathrm{t}, J=6.9 \mathrm{~Hz}, 2 \mathrm{H}), 3.87(\mathrm{~s}, 3 \mathrm{H}), 5.19(\mathrm{~s}, 2 \mathrm{H}), 6.88(\mathrm{ddd}, J=1.0,2.6,8.2 \mathrm{~Hz}, 1 \mathrm{H}), 7.32(\mathrm{t}, J=7.9 \mathrm{~Hz}$, $1 \mathrm{H}), 7.39(\mathrm{td}, J=1.3,7.6 \mathrm{~Hz}, 1 \mathrm{H}), 7.46(\mathrm{dd}, J=1.6,2.5 \mathrm{~Hz}, 1 \mathrm{H}), 8.05(\mathrm{~s}, 1 \mathrm{H}) ;{ }^{13} \mathrm{C}-\mathrm{NMR}\left(\mathrm{DMSO}-d_{6}\right) \delta$ 23.7, 25.6, 45.2, 45.8, 51.4, 55.1, 110.3, 113.5, 117.5, 123.2, 130.1, 132.2, 146.0, 159.7, 163.7; LC-MS (ESI) $\mathrm{m} / \mathrm{z} 287\left([\mathrm{M}+1]^{+}\right)$.

2-[4-(2-Methoxyphenyl)-1H-1,2,3-triazol-1-yl]-1-(pyrrolidin-1-yl)ethanone (2cah). White solid; Yield: 89\%. ${ }^{1} \mathrm{H}-\mathrm{NMR}\left(\mathrm{CDCl}_{3}\right) \delta 1.90(\mathrm{tt}, J=6.8,6.9 \mathrm{~Hz}, 2 \mathrm{H}), 2.02(\mathrm{tt}, J=6.8,6.8 \mathrm{~Hz}, 2 \mathrm{H}), 3.53(\mathrm{t}, J=7.0 \mathrm{~Hz}, 2 \mathrm{H}), 3.56$ $(\mathrm{t}, J=6.9 \mathrm{~Hz}, 2 \mathrm{H}), 3.93(\mathrm{~s}, 3 \mathrm{H}), 5.19(\mathrm{~s}, 2 \mathrm{H}), 6.72(\mathrm{dd}, J=0.7,8.3 \mathrm{~Hz}, 1 \mathrm{H}), 7.07(\mathrm{dt}, J=1.0,7.5 \mathrm{~Hz}, 1 \mathrm{H})$, $7.31(\mathrm{ddd}, J=1.7,7.4,8.3 \mathrm{~Hz}, 1 \mathrm{H}), 8.29(\mathrm{~s}, 1 \mathrm{H}), 8.34(\mathrm{dd}, J=1.7,7.7 \mathrm{~Hz}, 1 \mathrm{H}) ;{ }^{13} \mathrm{C}-\mathrm{NMR}\left(\mathrm{DMSO}-d_{6}\right) \delta$ 23.7, 25.6, 45.1, 45.8, 51.2, 55.5, 111.5, 119.2, 120.7, 125.5, 126.4, 128.8, 141.5, 155.3, 163.9; LC-MS (ESI) $\mathrm{m} / \mathrm{z} 287\left([\mathrm{M}+1]^{+}\right)$.

1-(Pyrrolidin-1-yl)-2-(4-p-tolyl-1H-1,2,3-triazol-1-yl)ethanone (2cai). White solid; Yield: 96\%. ${ }^{1} \mathrm{H}-\mathrm{NMR}$ $\left(\mathrm{CDCl}_{3}\right) \delta 1.91(\mathrm{tt}, J=6.9,6.9 \mathrm{~Hz}, 2 \mathrm{H}), 2.04(\mathrm{tt}, J=6.8,6.8 \mathrm{~Hz}, 2 \mathrm{H}), 2.38(\mathrm{~s}, 3 \mathrm{H}), 3.53(\mathrm{t}, J=7.0 \mathrm{~Hz}$, $2 \mathrm{H}), 3.57(\mathrm{t}, J=6.9 \mathrm{~Hz}, 2 \mathrm{H}), 5.18(\mathrm{~s}, 2 \mathrm{H}), 7.23(\mathrm{~d}, J=8.0 \mathrm{~Hz}, 2 \mathrm{H}), 7.73(\mathrm{~d}, J=8.1 \mathrm{~Hz}, 2 \mathrm{H}), 8.01(\mathrm{~s}, 1 \mathrm{H})$; ${ }^{13}$ C-NMR (DMSO- $d_{6}$ ) $\delta 20.8,23.7,25.6,45.2,45.8$ 51.4, 122.5, 125.0, 128.1, 129.5, 137.1, 146.1, 163.7; LC-MS (ESI) $m / z 271\left([\mathrm{M}+1]^{+}\right)$.

1-(Pyrrolidin-1-yl)-2-(4-m-tolyl-1H-1,2,3-triazol-1-yl)ethanone (2caj). White solid; Yield: 96\%. ${ }^{1} \mathrm{H}-\mathrm{NMR}$ $\left(\mathrm{CDCl}_{3}\right) \delta 1.91(\mathrm{tt}, J=6.8,6.8 \mathrm{~Hz}, 2 \mathrm{H}), 2.03(\mathrm{tt}, J=6.8,6.8 \mathrm{~Hz}, 2 \mathrm{H}), 2.40(\mathrm{~s}, 3 \mathrm{H}), 3.53(\mathrm{t}, J=7.0 \mathrm{~Hz}$, $2 \mathrm{H}), 3.57(\mathrm{t}, J=6.8 \mathrm{~Hz}, 2 \mathrm{H}), 5.18(\mathrm{~s}, 2 \mathrm{H}), 7.14(\mathrm{~d}, J=7.6 \mathrm{~Hz}, 1 \mathrm{H}), 7.30(\mathrm{t}, J=7.7 \mathrm{~Hz}, 1 \mathrm{H}), 7.61(\mathrm{~d}$, $J=7.7 \mathrm{~Hz}, 1 \mathrm{H}), 7.70(\mathrm{~s}, 1 \mathrm{H}), 8.03(\mathrm{~s}, 1 \mathrm{H}) ;{ }^{13} \mathrm{C}-\mathrm{NMR}\left(\mathrm{DMSO}-d_{6}\right) \delta 21.1,23.7,25.6,45.1,45.8,51.4,122.3$, 122.9, 125.6, 128.4, 128.8, 130.7, 138.0, 146.1, 163.7; LC-MS (ESI) $m / z 271\left([\mathrm{M}+1]^{+}\right)$.

1-(Pyrrolidin-1-yl)-2-(4-o-tolyl-1H-1,2,3-triazol-1-yl)ethanone (2cak). White solid; Yield: 91\%. ${ }^{1} \mathrm{H}-\mathrm{NMR}$ $\left(\mathrm{CDCl}_{3}\right) \delta 1.92(\mathrm{tt}, J=6.8,6.9 \mathrm{~Hz}, 2 \mathrm{H}), 2.05(\mathrm{tt}, J=6.8,6.8 \mathrm{~Hz}, 2 \mathrm{H}), 2.49(\mathrm{~s}, 3 \mathrm{H}), 3.54(\mathrm{t}, J=7.0 \mathrm{~Hz}, 2 \mathrm{H})$, $3.59(\mathrm{t}, J=6.9 \mathrm{~Hz}, 2 \mathrm{H}), 5.21(\mathrm{~s}, 2 \mathrm{H}), 7.26-7.28(\mathrm{~m}, 3 \mathrm{H}), 7.79(\mathrm{~m}, 1 \mathrm{H}), 7.94(\mathrm{~s}, 1 \mathrm{H}) ;{ }^{13} \mathrm{C}-\mathrm{NMR}\left(\mathrm{DMSO}-d_{6}\right)$ 
$\delta$ 21.1, 23.7, 25.6, 45.1, 45.8, 51.3, 124.8, 126.0, 127.7, 128.1, 130.1, 130.9, 134.8, 145.2, 163.7; LC-MS (ESI) $\mathrm{m} / \mathrm{z} 271\left([\mathrm{M}+1]^{+}\right)$.

2-\{4-[4-(Dimethylamino)phenyl]-1H-1,2,3-triazol-1-yl\}-1-(pyrrolidin-1-yl)ethanone (2cal). Light yellow solid; Yield: 94\%. ${ }^{1} \mathrm{H}-\mathrm{NMR}\left(\mathrm{CDCl}_{3}\right) \delta 1.90(\mathrm{tt}, J=6.7,6.9 \mathrm{~Hz}, 2 \mathrm{H}), 2.02(\mathrm{tt}, J=6.8,6.9 \mathrm{~Hz}, 2 \mathrm{H})$, $2.99(\mathrm{~s}, 6 \mathrm{H}), 3.52(\mathrm{t}, J=7.0 \mathrm{~Hz}, 2 \mathrm{H}), 3.56(\mathrm{t}, J=6.9 \mathrm{~Hz}, 2 \mathrm{H}), 5.16(\mathrm{~s}, 2 \mathrm{H}), 6.77(\mathrm{~d}, J=9.0 \mathrm{~Hz}, 2 \mathrm{H}), 7.71(\mathrm{~d}$, $J=8.9 \mathrm{~Hz}, 2 \mathrm{H}), 7.90(\mathrm{~s}, 1 \mathrm{H}) ;{ }^{13} \mathrm{C}-\mathrm{NMR}\left(\mathrm{DMSO}_{-} d_{6}\right) \delta 23.7,25.6,40.0,45.1,45.8,51.3,112.4,118.8,121.1$, 126.0, 146.6, 150.0, 163.8; LC-MS (ESI) $m / z 300\left([\mathrm{M}+1]^{+}\right)$.

2-[4-(4-Phenoxyphenyl)-1H-1,2,3-triazol-1-yl]-1-(pyrrolidin-1-yl)ethanone (2cam). Light yellow solid; Yield: 99\%. ${ }^{1} \mathrm{H}-\mathrm{NMR}\left(\mathrm{CDCl}_{3}\right) \delta 1.92(\mathrm{tt}, J=6.8,6.9 \mathrm{~Hz}, 2 \mathrm{H}), 2.05(\mathrm{tt}, J=6.8,6.9 \mathrm{~Hz}, 2 \mathrm{H}), 3.53(\mathrm{t}$, $J=7.0 \mathrm{~Hz}, 2 \mathrm{H}), 3.58(\mathrm{t}, J=6.9 \mathrm{~Hz}, 2 \mathrm{H}), 5.19(\mathrm{~s}, 2 \mathrm{H}), 7.04-7.07(\mathrm{~m}, 4 \mathrm{H}), 7.12(\mathrm{~m}, 1 \mathrm{H}), 7.34-7.37(\mathrm{~m}, 2 \mathrm{H})$, $7.81(\mathrm{~d}, J=8.8 \mathrm{~Hz}, 2 \mathrm{H}), 8.00(\mathrm{~s}, 1 \mathrm{H}) ;{ }^{13} \mathrm{C}-\mathrm{NMR}\left(\mathrm{DMSO}-d_{6}\right) \delta 23.7,25.6,45.1,45.8,51.4,118.8,118.9$, 122.6, 123.6, 126.2, 126.8, 130.1, 145.6, 156.3, 156.4, 163.7; LC-MS (ESI) $m / z 349\left([\mathrm{M}+1]^{+}\right)$.

2-[4-(2,4-Difluorophenyl)-1H-1,2,3-triazol-1-yl]-1-(pyrrolidin-1-yl)ethanone (2can). Light yellow solid; Yield: 99\%. ${ }^{1} \mathrm{H}-\mathrm{NMR}\left(\mathrm{CDCl}_{3}\right)$ 8. $1.92(\mathrm{tt}, J=6.7,6.9 \mathrm{~Hz}, 2 \mathrm{H}), 2.05(\mathrm{tt}, J=6.8,6.9 \mathrm{~Hz}, 2 \mathrm{H}), 3.53(\mathrm{t}$, $J=7.0 \mathrm{~Hz}, 2 \mathrm{H}), 3.58(\mathrm{t}, J=6.9 \mathrm{~Hz}, 2 \mathrm{H}), 5.20(\mathrm{~s}, 2 \mathrm{H}), 6.90(\mathrm{ddd}, J=2.4,8.7,11.0 \mathrm{~Hz}, 1 \mathrm{H}), 6.99(\mathrm{~m}, 1 \mathrm{H})$, $8.14(\mathrm{~d}, J=3.7 \mathrm{~Hz}, 1 \mathrm{H}), 8.27(\mathrm{dt}, J=6.5,8.6 \mathrm{~Hz}, 1 \mathrm{H}) ;{ }^{13} \mathrm{C}-\mathrm{NMR}$ (DMSO- $\left.d_{6}\right) \delta 23.7,25.6,45.1,45.8$, 51.4, 104.6 (t, $\left.J_{\mathrm{CF}}=26.0 \mathrm{~Hz}\right), 112.3\left(\mathrm{dd}, J_{\mathrm{CF}}=20.2,4.8 \mathrm{~Hz}\right), 115.3\left(\mathrm{dd}, J_{\mathrm{CF}}=13.4,3.8 \mathrm{~Hz}\right), 125.0(\mathrm{~d}$, $\left.J_{\mathrm{CF}}=11.1 \mathrm{~Hz}\right), 128.5\left(\mathrm{dd}, J_{\mathrm{CF}}=9.8,5.3 \mathrm{~Hz}\right), 138.7,158.5\left(\mathrm{dd}, J_{\mathrm{CF}}=250.0,12.6 \mathrm{~Hz}\right), 161.7(\mathrm{dd}$, $\left.J_{\mathrm{CF}}=247.4,12.7 \mathrm{~Hz}\right), 163.6$; LC-MS (ESI) $\mathrm{m} / z 293\left([\mathrm{M}+1]^{+}\right)$.

2-(4-Benzyl-1H-1,2,3-triazol-1-yl)-1-(pyrrolidin-1-yl)ethanone (2cao). White solid; Yield: 94\%. ${ }^{1} \mathrm{H}-\mathrm{NMR}$ $\left(\mathrm{CDCl}_{3}\right) \delta 1.86(\mathrm{tt}, J=6.8,7.0 \mathrm{~Hz}, 2 \mathrm{H}), 1.99(\mathrm{tt}, J=6.8,7.0 \mathrm{~Hz}, 2 \mathrm{H}), 3.46(\mathrm{t}, J=7.0 \mathrm{~Hz}, 2 \mathrm{H}), 3.49(\mathrm{t}$, $J=6.9 \mathrm{~Hz}, 2 \mathrm{H}), 4.08(\mathrm{~s}, 2 \mathrm{H}), 5.05(\mathrm{~s}, 2 \mathrm{H}), 7.19(\mathrm{~m}, 1 \mathrm{H}), 7.24-7.29(\mathrm{~m}, 4 \mathrm{H}), 7.42(\mathrm{~s}, 1 \mathrm{H}) ;{ }^{13} \mathrm{C}-\mathrm{NMR}$ (DMSO- $d_{6}$ ) $\delta 23.7,25.6,31.2,45.1,45.7,51.1,124.0,126.1,128.4,128.5,139.7,145.6,163.8$; LC-MS (ESI) $m / z 271\left([\mathrm{M}+1]^{+}\right)$.

1-(Azepan-1-yl)-2-(4-phenyl-1H-1,2,3-triazol-1-yl)ethanone (2daa). Light yellow solid; Yield: 95\%. ${ }^{1} \mathrm{H}-\mathrm{NMR}\left(\mathrm{CDCl}_{3}\right) \delta 1.58-1.63(\mathrm{~m}, 4 \mathrm{H}), 1.72-1.77(\mathrm{~m}, 2 \mathrm{H}), 1.78-1.83(\mathrm{~m}, 2 \mathrm{H}), 3.57(\mathrm{t}, J=6.0 \mathrm{~Hz}, 2 \mathrm{H})$, $3.58(\mathrm{t}, J=6.0 \mathrm{~Hz}, 2 \mathrm{H}), 5.27(\mathrm{~s}, 2 \mathrm{H}), 7.33(\mathrm{~m}, 1 \mathrm{H}), 7.41-7.44(\mathrm{~m}, 2 \mathrm{H}), 7.84-7.86(\mathrm{~m}, 2 \mathrm{H}), 8.04(\mathrm{~s}, 1 \mathrm{H})$; ${ }^{13}$ C-NMR (DMSO- $d_{6}$ ) $\delta 26.2,26.7,27.1,28.2,45.5,46.4,50.7,123.2,125.1,277,128.9,130.9,146.0,165.0$; LC-MS (ESI) $m / z 285\left([\mathrm{M}+1]^{+}\right)$.

1-(Azepan-1-yl)-2-[4-(4-methoxyphenyl)-1H-1,2,3-triazol-1-yl]ethanone (2dab). White solid; Yield: 86\%. ${ }^{1} \mathrm{H}-\mathrm{NMR}\left(\mathrm{CDCl}_{3}\right) \delta 1.59-1.63(\mathrm{~m}, 4 \mathrm{H}), 1.72-1.76(\mathrm{~m}, 2 \mathrm{H}), 1.77-1.82(\mathrm{~m}, 2 \mathrm{H}), 3.53-3.59(\mathrm{~m}, 4 \mathrm{H}), 3.84(\mathrm{~s}$, $3 \mathrm{H}), 5.25(\mathrm{~s}, 2 \mathrm{H}), 6.95(\mathrm{~d}, J=8.9 \mathrm{~Hz}, 2 \mathrm{H}), 7.77(\mathrm{~d}, J=8.8 \mathrm{~Hz}, 2 \mathrm{H}), 7.95(\mathrm{~s}, 1 \mathrm{H}) ;{ }^{13} \mathrm{C}-\mathrm{NMR}\left(\mathrm{DMSO}-d_{6}\right) \delta$ 26.2, 26.7, 27.1, 28.2, 45.5, 46.4, 50.7, 55.1, 114.3, 122.2, 123.5, 126.4, 145.9, 158.9, 165.0; LC-MS (ESI) $\mathrm{m} / \mathrm{z}$ $315\left([\mathrm{M}+1]^{+}\right)$.

4-\{1-[2-(Azepan-1-yl)-2-oxoethyl]-1H-1,2,3-triazol-4-yl\}benzonitrile (2dac). Yellow solid; Yield: 97\%. ${ }^{1} \mathrm{H}-\mathrm{NMR}\left(\mathrm{CDCl}_{3}\right) \delta 1.57-1.66(\mathrm{~m}, 4 \mathrm{H}), 1.75(\mathrm{tt}, J=5.9,6.0 \mathrm{~Hz}, 2 \mathrm{H}), 1.84(\mathrm{tt}, J=5.9,6.0 \mathrm{~Hz}, 2 \mathrm{H})$, $3.56-3.60(\mathrm{~m}, 4 \mathrm{H}), 5.29(\mathrm{~s}, 2 \mathrm{H}), 7.71(\mathrm{~d}, J=6.7 \mathrm{~Hz}, 2 \mathrm{H}), 7.96(\mathrm{~d}, J=6.7 \mathrm{~Hz}, 2 \mathrm{H}), 8.15(\mathrm{~s}, 1 \mathrm{H}) ;{ }^{13} \mathrm{C}-\mathrm{NMR}$ $\left(\right.$ DMSO- $\left.d_{6}\right) \delta 26.2,26.4,27.0,28.1,45.5,46.4,50.9,110.0,118.8,124.9,125.6,133.0,133.3,144.5,164.8$; LC-MS (ESI) $m / z 310\left([\mathrm{M}+1]^{+}\right)$.

1-(Azepan-1-yl)-2-[4-(thiophen-3-yl)-1H-1,2,3-triazol-1-yl]ethanone (2dad). Light brown solid; Yield: 98\%. ${ }^{1} \mathrm{H}-\mathrm{NMR}\left(\mathrm{CDCl}_{3}\right) \delta 1.58-1.61(\mathrm{~m}, 4 \mathrm{H}), 1.75(\mathrm{tt}, J=5.8,5.8 \mathrm{~Hz}, 2 \mathrm{H}), 1.80(\mathrm{tt}, J=5.8,5.9 \mathrm{~Hz}$, $2 \mathrm{H}), 3.57(\mathrm{t}, J=6.0 \mathrm{~Hz}, 2 \mathrm{H}), 3.58(\mathrm{t}, J=5.9 \mathrm{~Hz}, 2 \mathrm{H}), 5.26(\mathrm{~s}, 2 \mathrm{H}), 7.37(\mathrm{dd}, J=3.0,5.0 \mathrm{~Hz}, 1 \mathrm{H}), 7.47(\mathrm{dd}$, $J=1.3,5.0 \mathrm{~Hz}, 1 \mathrm{H}), 7.69(\mathrm{dd}, J=1.2,3.0 \mathrm{~Hz}, 1 \mathrm{H}), 7.94(\mathrm{~s}, 1 \mathrm{H}) ;{ }^{13} \mathrm{C}-\mathrm{NMR}\left(\mathrm{DMSO}-d_{6}\right) \delta 26.2,26.7,27.1$, 28.2, 45.5, 46.4, 50.7, 120.6, 122.9, 125.8, 127.0, 132.2, 142.6, 165.0; LC-MS (ESI) $m / z 291\left([\mathrm{M}+1]^{+}\right)$.

1-(Azepan-1-yl)-2-[4-(pyridin-2-yl)-1H-1,2,3-triazol-1-yl]ethanone (2dae). Light brown solid; Yield: 86\%. ${ }^{1} \mathrm{H}-\mathrm{NMR}\left(\mathrm{CDCl}_{3}\right) \delta 1.59-1.64(\mathrm{~m}, 4 \mathrm{H}), 1.74(\mathrm{tt}, J=5.9,6.0 \mathrm{~Hz}, 2 \mathrm{H}), 1.80(\mathrm{tt}, J=5.8,6.0 \mathrm{~Hz}, 2 \mathrm{H}), 3.55(\mathrm{t}$, 
$J=6.1 \mathrm{~Hz}, 2 \mathrm{H}), 3.57(\mathrm{t}, J=6.0 \mathrm{~Hz}, 2 \mathrm{H}), 5.28(\mathrm{~s}, 2 \mathrm{H}), 7.22(\mathrm{ddd}, J=1.1,4.9,7.5 \mathrm{~Hz}, 1 \mathrm{H}), 7.76(\mathrm{dt}, J=1.8$, $7.7 \mathrm{~Hz}, 1 \mathrm{H}), 8.15(\mathrm{~d}, J=7.9 \mathrm{~Hz}, 1 \mathrm{H}), 8.34(\mathrm{~s}, 1 \mathrm{H}), 8.59(\mathrm{~d}, J=4.3 \mathrm{~Hz}, 1 \mathrm{H}) ;{ }^{13} \mathrm{C}-\mathrm{NMR}$ (DMSO- $\left.d_{6}\right) \delta 26.2$, $26.7,27.0,28.2,45.5,46.4,50.8,119.3,122.9,125.1,137.2,146.9,149.6,150.1,164.9$; LC-MS (ESI) $\mathrm{m} / z 286$ $\left([\mathrm{M}+1]^{+}\right)$.

1-(Azepan-1-yl)-2-[4-(3-methoxyphenyl)-1H-1,2,3-triazol-1-yl]ethanone (2dag). Light yellow solid; Yield: 92\%. ${ }^{1} \mathrm{H}-\mathrm{NMR}\left(\mathrm{CDCl}_{3}\right) \delta 1.58-1.61(\mathrm{~m}, 4 \mathrm{H}), 1.75(\mathrm{~m}, 2 \mathrm{H}), 1.80(\mathrm{~m}, 2 \mathrm{H}), 3.56-3.59(\mathrm{~m}, 4 \mathrm{H}), 3.87(\mathrm{~s}, 3 \mathrm{H})$, $5.27(\mathrm{~s}, 2 \mathrm{H}), 6.88(\mathrm{ddd}, J=1.0,2.6,8.2 \mathrm{~Hz}, 1 \mathrm{H}), 7.32(\mathrm{t}, J=7.9 \mathrm{~Hz}, 1 \mathrm{H}), 7.39(\mathrm{td}, J=1.2,7.6 \mathrm{~Hz}, 1 \mathrm{H}), 7.46$ $(\mathrm{dd}, J=1.5,2.5 \mathrm{~Hz}, 1 \mathrm{H}), 8.03(\mathrm{~s}, 1 \mathrm{H}) ;{ }^{13} \mathrm{C}-\mathrm{NMR}\left(\mathrm{DMSO}-d_{6}\right) \delta 26.2,26.7,27.1,28.2,45.5,46.4,50.8,55.1$, 110.2, 113.5, 117.4, 123.4, 130.0, 132.2, 145.9, 159.7, 165.0; LC-MS (ESI) $\mathrm{m} / \mathrm{z} 315\left([\mathrm{M}+1]^{+}\right)$.

1-(Azepan-1-yl)-2-[4-(2-methoxyphenyl)-1H-1,2,3-triazol-1-yl]ethanone (2dah). White solid; Yield: 88\%. ${ }^{1} \mathrm{H}-\mathrm{NMR}\left(\mathrm{CDCl}_{3}\right) \delta 1.55-1.61(\mathrm{~m}, 4 \mathrm{H}), 1.72-1.78(\mathrm{~m}, 4 \mathrm{H}), 3.56-3.59(\mathrm{~m}, 4 \mathrm{H}), 3.93(\mathrm{~s}, 3 \mathrm{H}), 5.27(\mathrm{~s}, 2 \mathrm{H})$, $6.97(\mathrm{dd}, J=0.7,8.3 \mathrm{~Hz}, 1 \mathrm{H}), 7.07(\mathrm{dt}, J=1.0,7.6 \mathrm{~Hz}, 1 \mathrm{H}), 7.31(\mathrm{ddd}, J=1.8,7.4,8.3 \mathrm{~Hz}, 1 \mathrm{H}), 8.27(\mathrm{~s}$, $1 \mathrm{H}), 8.34(\mathrm{dd}, J=1.7,7.7 \mathrm{~Hz}, 1 \mathrm{H}) ;{ }^{13} \mathrm{C}-\mathrm{NMR}\left(\mathrm{DMSO}-d_{6}\right) \delta 26.2,26.7,27.1,28.1,45.4,46.4,50.5,55.5$, $111.5,119.2,120.6,125.7,126.4,128.7,141.4,155.3,165.2$; LC-MS (ESI) $\mathrm{m} / z 315\left([\mathrm{M}+1]^{+}\right)$.

1-(Azepan-1-yl)-2-(4-p-tolyl-1H-1,2,3-triazol-1-yl)ethanone (2dai). White solid; Yield: 97\%. ${ }^{1} \mathrm{H}-\mathrm{NMR}$ $\left(\mathrm{CDCl}_{3}\right) \delta 1.55-1.62(\mathrm{~m}, 4 \mathrm{H}), 1.74(\mathrm{~m}, 2 \mathrm{H}), 1.79(\mathrm{~m}, 2 \mathrm{H}), 2.38(\mathrm{~s}, 3 \mathrm{H}), 3.55-3.59(\mathrm{~m}, 4 \mathrm{H}), 5.26(\mathrm{~s}, 2 \mathrm{H})$, $7.23(\mathrm{~d}, J=7.9 \mathrm{~Hz}, 2 \mathrm{H}), 7.73(\mathrm{~d}, J=8.1 \mathrm{~Hz}, 2 \mathrm{H}), 7.99(\mathrm{~s}, 1 \mathrm{H}) ;{ }^{13} \mathrm{C}-\mathrm{NMR}\left(\mathrm{DMSO}-d_{6}\right) \delta 20.8,26.2,26.7$, 27.1, 28.2, 45.5, 46.5, 50.7, 122.7, 125.0, 128.1, 129.4, 137.0, 146.1, 165.0; LC-MS (ESI) $m / z 299\left([\mathrm{M}+1]^{+}\right)$.

1-(Azepan-1-yl)-2-(4-m-tolyl-1H-1,2,3-triazol-1-yl)ethanone (2daj). White solid; Yield: 91\%. ${ }^{1} \mathrm{H}-\mathrm{NMR}$ $\left(\mathrm{CDCl}_{3}\right) \delta 1.56-1.62(\mathrm{~m}, 4 \mathrm{H}), 1.74(\mathrm{~m}, 2 \mathrm{H}), 1.79(\mathrm{~m}, 2 \mathrm{H}), 2.40(\mathrm{~s}, 3 \mathrm{H}), 3.55-3.59(\mathrm{~m}, 4 \mathrm{H}), 5.26(\mathrm{~s}, 2 \mathrm{H})$, $7.15(\mathrm{dd}, J=0.6,7.6 \mathrm{~Hz}, 1 \mathrm{H}), 7.30(\mathrm{t}, J=7.7 \mathrm{~Hz}, 1 \mathrm{H}), 7.62(\mathrm{~d}, J=7.8 \mathrm{~Hz}, 1 \mathrm{H}), 7.71(\mathrm{~s}, 1 \mathrm{H}), 8.02(\mathrm{~s}, 1 \mathrm{H})$; ${ }^{13}$ C-NMR (DMSO- $d_{6}$ ) $\delta 21.1,26.2,26.7,27.0,28.2,45.5,46.5,50.7,122.2,123.1,125.6,128.4,128.8,130.8$, 138.0, 146.1, 165.0; LC-MS (ESI) $\mathrm{m} / z 299\left([\mathrm{M}+1]^{+}\right)$.

1-(Azepan-1-yl)-2-(4-o-tolyl-1H-1,2,3-triazol-1-yl)ethanone (2dak). Light yellow solid; Yield: 86\%. ${ }^{1} \mathrm{H}-\mathrm{NMR}\left(\mathrm{CDCl}_{3}\right) \delta 1.58-1.63(\mathrm{~m}, 4 \mathrm{H}), 1.75(\mathrm{~m}, 2 \mathrm{H}), 1.80(\mathrm{~m}, 2 \mathrm{H}), 2.49(\mathrm{~s}, 3 \mathrm{H}), 3.57-3.60(\mathrm{~m}, 4 \mathrm{H})$, $5.29(\mathrm{~s}, 2 \mathrm{H}), 7.26-7.28(\mathrm{~m}, 3 \mathrm{H}), 7.78(\mathrm{~m}, 1 \mathrm{H}), 7.92(\mathrm{~s}, 1 \mathrm{H}) ;{ }^{13} \mathrm{C}-\mathrm{NMR}$ (DMSO- $\left.d_{6}\right) \delta 21.1,26.2,26.7,27.1$, $28.2,45.5,46.4,50.7,125.1,126.0,127.7,128.1,130.1,130.9,134.8,145.1,165.0$; LC-MS (ESI) $\mathrm{m} / z 299$ $\left([\mathrm{M}+1]^{+}\right)$.

1-(Azepan-1-yl)-2-\{4-[4-(dimethylamino)phenyl]-1H-1,2,3-triazol-1-yl\}ethanone (2dal). Light brown solid; Yield: $89 \% .{ }^{1} \mathrm{H}-\mathrm{NMR}\left(\mathrm{CDCl}_{3}\right) \delta 1.57-1.58(\mathrm{~m}, 4 \mathrm{H}), 1.73-1.77(\mathrm{~m}, 4 \mathrm{H}), 2.99(\mathrm{~s}, 6 \mathrm{H}), 3.56(\mathrm{t}, J=6.0 \mathrm{~Hz}$, $2 \mathrm{H}), 3.57(\mathrm{t}, J=6.0 \mathrm{~Hz}, 2 \mathrm{H}), 5.23(\mathrm{~s}, 2 \mathrm{H}), 6.77(\mathrm{~d}, J=8.9 \mathrm{~Hz}, 2 \mathrm{H}), 7.71(\mathrm{~d}, J=8.9 \mathrm{~Hz}, 2 \mathrm{H}), 7.89(\mathrm{~s}, 1 \mathrm{H})$; ${ }^{13}$ C-NMR (DMSO- $d_{6}$ ) $\delta 26.2,26.7,27.1,28.2,40.0,45.5,46.5,50.6,112.4,118.8,121.3,126.0,146.6,150.0$, 165.1; LC-MS (ESI) $m / z 328\left([\mathrm{M}+1]^{+}\right)$.

1-(Azepan-1-yl)-2-[4-(4-phenoxyphenyl)-1H-1,2,3-triazol-1-yl]ethanone (2dam). Light yellow solid; Yield: 99\%. ${ }^{1} \mathrm{H}-\mathrm{NMR}\left(\mathrm{CDCl}_{3}\right) \delta 1.59-1.66(\mathrm{~m}, 4 \mathrm{H}), 1.73-1.77(\mathrm{~m}, 2 \mathrm{H}), 1.7-1.83(\mathrm{~m}, 2 \mathrm{H}), 3.57(\mathrm{t}, J=6.0 \mathrm{~Hz}$, $2 \mathrm{H}), 3.58(\mathrm{t}, J=6.0 \mathrm{~Hz}, 2 \mathrm{H}), 5.27(\mathrm{~s}, 2 \mathrm{H}), 7.04-7.06(\mathrm{~m}, 4 \mathrm{H}), 7.12(\mathrm{~m}, 1 \mathrm{H}), 7.34-7.37(\mathrm{~m}, 2 \mathrm{H}), 7.81(\mathrm{~d}$, $J=8.9 \mathrm{~Hz}, 2 \mathrm{H}), 7.99(\mathrm{~s}, 1 \mathrm{H}) ;{ }^{13} \mathrm{C}-\mathrm{NMR}\left(\mathrm{DMSO}-d_{6}\right) \delta 26.2,26.7,27.1,28.2,45.5,46.5,50.7,118.8,118.9$, 122.8, 123.6, 126.2, 126.8, 130.1, 145.6, 456.3, 156.4, 165.0; LC-MS (ESI) $m / z 377\left([\mathrm{M}+1]^{+}\right)$.

1-(Azepan-1-yl)-2-[4-(2,4-difluorophenyl)-1H-1,2,3-triazol-1-yl]ethanone (2dan). Yellow solid; Yield: 98\%. ${ }^{1} \mathrm{H}-\mathrm{NMR}\left(\mathrm{CDCl}_{3}\right) \delta 1.57-1.65(\mathrm{~m}, 4 \mathrm{H}), 1.75(\mathrm{tt}, J=5.8,5.9 \mathrm{~Hz}, 2 \mathrm{H}), 1.82(\mathrm{tt}, J=5.8,5.9 \mathrm{~Hz}, 2 \mathrm{H}), 3.56(\mathrm{t}$, $J=6.1 \mathrm{~Hz}, 2 \mathrm{H}), 3.58(\mathrm{t}, J=6.1 \mathrm{~Hz}, 2 \mathrm{H}), 5.28(\mathrm{~s}, 2 \mathrm{H}), 6.90(\mathrm{ddd}, J=2.4,8.7,11.0 \mathrm{~Hz}, 1 \mathrm{H}), 6.99$ (dddd, $J=1.0,2.5,8.0,10.4 \mathrm{~Hz}, 1 \mathrm{H}), 8.13(\mathrm{~d}, J=3.7 \mathrm{~Hz}, 1 \mathrm{H}), 8.27(\mathrm{dt}, J=6.5,8.6 \mathrm{~Hz}, 1 \mathrm{H}) ;{ }^{13} \mathrm{C}-\mathrm{NMR}\left(\mathrm{DMSO}-d_{6}\right)$ $\delta 26.2,26.7,27.1,28.1,45.5,46.4,50.8,104.6\left(\mathrm{t}, J_{\mathrm{CF}}=26.0 \mathrm{~Hz}\right), 112.3\left(\mathrm{dd}, J_{\mathrm{CF}}=20.2,4.8 \mathrm{~Hz}\right), 115.3(\mathrm{dd}$, $\left.J_{\mathrm{CF}}=13.2,3.7 \mathrm{~Hz}\right), 125.3\left(\mathrm{~d}, J_{\mathrm{CF}}=10.9 \mathrm{~Hz}\right), 128.5\left(\mathrm{dd}, J_{\mathrm{CF}}=9.7,5.4 \mathrm{~Hz}\right), 138.7\left(\mathrm{~d}, J_{\mathrm{CF}}=2.4 \mathrm{~Hz}\right), 158.5$ $\left(\mathrm{dd}, J_{\mathrm{CF}}=249.9,12.5 \mathrm{~Hz}\right), 161.7\left(\mathrm{dd}, J_{\mathrm{CF}}=247.4,12.7 \mathrm{~Hz}\right), 165.0$; LC-MS (ESI) $\mathrm{m} / z 321\left([\mathrm{M}+1]^{+}\right)$. 
1-(Azepan-1-yl)-2-(4-benzyl-1H-1,2,3-triazol-1-yl)ethanone (2dao). White solid; Yield: $94 \% .{ }^{1} \mathrm{H}-\mathrm{NMR}$ $\left(\mathrm{CDCl}_{3}\right) \delta 1.55-1.57(\mathrm{~m}, 4 \mathrm{H}), 1.68-1.75(\mathrm{~m}, 4 \mathrm{H}), 3.50(\mathrm{t}, J=6.1 \mathrm{~Hz}, 2 \mathrm{H}), 3.52(\mathrm{t}, J=6.1 \mathrm{~Hz}, 2 \mathrm{H}), 4.10(\mathrm{~s}$, $2 \mathrm{H}), 5.15(\mathrm{~s}, 2 \mathrm{H}), 7.21(\mathrm{~m}, 1 \mathrm{H}), 7.26-7.30(\mathrm{~m}, 4 \mathrm{H}), 7.41(\mathrm{~s}, 1 \mathrm{H}) ;{ }^{13} \mathrm{C}-\mathrm{NMR}$ (DMSO- $\left.d_{6}\right) \delta 26.2,26.7,27.1$, 28.1, 31.3, 45.5, 46.5, 50.5, 124.3, 126.1, 128.4, 128.6, 139.7, 145.7, 165.1; LC-MS (ESI) $\mathrm{m} / z 299\left([\mathrm{M}+1]^{+}\right)$.

N-Methyl-N-phenyl-2-(4-phenyl-1H-1,2,3-triazol-1-yl)acetamide (2eaa). White solid; Yield: 99\%. ${ }^{1} \mathrm{H}-\mathrm{NMR}\left(\mathrm{CDCl}_{3}\right) \delta 3.35(\mathrm{~s}, 3 \mathrm{H}), 4.97(\mathrm{~s}, 2 \mathrm{H}), 7.31-7.34(\mathrm{~m}, 3 \mathrm{H}), 7.40-7.43(\mathrm{~m}, 2 \mathrm{H}), 7.45(\mathrm{~m}, 1 \mathrm{H})$, 7.51-7.54 (m, 2H), 7.82-7.84 (m, 2H), $7.99(\mathrm{~s}, 1 \mathrm{H}) ;{ }^{13} \mathrm{C}-\mathrm{NMR}\left(\mathrm{DMSO}-d_{6}\right) \delta 37.3,51.1,123.0,125.1,127.5$, $127.8,128.4,129.0,130.1,130.8,141.9,146.0,164.9$; LC-MS (ESI) $\mathrm{m} / z 293\left([\mathrm{M}+1]^{+}\right)$.

2-[4-(4-Methoxyphenyl)-1H-1,2,3-triazol-1-yl]-N-methyl-N-phenylacetamide (2eab). Yellow solid; Yield: 99\%. ${ }^{1} \mathrm{H}-\mathrm{NMR}\left(\mathrm{CDCl}_{3}\right) \delta 3.35(\mathrm{~s}, 3 \mathrm{H}), 3.84(\mathrm{~s}, 3 \mathrm{H}), 4.95(\mathrm{~s}, 2 \mathrm{H}), 6.95(\mathrm{~d}, J=8.9 \mathrm{~Hz}, 2 \mathrm{H}), 7.30-7.32$ $(\mathrm{m}, 2 \mathrm{H}), 7.45(\mathrm{~m}, 1 \mathrm{H}), 7.50-7.53(\mathrm{~m}, 2 \mathrm{H}), 7.75(\mathrm{~d}, J=8.9 \mathrm{~Hz}, 2 \mathrm{H}), 7.89(\mathrm{~s}, 1 \mathrm{H}) ;{ }^{13} \mathrm{C}-\mathrm{NMR}\left(\right.$ DMSO- $\left.d_{6}\right) \delta$ 37.3, 51.0, 55.2, 114.3, 122.0, 123.4, 126.5, 127.5, 128.4, 130.1, 141.9, 146.0, 159.0, 164.9; LC-MS (ESI) $\mathrm{m} / \mathrm{z}$ $323\left([\mathrm{M}+1]^{+}\right)$.

2-[4-(4-Cyanophenyl)-1H-1,2,3-triazol-1-yl]-N-methyl-N-phenylacetamide (2eac). White solid; Yield: 99\%. ${ }^{1} \mathrm{H}-\mathrm{NMR}\left(\mathrm{CDCl}_{3}\right) \delta 3.35(\mathrm{~s}, 3 \mathrm{H}), 4.98(\mathrm{~s}, 2 \mathrm{H}), 7.30-7.34(\mathrm{~m}, 2 \mathrm{H}), 7.47(\mathrm{~m}, 1 \mathrm{H}), 7.56-7.51(\mathrm{~m}, 2 \mathrm{H}), 7.70$ $(\mathrm{d}, J=8.6 \mathrm{~Hz}, 2 \mathrm{H}), 7.94(\mathrm{~d}, J=8.6 \mathrm{~Hz}, 2 \mathrm{H}), 8.10(\mathrm{~s}, 1 \mathrm{H}) ;{ }^{13} \mathrm{C}-\mathrm{NMR}\left(\mathrm{CDCl}_{3}\right) \delta 37.9,51.4,111.4,118.9$, $122.9,126.2,127.3,129.2,130.6,132.7,135.1,141.7,146.0,164.7$; LC-MS (ESI) $m / z 318\left([\mathrm{M}+1]^{+}\right)$.

N-Methyl-N-phenyl-2-[4-(thiophen-3-yl)-1H-1,2,3-triazol-1-yl]acetamide (2ead). White solid; Yield: 89\%. ${ }^{1} \mathrm{H}-\mathrm{NMR}\left(\mathrm{CDCl}_{3}\right) \delta 3.34(\mathrm{~s}, 3 \mathrm{H}), 4.97(\mathrm{~s}, 2 \mathrm{H}), 7.21(\mathrm{ddd}, J=1.1,4.9,7.5 \mathrm{~Hz}, 1 \mathrm{H}), 7.32(\mathrm{~d}, J=7.3 \mathrm{~Hz}$, $2 \mathrm{H}), 7.45(\mathrm{t}, J=7.4 \mathrm{~Hz}, 1 \mathrm{H}), 7.51(\mathrm{t}, J=7.6 \mathrm{~Hz}, 2 \mathrm{H}), 7.75(\mathrm{dt}, J=1.8,7.7 \mathrm{~Hz}, 1 \mathrm{H}), 8.13(\mathrm{~d}, J=7.9 \mathrm{~Hz}$, $1 \mathrm{H}), 8.29(\mathrm{~s}, 1 \mathrm{H}), 8.58\left(\mathrm{ddd}, J=4.9,1.6,0.9 \mathrm{~Hz}, 1 \mathrm{H} ;{ }^{13} \mathrm{C}-\mathrm{NMR}\left(\mathrm{CDCl}_{3}\right) \delta 37.9,51.3,121.1,121.4,126.0\right.$, $126.3,127.4,129.1,130.6,132.0,141.9,144.1,164.9$; LC-MS (ESI) $\mathrm{m} / z 294\left([\mathrm{M}+1]^{+}\right)$.

N-Methyl-N-phenyl-2-[4-(pyridin-2-yl)-1H-1,2,3-triazol-1-yl]acetamide (2eae). White solid; Yield: 99\%. ${ }^{1} \mathrm{H}-\mathrm{NMR}\left(\mathrm{CDCl}_{3}\right) \delta 3.34(\mathrm{~s}, 3 \mathrm{H}), 4.94(\mathrm{~s}, 2 \mathrm{H}), 7.30(\mathrm{~d}, J=7.4 \mathrm{~Hz}, 2 \mathrm{H}), 7.36(\mathrm{ddd}, J=5.0,3.0,0.6 \mathrm{~Hz}, 1 \mathrm{H})$, 7.42-7.48 (m, 2H), 7.48-7.54 (m, 2H), $7.67(\mathrm{~m}, 1 \mathrm{H}), 7.88(\mathrm{~s}, 1 \mathrm{H}) ;{ }^{13} \mathrm{C}-\mathrm{NMR}\left(\mathrm{CDCl}_{3}\right) \delta 37.9,51.4,120.3$, 122.8, 124.0, 127.4, 129.1, 130.6, 136.8, 142.0, 148.6, 149.5, 150.4, 164.7; LC-MS (ESI) $m / z 299\left([\mathrm{M}+1]^{+}\right)$.

2-[4-(3-Methoxyphenyl)-1H-1,2,3-triazol-1-yl)-N-methyl-N-phenylacetamide (2eag). White solid; Yield: 93\%. ${ }^{1} \mathrm{H}-\mathrm{NMR}\left(\mathrm{CDCl}_{3}\right) \delta 3.35(\mathrm{~s}, 3 \mathrm{H}), 3.86(\mathrm{~s}, 3 \mathrm{H}), 4.96(\mathrm{~s}, 2 \mathrm{H}), 6.88(\mathrm{ddd}, J=1.1,2.6,8.1 \mathrm{~Hz}, 1 \mathrm{H})$, $7.30-7.33(\mathrm{~m}, 3 \mathrm{H}), 7.37(\mathrm{td}, J=1.3,7.6 \mathrm{~Hz}, 1 \mathrm{H}), 7.44(\mathrm{dd}, J=1.5,2.6 \mathrm{~Hz}, 1 \mathrm{H}), 7.45(\mathrm{~m}, 1 \mathrm{H}), 7.50-7.53(\mathrm{~m}$, 2H), $7.98(\mathrm{~s}, 1 \mathrm{H}) ;{ }^{13} \mathrm{C}-\mathrm{NMR}$ (DMSO- $\left.d_{6}\right) \delta 37.3,51.1,55.1,110.3,113.6,117.5,123.2,127.5,128.4,130.1$, 132.1 (2C), 141.9, 146.0, 159.7, 164.9; LC-MS (ESI) $m / z 315\left([\mathrm{M}+1]^{+}\right)$.

2-[4-(2-Methoxyphenyl)-1H-1,2,3-triazol-1-yl)-N-methyl-N-phenylacetamide (2eah). Light yellow solid; Yield: 95\%. ${ }^{1} \mathrm{H}-\mathrm{NMR}\left(\mathrm{CDCl}_{3}\right) \delta 3.35(\mathrm{~s}, 3 \mathrm{H}), 3.93(\mathrm{~s}, 3 \mathrm{H}), 4.97(\mathrm{~s}, 2 \mathrm{H}), 6.97(\mathrm{~d}, J=8.3 \mathrm{~Hz}, 1 \mathrm{H}), 7.06(\mathrm{dt}$, $J=0.9,7.5 \mathrm{~Hz}, 1 \mathrm{H}), 7.28-7.33(\mathrm{~m}, 3 \mathrm{H}), 7.45(\mathrm{~m}, 1 \mathrm{H}), 7.50-7.53(\mathrm{~m}, 2 \mathrm{H}), 8.22(\mathrm{~s}, 1 \mathrm{H}), 8.33(\mathrm{dd}, J=1.7$, $7.7 \mathrm{~Hz}, 1 \mathrm{H}) ;{ }^{13} \mathrm{C}-\mathrm{NMR}\left(\mathrm{DMSO}-d_{6}\right) \delta 37.5,51.2,55.7,111.8,119.2,120.9,125.9,126.7,127.7,128.7,129.2$, 130.3, 141.7, 142.1, 155.5, 165.3; LC-MS (ESI) $\mathrm{m} / z 315\left([\mathrm{M}+1]^{+}\right)$.

N-Methyl-N-phenyl-2-(4-p-tolyl-1H-1,2,3-triazol-1-yl)acetamide (2eai). White solid; Yield: 99\%. ${ }^{1} \mathrm{H}-\mathrm{NMR}$ $\left(\mathrm{CDCl}_{3}\right) \delta 2.37(\mathrm{~s}, 3 \mathrm{H}), 3.35(\mathrm{~s}, 3 \mathrm{H}), 4.95(\mathrm{~s}, 2 \mathrm{H}), 7.22(\mathrm{~d}, J=7.9 \mathrm{~Hz}, 2 \mathrm{H}), 7.30-7.32(\mathrm{~m}, 2 \mathrm{H}), 7.45(\mathrm{~m}, 1 \mathrm{H})$, $7.50-7.53(\mathrm{~m}, 2 \mathrm{H}), 7.72(\mathrm{~d}, J=8.2 \mathrm{~Hz}, 2 \mathrm{H}), 7.94(\mathrm{~s}, 1 \mathrm{H}) ;{ }^{13} \mathrm{C}-\mathrm{NMR}$ (DMSO- $\left.d_{6}\right) \delta 20.8,37.2,51.0,122.5$, 125.0, 127.4, 128.0, 128.4, 129.4, 130.0, 137.1, 141.9, 146.0, 164.9; LC-MS (ESI) $m / z 299\left([\mathrm{M}+1]^{+}\right)$.

N-Methyl-N-phenyl-2-(4-m-tolyl-1H-1,2,3-triazol-1-yl)acetamide (2eaj). White solid; Yield: 87\%. ${ }^{1} \mathrm{H}-\mathrm{NMR}\left(\mathrm{CDCl}_{3}\right) \delta 2.39(\mathrm{~s}, 3 \mathrm{H}), 3.33(\mathrm{~s}, 3 \mathrm{H}), 4.95(\mathrm{~s}, 2 \mathrm{H}), 7.13(\mathrm{~d}, J=7.6 \mathrm{~Hz}, 1 \mathrm{H}), 7.27-7.31(\mathrm{~m}, 3 \mathrm{H})$, $7.44(\mathrm{~m}, 1 \mathrm{H}), 7.49-7.52(\mathrm{~m}, 2 \mathrm{H}), 7.59(\mathrm{~d}, J=7.8 \mathrm{~Hz}, 1 \mathrm{H}), 7.69(\mathrm{~s}, 1 \mathrm{H}), 7.96(\mathrm{~s}, 1 \mathrm{H}) ;{ }^{13} \mathrm{C}-\mathrm{NMR}$ (DMSO- $\left.d_{6}\right)$ $\delta$ 21.0, 37.3, 51.0, 122.3, 122.9, 125.6, 127.4, 128.4, 128.8, 130.0, 130.7, 138.0, 141.9, 146.1, 164.9; LC-MS (ESI) $m / z 299\left([\mathrm{M}+1]^{+}\right)$. 
N-Methyl-N-phenyl-2-(4-o-tolyl-1H-1,2,3-triazol-1-yl)acetamide (2eak). Colorless oil; Yield: 98. ${ }^{1} \mathrm{H}-\mathrm{NMR}$ $\left(\mathrm{CDCl}_{3}\right) \delta 2.45(\mathrm{~s}, 3 \mathrm{H}), 3.32(\mathrm{~s}, 3 \mathrm{H}), 4.97(\mathrm{~s}, 2 \mathrm{H}), 7.22-7.24(\mathrm{~m}, 3 \mathrm{H}), 7.29-7.31(\mathrm{~m}, 2 \mathrm{H}), 7.42(\mathrm{~m}, 1 \mathrm{H})$, 7.47-7.50 (m, 2H), $7.76(\mathrm{~m}, 1 \mathrm{H}), 7.86(\mathrm{~s}, 1 \mathrm{H}) ;{ }^{13} \mathrm{C}-\mathrm{NMR}$ (DMSO-d $\left.d_{6}\right) \delta 21.1,37.3,51.0,124.9,126.0,127.5$, 127.7, 128.0, 128.4, 130.0, 130.9, 134.7, 141.9, 145.1, 164.9; LC-MS (ESI) $m / z 299\left([\mathrm{M}+1]^{+}\right)$.

1-Morpholino-3-(4-phenyl-1H-1,2,3-triazol-1-yl)propan-1-one (2aca). Colorless oil; Yield: 94\%. ${ }^{1} \mathrm{H}-\mathrm{NMR}$ $\left(\mathrm{CDCl}_{3}\right) \delta 2.96(\mathrm{t}, J=6.2 \mathrm{~Hz}, 2 \mathrm{H}), 3.38(\mathrm{t}, J=4.9 \mathrm{~Hz}, 2 \mathrm{H}), 3.56-3.62(\mathrm{~m}, 6 \mathrm{H}), 4.74(\mathrm{t}, J=6.2 \mathrm{~Hz}, 2 \mathrm{H})$, $7.30(\mathrm{~m}, 1 \mathrm{H}), 7.38-7.41(\mathrm{~m}, 2 \mathrm{H}), 7.78-7.81(\mathrm{~m}, 2 \mathrm{H}), 7.93(\mathrm{~s}, 1 \mathrm{H}) ;{ }^{13} \mathrm{C}-\mathrm{NMR}\left(\mathrm{DMSO}-d_{6}\right) \delta 32.4,41.5,45.2$, $45.8,65.97,65.98,121.8,125.1,127.8,128.9,130.8,146.0,168.0$; LC-MS (ESI) $m / z 287\left([\mathrm{M}+1]^{+}\right)$.

3-[4-(4-Methoxyphenyl)-1H-1,2,3-triazol-1-yl]-1-morpholinopropan-1-one (2acb). Colorless oil; Yield: 90\%. ${ }^{1} \mathrm{H}-\mathrm{NMR}\left(\mathrm{CDCl}_{3}\right) \delta 2.96(\mathrm{t}, J=6.2 \mathrm{~Hz}, 2 \mathrm{H}), 3.38(\mathrm{t}, J=4.9 \mathrm{~Hz}, 2 \mathrm{H}), 3.56-3.62(\mathrm{~m}, 6 \mathrm{H}), 3.81(\mathrm{~s}, 3 \mathrm{H}), 4.72$ $(\mathrm{t}, J=6.2 \mathrm{~Hz}, 2 \mathrm{H}), 6.92(\mathrm{~d}, J=8.8 \mathrm{~Hz}, 2 \mathrm{H}), 7.72(\mathrm{~d}, J=8.8 \mathrm{~Hz}, 2 \mathrm{H}), 7.83(\mathrm{~s}, 1 \mathrm{H}) ;{ }^{13} \mathrm{C}-\mathrm{NMR}\left(\mathrm{DMSO}-d_{6}\right)$ $\delta 32.5,41.5,45.2,45.7,55.1,65.97,65.99,114.3,120.8,123.4,126.4,146.0,158.9,168.1$; LC-MS (ESI) $\mathrm{m} / z$ $317\left([\mathrm{M}+1]^{+}\right)$.

4-[1-(3-Morpholino-3-oxopropyl)-1H-1,2,3-triazol-4-yl]benzonitrile (2acc). White solid; Yield: 90\%. ${ }^{1} \mathrm{H}-\mathrm{NMR}\left(500 \mathrm{MHz}, \mathrm{CDCl}_{3}\right) \delta 3.01(\mathrm{t}, J=5.9 \mathrm{~Hz}, 2 \mathrm{H}), 3.42(\mathrm{t}, J=4.9 \mathrm{~Hz}, 2 \mathrm{H}), 3.59-3.65(\mathrm{~m}, 6 \mathrm{H})$, $4.80(\mathrm{t}, J=5.9 \mathrm{~Hz}, 2 \mathrm{H}), 7.94(\mathrm{~d}, J=8.5 \mathrm{~Hz}, 2 \mathrm{H}), 7.71(\mathrm{~d}, J=8.5 \mathrm{~Hz}, 2 \mathrm{H}), 8.08(\mathrm{~s}, 1 \mathrm{H}) ;{ }^{13} \mathrm{C}$ NMR $(125$ MHz, DMSO-d $\left.d_{6}\right) \delta 32.3,41.5,45.1,46.0,65.95,65.97,110.0,118.8,123.6,125.6,133.0,135.3,144.4,168.0$; LC-MS (ESI) $m / z 312\left([\mathrm{M}+1]^{+}\right)$.

1-Morpholino-3-[4-(thiophen-3-yl)-1H-1,2,3-triazol-1-yl]propan-1-one (2acd). Yellow oil; Yield: 98\%. ${ }^{1} \mathrm{H}-\mathrm{NMR}\left(\mathrm{CDCl}_{3}\right) \delta 2.95(\mathrm{t}, J=6.2 \mathrm{~Hz}, 2 \mathrm{H}), 3.37(\mathrm{t}, J=4.9 \mathrm{~Hz}, 2 \mathrm{H}), 3.55-3.61(\mathrm{~m}, 6 \mathrm{H}), 4.72(\mathrm{t}$, $J=6.1 \mathrm{~Hz}, 2 \mathrm{H}), 7.34(\mathrm{dd}, J=3.0,5.0 \mathrm{~Hz}, 1 \mathrm{H}), 7.42(\mathrm{dd}, J=1.3,5.0 \mathrm{~Hz}, 1 \mathrm{H}), 7.63(\mathrm{dd}, J=1.2,3.0 \mathrm{~Hz}, 1 \mathrm{H})$, $7.83(\mathrm{~s}, 1 \mathrm{H}) ;{ }^{13} \mathrm{C}-\mathrm{NMR}$ (DMSO- $d_{6}$ ) $\delta 32.5,41.5,45.2,45.7,65.96,65.98,120.6,121.6,125.7,127.1,132.1$, 142.6, 168.0; LC-MS (ESI) $\mathrm{m} / \mathrm{z} 293\left([\mathrm{M}+1]^{+}\right)$.

1-Morpholino-3-[4-(pyridin-2-yl)-1H-1,2,3-triazol-1-yl]propan-1-one (2ace). Light yellow oil; Yield: 89\%. ${ }^{1} \mathrm{H}-\mathrm{NMR}\left(\mathrm{CDCl}_{3}\right) \delta 2.99(\mathrm{t}, J=6.5 \mathrm{~Hz}, 2 \mathrm{H}), 3.39(\mathrm{t}, J=4.8 \mathrm{~Hz}, 2 \mathrm{H}), 3.57-3.63(\mathrm{~m}, 6 \mathrm{H}), 4.77(\mathrm{t}, J=6.5 \mathrm{~Hz}$, $2 \mathrm{H}), 7.20(\mathrm{ddd}, J=1.0,4.9,7.5 \mathrm{~Hz}, 1 \mathrm{H}), 7.74(\mathrm{dt}, J=1.7,7.7 \mathrm{~Hz}, 1 \mathrm{H}), 8.11(\mathrm{~d}, J=8.0 \mathrm{~Hz}, 1 \mathrm{H}), 8.25(\mathrm{~s}$, $1 \mathrm{H}), 8.56(\mathrm{~d}, J=4.9 \mathrm{~Hz}, 1 \mathrm{H}) ;{ }^{13} \mathrm{C}-\mathrm{NMR}\left(\mathrm{DMSO}_{6} \mathrm{~d}_{6}\right) \delta 32.4,41.5,45.1,45.8,65.97,65.99,119.3,122.9$, 123.6, 137.2, 147.0, 149.6, 150.0, 168.1; LC-MS (ESI) $m / z 288\left([\mathrm{M}+1]^{+}\right)$.

3-[4-(3-Methoxyphenyl)-1H-1,2,3-triazol-1-yl]-1-morpholinopropan-1-one (2acg). Colorless oil; Yield: 98\%. ${ }^{1} \mathrm{H}-\mathrm{NMR}\left(\mathrm{CDCl}_{3}\right) \delta 2.96(\mathrm{t}, J=6.2 \mathrm{~Hz}, 2 \mathrm{H}), 3.37(\mathrm{t}, J=4.9 \mathrm{~Hz}, 2 \mathrm{H}), 3.56-3.62(\mathrm{~m}, 6 \mathrm{H}), 3.83(\mathrm{~s}, 3 \mathrm{H}), 4.73$ $(\mathrm{t}, J=6.2 \mathrm{~Hz}, 2 \mathrm{H}), 6.85(\mathrm{ddd}, J=1.2,2.6,8.0 \mathrm{~Hz}, 1 \mathrm{H}), 7.29(\mathrm{t}, J=7.8 \mathrm{~Hz}, 1 \mathrm{H}), 7.33(\mathrm{td}, J=1.3,7.6 \mathrm{~Hz}$, $1 \mathrm{H}), 7.40(\mathrm{dd}, J=1.5,2.5 \mathrm{~Hz}, 1 \mathrm{H}), 7.92(\mathrm{~s}, 1 \mathrm{H}) ;{ }^{13} \mathrm{C}-\mathrm{NMR}\left(\mathrm{DMSO}-d_{6}\right) \delta 32.4,41.5,45.2,45.8,55.1,65.98$, 66.00, 110.3, 13.5, 117.5, 122.1, 130.1, 132.2, 146.0, 459.7, 168.0; LC-MS (ESI) $m / z 317\left([\mathrm{M}+1]^{+}\right)$.

3-[4-(2-Methoxyphenyl)-1H-1,2,3-triazol-1-yl]-1-morpholinopropan-1-one (2ach). Light yellow oil; Yield: 99\%. ${ }^{1} \mathrm{H}-\mathrm{NMR}\left(\mathrm{CDCl}_{3}\right) \delta 3.00(\mathrm{t}, J=6.5 \mathrm{~Hz}, 2 \mathrm{H}), 3.37(\mathrm{t}, J=4.9 \mathrm{~Hz}, 2 \mathrm{H}), 3.56-3.61(\mathrm{~m}, 6 \mathrm{H}), 3.91(\mathrm{~s}$, $3 \mathrm{H}), 4.74(\mathrm{t}, J=6.5 \mathrm{~Hz}, 2 \mathrm{H}), 6.95(\mathrm{dd}, J=0.7,8.3 \mathrm{~Hz}, 1 \mathrm{H}), 7.05(\mathrm{dt}, J=1.0,7.5 \mathrm{~Hz}, 1 \mathrm{H}), 7.29$ (ddd, $J=1.7,7.3,8.3 \mathrm{~Hz}, 1 \mathrm{H}), 8.11(\mathrm{~s}, 1 \mathrm{H}), 8.28(\mathrm{dd}, J=1.7,7.7 \mathrm{~Hz}, 1 \mathrm{H}) ;{ }^{13} \mathrm{C}-\mathrm{NMR}\left(\mathrm{DMSO}-d_{6}\right) \delta 32.6,41.5$, 45.2, 45.6, 55.4, 66.0 (2C), 111.5, 119.2, 120.6, 124.3, 126.5, 128.8, 141.6, 155.3, 168.1; LC-MS (ESI) $\mathrm{m} / \mathrm{z}$ $317\left([\mathrm{M}+1]^{+}\right)$.

1-Morpholino-3-(4-p-tolyl-1H-1,2,3-triazol-1-yl)propan-1-one (2aci). Colorless oil; Yield: 93\%. ${ }^{1} \mathrm{H}-\mathrm{NMR}$ $\left(\mathrm{CDCl}_{3}\right) \delta 2.35(\mathrm{~s}, 3 \mathrm{H}), 2.96(\mathrm{t}, J=6.2 \mathrm{~Hz}, 2 \mathrm{H}), 3.37(\mathrm{t}, J=4.9 \mathrm{~Hz}, 2 \mathrm{H}), 3.55-3.61(\mathrm{~m}, 6 \mathrm{H}), 4.72(\mathrm{t}$, $J=6.2 \mathrm{~Hz}, 2 \mathrm{H}), 7.20(\mathrm{~d}, J=7.9 \mathrm{~Hz}, 2 \mathrm{H}), 7.68(\mathrm{~d}, J=8.1 \mathrm{~Hz}, 2 \mathrm{H}), 7.87(\mathrm{~s}, 1 \mathrm{H}) ;{ }^{13} \mathrm{C}-\mathrm{NMR}\left(\mathrm{DMSO}-d_{6}\right) \delta$ 20.8, 32.4, 41.5, 45.2, 45.8, 65.96, 65.98, 121.4, 125.0, 128.1, 129.4, 137.0, 146.1, 168.0; LC-MS (ESI) $\mathrm{m} / \mathrm{z}$ $301\left([\mathrm{M}+1]^{+}\right)$.

1-Morpholino-3-(4-m-tolyl-1H-1,2,3-triazol-1-yl)propan-1-one (2acj). Colorless oil; Yield: $98 \% .{ }^{1} \mathrm{H}-\mathrm{NMR}$ $\left(\mathrm{CDCl}_{3}\right) \delta 2.37(\mathrm{~s}, 3 \mathrm{H}), 2.96(\mathrm{t}, J=6.2 \mathrm{~Hz}, 2 \mathrm{H}), 3.38(\mathrm{t}, J=4.9 \mathrm{~Hz}, 2 \mathrm{H}), 3.56-3.62(\mathrm{~m}, 6 \mathrm{H}), 4.74(\mathrm{t}$, 
$J=6.2 \mathrm{~Hz}, 2 \mathrm{H}), 7.12(\mathrm{~d}, J=7.8 \mathrm{~Hz}, 1 \mathrm{H}), 7.28(\mathrm{t}, J=7.7 \mathrm{~Hz}, 1 \mathrm{H}), 7.57(\mathrm{~d}, J=7.8 \mathrm{~Hz}, 1 \mathrm{H}), 7.65(\mathrm{~s}, 1 \mathrm{H})$, $7.91(\mathrm{~s}, 1 \mathrm{H}) ;{ }^{13} \mathrm{C}-\mathrm{NMR}$ (DMSO- $\left.d_{6}\right) \delta 21.1,32.4,41.5,45.2,45.8,65.98,66.00,121.7,122.3,125.6,128.4$, 128.8, 130.7, 138.0, 146.2, 168.1; LC-MS (ESI) $m / z 301\left([\mathrm{M}+1]^{+}\right)$.

1-Morpholino-3-(4-o-tolyl-1H-1,2,3-triazol-1-yl)propan-1-one (2ack). Light yellow oil; Yield: 90\%. ${ }^{1} \mathrm{H}-\mathrm{NMR}\left(\mathrm{CDCl}_{3}\right) \delta 2.45(\mathrm{~s}, 3 \mathrm{H}), 3.00(\mathrm{t}, J=6.3 \mathrm{~Hz}, 2 \mathrm{H}), 3.40(\mathrm{t}, J=4.9 \mathrm{~Hz}, 2 \mathrm{H}), 3.57-3.62(\mathrm{~m}, 6 \mathrm{H})$, $4.77(\mathrm{t}, J=6.3 \mathrm{~Hz}, 2 \mathrm{H}), 7.21-7.27(\mathrm{~m}, 3 \mathrm{H}), 7.73(\mathrm{~m}, 1 \mathrm{H}), 7.82(\mathrm{~s}, 1 \mathrm{H}) ;{ }^{13} \mathrm{C}-\mathrm{NMR}$ (DMSO- $\left.d_{6}\right) \delta 21.1,32.5$, $41.5,45.2,45.8,66.0,66.02,123.6,126.0,127.7,128.1,130.1,130.9,134.8,145.4,168.1$; LC-MS (ESI) $\mathrm{m} / \mathrm{z}$ $301\left([\mathrm{M}+1]^{+}\right)$.

3-\{4-[4-(Dimethylamino)phenyl]-1H-1,2,3-triazol-1-yl\}-1-morpholinopropan-1-one (2acl). Light yellow solid; Yield: 99\%. ${ }^{1} \mathrm{H}-\mathrm{NMR}\left(\mathrm{CDCl}_{3}\right) \delta 2.96(\mathrm{t}, J=6.3 \mathrm{~Hz}, 2 \mathrm{H}), 2.97(\mathrm{~s}, 6 \mathrm{H}), 3.38(\mathrm{t}, J=4.9 \mathrm{~Hz}, 2 \mathrm{H})$, $3.57-3.62(\mathrm{~m}, 6 \mathrm{H}), 4.72(\mathrm{t}, J=6.3 \mathrm{~Hz}, 2 \mathrm{H}), 6.75(\mathrm{~d}, J=9.0 \mathrm{~Hz}, 2 \mathrm{H}), 7.67(\mathrm{~d}, J=9.0 \mathrm{~Hz}, 2 \mathrm{H}), 7.77(\mathrm{~s}, 1 \mathrm{H})$; ${ }^{13}$ C-NMR (DMSO- $\left.d_{6}\right) \delta 32.5,40.0,41.5,45.2,45.7,65.97,65.99,112.4,119.9,126.0,130.2,146.7,149.9$, 168.1; LC-MS (ESI) $m / z 330\left([\mathrm{M}+1]^{+}\right)$.

1-Morpholino-3-[4-(4-phenoxyphenyl)-1H-1,2,3-triazol-1-yl]propan-1-one (2acm). Light yellow oil; Yield: 99\%. ${ }^{1} \mathrm{H}-\mathrm{NMR}\left(\mathrm{CDCl}_{3}\right) \delta 2.98(\mathrm{t}, J=6.1 \mathrm{~Hz}, 2 \mathrm{H}), 3.39(\mathrm{t}, J=4.8 \mathrm{~Hz}, 2 \mathrm{H}), 3.58-3.63(\mathrm{~m}, 6 \mathrm{H}), 4.75(\mathrm{t}$, $J=6.1 \mathrm{~Hz}, 2 \mathrm{H}), 7.02-7.05(\mathrm{~m}, 4 \mathrm{H}), 7.12(\mathrm{~m}, 1 \mathrm{H}), 7.32-7.35(\mathrm{~m}, 2 \mathrm{H}), 7.77(\mathrm{~d}, J=8.7 \mathrm{~Hz}, 2 \mathrm{H}), 7.89(\mathrm{~s}, 1 \mathrm{H})$; ${ }^{13}$ C-NMR (DMSO- $d_{6}$ ) $\delta 32.44,41.5,45.2,45.8,65.96,65.98,118.8,118.9,123.6,124.2,126.2,126.8,130.1$, 145.6, 156.3, 156.4, 168.0; LC-MS (ESI) $m / z 379\left([\mathrm{M}+1]^{+}\right)$.

3-[4-(2,4-Difluorophenyl)-1H-1,2,3-triazol-1-yl]-1-morpholinopropan-1-one (2acn). Light yellow solid; Yield: 92. ${ }^{1} \mathrm{H}-\mathrm{NMR}\left(\mathrm{CDCl}_{3}\right) \delta 3.02(\mathrm{t}, J=6.3 \mathrm{~Hz}, 2 \mathrm{H}), 3.41(\mathrm{t}, J=4.9 \mathrm{~Hz}, 2 \mathrm{H}), 3.60-3.65(\mathrm{~m}, 6 \mathrm{H})$, $4.79(\mathrm{t}, J=6.3 \mathrm{~Hz}, 2 \mathrm{H}), 6.90(\mathrm{ddd}, J=2.4,8.7,11.0 \mathrm{~Hz}, 1 \mathrm{H}), 6.99(\mathrm{~m}, 1 \mathrm{H}), 8.03(\mathrm{~d}, J=3.8 \mathrm{~Hz}$, $1 \mathrm{H}), 8.24(\mathrm{dt}, J=6.5,8.6 \mathrm{~Hz}, 1 \mathrm{H}) ;{ }^{13} \mathrm{C}-\mathrm{NMR}\left(\mathrm{DMSO}_{6}\right) \delta 32.5,41.5,45.2,45.9,66.0(2 \mathrm{C}), 104.5(\mathrm{t}$, $\left.J_{\mathrm{CF}}=26.0 \mathrm{~Hz}\right), 112.3\left(\mathrm{dd}, J_{\mathrm{CF}}=21.1,3.7 \mathrm{~Hz}\right), 115.3\left(\mathrm{dd}, J_{\mathrm{CF}}=11.8,5.4 \mathrm{~Hz}\right), 123.9\left(\mathrm{~d}, J_{\mathrm{CF}}=10.4\right.$ $\mathrm{Hz}), 128.5\left(\mathrm{dd}, J_{\mathrm{CF}}=9.6,5.5 \mathrm{~Hz}\right), 138.8\left(\mathrm{~d}, J_{\mathrm{CF}}=2.5 \mathrm{~Hz}\right), 158.4\left(\mathrm{dd}, J_{\mathrm{CF}}=250.1,12.6 \mathrm{~Hz}\right), 161.7(\mathrm{dd}$, $\left.J_{\mathrm{CF}}=247.5,12.6 \mathrm{~Hz}\right), 168.1 ;$ LC-MS (ESI) $\mathrm{m} / \mathrm{z} 323\left([\mathrm{M}+1]^{+}\right)$.

3-(4-Benzyl-1H-1,2,3-triazol-1-yl)-1-morpholinopropan-1-one (2aco). Colorless oil; Yield: 98\%. ${ }^{1} \mathrm{H}-\mathrm{NMR}$ $\left(\mathrm{CDCl}_{3}\right) \delta 2.92(\mathrm{t}, J=6.4 \mathrm{~Hz}, 2 \mathrm{H}), 3.35(\mathrm{t}, J=4.9 \mathrm{~Hz}, 2 \mathrm{H}), 3.53-3.59(\mathrm{~m}, 6 \mathrm{H}), 4.04(\mathrm{~s}, 2 \mathrm{H}), 4.63(\mathrm{t}$, $J=6.4 \mathrm{~Hz}, 2 \mathrm{H}), 7.19(\mathrm{~m}, 1 \mathrm{H}), 7.21-7.29(\mathrm{~m}, 4 \mathrm{H}), 7.32(\mathrm{~s}, 1 \mathrm{H}) ;{ }^{13} \mathrm{C}-\mathrm{NMR}$ (DMSO- $\left.d_{6}\right) \delta 31.3,32.5,41.5$, $45.2,45.5,66.0$ (2C), 122.8, 126.1, 128.4, 128.5, 139.6, 145.8, 168.1; LC-MS (ESI) $m / z 301\left([\mathrm{M}+1]^{+}\right)$.

\section{Conclusions}

In summary, the yields for secondary $\alpha$-1,2,3-triazoloamides (80 examples) produced by solid-phase synthetic route ranged from 75 to $94 \%$ for six linear steps starting with Merrifield resin (the average yield for each step was over 95\%). The parallel solution-phase synthesis generated the target tertiary 1,2,3-triazoloamides (80 examples) with $97 \%-73 \%$ yields for three linear steps from the reaction of amines and chloro-acid chlorides. In addition, the target 1,2,3-triazoloamides were obtained in high purities (>95\%) as judged from LC-MS and ${ }^{1} \mathrm{H}-\mathrm{NMR}$ analyses. This investigation, has led to the development of the solid- and solution-phase route for the synthesis of various 1,2,3-triazoloamides that contain three diversity sites that were introduced in reactions involving amines $\left(R^{1}\right.$ and $\left.R^{2}\right)$, chloro-acid chlorides $(A)$, and terminal acetylenes $\left(R^{3}\right)$. The strategy allows for a ready access to a large library and is potentially applicable to the preparation of other 1,2,3-triazole derivatives.

Acknowledgments: This research was supported by the National Research Foundation of Korea (NRF) grants (NRF-2014R1A1A1037635 and NRF-2014R1A2A1A11052798) from the Basic Science Research Program, the Ministry of Science, ICT and Future Planning, Republic of Korea, and by a grant of the Korea Health Technology R\&D Project through the Korea Health Industry Development Institute (KHIDI), funded by the Ministry of Health \& Welfare, Republic of Korea (HI11C1300(A111345)). 
Author Contributions: T.L. and D.J.B. conceived and designed the experiments; D.L., D.K., S.L., T.K., J.K., S.K., S.L., and J.-S.B. performed experiments; K.-H.L., K.-S.S., C.-W.C., and Y.K.S. analyzed data; T.L., D.L., and D.J.B. wrote the paper. All authors read and approved the final manuscript.

Conflicts of Interest: The authors declare no conflict of interest.

\section{References}

1. Lowe, D.B. Drug discovery: Combichem all over again. Nat. Chem. 2014, 6, 851-852. [CrossRef] [PubMed]

2. Kim, J.; Kim, H.; Park, S.B. Privileged structures: Efficient chemical "navigators" toward unexplored biologically relavant chemical spaces. J. Am. Chem. Soc. 2014, 136, 14629-14638. [CrossRef] [PubMed]

3. Lee, T.; Gong, Y.-D. Solid-phase parallel synthesis of drug-like artificial $2 H$-benzopyran libraries. Molecules 2012, 17, 5467-5496. [CrossRef] [PubMed]

4. Dolle, R.E.; le Bourdonnec, B.; Worm, K.; Morales, G.A.; Thomas, C.J.; Zhang, W. Comprehensive survey of chemical libraries for drug discovery and chemical biology: 2009. J. Comb. Chem. 2010, 12, 765-806. [CrossRef] [PubMed]

5. Gong, Y.-D.; Lee, T. Combinatorial syntheses of five-membered ring heterocycles using carbon disulfide and a solid support. J. Comb. Chem. 2010, 12, 393-409. [CrossRef] [PubMed]

6. Kim, S.; Cho, M.; Lee, T.; Lee, S.; Min, H.-Y.; Lee, S.K. Design, synthesis, and preliminary biological evaluation of a novel triazole analogue of ceramide. Bioorg. Med. Chem. Lett. 2007, 17, 4584-4587. [CrossRef] [PubMed]

7. Lee, T.; Cho, M.; Ko, S.-Y.; Youn, H.-J.; Baek, D.J.; Cho, W.-J.; Kang, C.-Y.; Kim, S. Synthesis and evaluation of 1,2,3-triazole containing analogues of the immunostimulant $\alpha$-Galcer. J. Med. Chem. 2007, 50, 585-589. [CrossRef] [PubMed]

8. Wang, Q.; Chittaboina, S.; Barnhill, H.N. Advances in 1,3-dipolar cycloaddition reaction of azides and alkynes-a prototype of "Click" chemistry. Lett. Org. Chem. 2005, 2, 293-301. [CrossRef]

9. Bock, V.D.; Hiemstra, H.; van Maarseveen, J.H. Cu${ }^{\mathrm{I}}$-catalyzed alkyne-azide "Click" cycloaddtions from a mechanistic and synthetic perspective. Eur. J. Org. Chem. 2006, 2006, 51-68. [CrossRef]

10. Kolb, H.C.; Finn, M.G.; Sharpless, K.B. Click chemistry: Diverse chemical function from a few good reactions. Angew. Chem. Int. Ed. 2001, 40, 2004-2021. [CrossRef]

11. Stachel, S.J.; Sanders, J.M.; Henze, D.A.; Rudd, M.T.; Su, H.-P.; Li, Y.; Nanda, K.K.; Egbertson, M.S.; Manley, P.J.; Jones, K.L.G.; et al. Maximizing diversity from a kinase screen: Identification of novel and selective pan-Trk inhibitors for chronic pain. J. Med. Chem. 2014, 57, 5800-5816. [CrossRef] [PubMed]

12. Addla, D.; Jallapally, A.; Gurram, D.; Yogeeswari, P.; Sriram, D.; Kantevari, S. Design, synthesis and evaluation of 1,2,3-triazoleadamantylacetamide hybrids as potent inhibitors of Mycobacterium tuberculosis. Bioorg. Med. Chem. Lett. 2014, 24, 1974-1979. [CrossRef] [PubMed]

13. Mareddy, J.; Nallapati, S.B.; Anireddy, J.; Devi, Y.P.; Mangamoori, L.M.; Kapavarapu, L.; Pal, S. Synthesis and biological evaluation of nimesulide based new class of triazole derivatives as potential PDE4B inhibitors against cancer cells. Bioorg. Med. Chem. Lett. 2013, 23, 6721-6727.

14. Stacy, D.M.; le Quement, S.T.; Hansen, C.L.; Clausen, J.W.; Tolker-Nielsen, T.; Brummond, J.W.; Givskov, M.; Nielsen, T.E.; Blackwell, H.E. Synthesis and biological evaluation of triazole-containing $N$-acyl homoserine lactones as quorum sensing modulators. Org. Biomol. Chem. 2013, 11, 938-954. [CrossRef] [PubMed]

15. Joshi, M.C.; Wicht, K.J.; Taylor, D.; Hunter, R.; Smith, P.J.; Egan, T.J. In vitro antimalarial activity, $\beta$-haematin inhibition and structure-activity relationships in a series of quinoline triazoles. Eur. J. Med. Chem. 2013, 69, 338-347. [CrossRef] [PubMed]

16. Fischer, C.; Zultanski, S.L.; Zhou, H.; Methot, J.L.; Shah, S.; Nuthall, H.; Hughes, B.L.; Smotrov, N.; Hill, A.; Szewczak, A.A.; et al. Triazoloamides as potent $\gamma$-secretase modulators with reduced hERG liability. Bioorg. Med. Chem. Lett. 2012, 22, 3140-3146. [CrossRef] [PubMed]

17. He, Y.; Zeng, L.-F.; Yu, Z.-H.; He, R.; Liu, S.; Zhang, Z.-Y. Bicyclic benzofuran and indole-based salicylic acids as protein tyrosine phosphatase inhibitors. Bioorg. Med. Chem. 2012, 20, 1940-1946. [CrossRef] [PubMed]

18. Vang, T.; Xie, Y.; Liu, W.H.; Vidović, D.; Liu, Y.; Wu, S.; Smith, D.H.; Rinderspacher, A.; Chung, C.; Gong, G.; et al. Inhibition of lymphoid tyrosine phosphatase by benzofuran salicylic acids. J. Med. Chem. 2011, 54, 562-571. [CrossRef] [PubMed] 
19. Liu, Z.; Zhu, Q.; Li, F.; Zhang, L.; Leng, Y.; Zhang, A. N-(5-substituted thiazol-2-yl)-2-aryl-3-(tetrahydro-2Hpyran-4-yl)propanamides as glucokinase activators. Med. Chem. Commun. 2011, 2, 531-535. [CrossRef]

20. Gong, Y.-D.; Min, K.H.; Lee, T. An Efficient solid-phase synthesis of $\alpha-1,2,3$-triazoloamide derivatives via click chemistry. Bull. Korean Chem. Soc. 2011, 32, 2453-2456. [CrossRef]

21. Fivush, A.M.; Wilson, T.M. AMEBA: An acid sensitive aldehyde resin for solid phase synthesis. Tetrahedron Lett. 1997, 38, 7151-7154. [CrossRef]

22. Quan, Z.-J.; Xia, H.-D.; Zhang, Z.; Da, Y.-X.; Wang, X.-C. An efficient copper-catalyzed N-arylation of amides: synthesis of $N$-arylacrylamides and 4-amido- $N$-phenylbenzamides. Tetrahedron 2013, 69, 8368-8374. [CrossRef]

23. Zhang, Y.; Lv, Z.; Zhong, H.; Zhang, M.; Zhang, T.; Zhang, W.; Li, K. Efficient Heck cross-coupling of 3-iodo-benzopyrones with olefins under microwave irradiation without phosphine. Tetrahedron 2012, 68, 9777-9787. [CrossRef]

24. Silvestri, I.P.; Andemarian, F.; Khairallah, G.N.; Yap, S.W.; Quach, T.; Tsegay, S.; Williams, C.M.; O'Hair, R.A.J.; Donnelly, P.S.; Williams, S.J. Copper(I)-catalyzed cycloaddition of silver acetylides and azides: Incorporation of volatile acetylenes into the triazole core. Org. Biomol. Chem. 2011, 9, 6082-6088. [CrossRef] [PubMed]

25. Duval, R.; Kolb, S.; Braud, E.; Genest, D.; Garbay, C. Rapid discovry of triazolobenzylidene-thiazolopyrimidines (TBTP) as CDC25 Phosphatase inhibitors by parallel click chemistry and in situ screening. J. Comb. Chem. 2009, 11, 947-950. [CrossRef] [PubMed]

26. Bonnet, D.; Riché, S.; Loison, S.; Dagher, R.; Frantz, M.-C.; Boudier, L.; Rahmeh, R.; Mouillac, B.; Haiech, J.; Hibert, M. Solid-Phase organic tagging resins for labeling biomolecules by 1,3-dipolar cycloaddition: Application to the synthesis of a fluorescent non-peptidic vasopressin receptor ligand. Chem. Eur. J. 2008, 14, 6247-6254. [CrossRef] [PubMed]

27. Donnelly, P.S.; Zanatta, S.D.; Zammit, S.C.; White, J.M.; Williams, S.J. “Click” cycloaddition catalysts: Copper(I) and copper(II) tris(triazolylmethyl)amine complexes. Chem. Commun. 2008, 7, 2459-2461. [CrossRef] [PubMed]

28. Lv, G.; Mai, W.; Jin, R.; Gao, L. Immobilization of dipyridyl complex to magnetic nanoparticle via click chemistry as a recyclable catalyst for suzuki cross-coupling reactions. Synlett 2008, 1418-1422. [CrossRef]

29. Cao, J.; Huang, X. Solid-phase syntheis of heterocyclic nucleoside analogures: Substituted uracils tethered to isoxazoles, isoxazolines, and triazoles from a selenopolystyrene resin. J. Comb. Chem. 2008, 10, 526-533. [CrossRef] [PubMed]

30. Ananthanarayanan, C.; Ramakrishnan, V.T. Synthesis of 1,2,3-triazoles. Indian J. Chem. B 1989, 28B, $228-230$.

31. Kim, D.; Baek, D.J.; Lee, D.; Liu, K.-H.; Bae, J.-S.; Gong, Y.-D.; Min, K.H.; Lee, T. Efficient solid-phase synthesis of 2,4-disubstituted 5-carbamoyl-thiazole derivatives using a traceless support. Tetrahedron 2015, 71, 3367-3377. [CrossRef] [PubMed]

32. Sirakanyan, S.N.; Spinelli, D.; Geronikaki, A.; Hovakimyan, A.A.; Noravyan, A.S. On the reaction of 2-[(4-cyano-5,6,7,8-tetrahydroisoquinolin-3-yl)oxy]acetamides with bases: 1-amino-6,7,8,9-tetrahydrofuro[2,3-c] isoquinoline-2-carboxamides and 3-amino-4-cyano-5,6,7,8-tetrahydroisoquinolines via a Smiles-type rearrangement. Tetrahedron 2015, 71, 3263-3272. [CrossRef]

33. Lasalle, M.; Picon, S.; Boulahjar, R.; Hoguet, V.; van Obbergen, J.; Roussel, P.; Deprez, B.; Charton, J. Access to newly functionalized imidazole derivatives: Efficient synthesis of novel 5-amino-2-thioimidazoles using propylphosphonic anhydride ( $\left.{ }^{\circledR} \mathrm{T} 3 \mathrm{P}\right)$. Tetrahedron Lett. 2015, 56, 1011-1014. [CrossRef]

34. Brullo, C.; Massa, M.; Rocca, M.; Rotolo, C.; Guariento, S.; Rivera, D.; Ricciarelli, R.; Fedele, E.; Fossa, P.; Bruno, O. Synthesis, biological evaluation, and molecular modeling of new 3-(cyclopentyloxy)-4-methoxybenzaldehyde O-(2-(2,6-dimethylmorpholino)-2-oxoethyl) oxime (GEBR-7b) related phosphodiesterase 4D (PDE4D) inhibitors. J. Med. Chem. 2014, 57, 7061-7072. [CrossRef] [PubMed]

35. Ji, Q.; Yang, D.; Wang, X.; Chen, C.; Deng, Q.; Ge, Z.; Yuan, L.; Yang, X.; Liao, F. Design, synthesis and evaluation of novel quinazoline-2,4-dione derivatives as chitin synthase inhibitors and antifungal agents. Bioorg. Med. Chem. 2014, 22, 3405-3413. [CrossRef] [PubMed]

36. Molander, G.A.; Traister, K.M.; Barcellos, T. Palladium-catalyzed $\alpha$-arylation of 2-chloroacetates and 2-chloroacetamides. J. Org. Chem. 2013, 78, 4123-4131. [CrossRef] [PubMed] 
37. Youngsaye, W.; Dockendorff, C.; Vincent, B.; Hartland, C.L.; Bittker, J.A.; Dandapani, S.; Palmer, M.; Whitesell, L.; Lindquist, S.; Schreiber, S.L.; et al. Overcoming fluconazole resistance in Candida albicans clinical isolates with tetracyclic indoles. Bioorg. Med. Chem. Lett. 2012, 22, 3362-3365. [CrossRef] [PubMed]

38. Li, Z.-S.; Wang, W.-X.; Yang, J.-D.; Wu, Y.-W.; Zhang, W. Photoinduced and N-bromosuccinimide-mediated cyclization of 2-azido-N-phenylacetamides. Org. Lett. 2013, 15, 3820-3823. [CrossRef] [PubMed]

39. Hemasri, Y.; Srinivas, A. Regioselective synthesis of novel 1,4 disubstituted bis 1,2,3 triazoles: Click chemistry approach. Heterocycl. Lett. 2012, 2, 79-83.

40. Reed, C.S.; Huigens, R.W., III; Rogers, S.A.; Melander, C. Modulating the development of E. coli biofilms with 2-aminoimidazoles. Bioorg. Med. Chem. Lett. 2010, 20, 6310-6312. [CrossRef] [PubMed]

41. Huigens, R.W., III; Reyes, S.; Reed, C.S.; Bunders, C.; Rogers, S.A.; Steinhauer, A.T.; Melander, C. The chemical synthesis and antibiotic activity of a diverse library of 2-aminobenzimidazole small molecules against MRSA and multidrug-resistant A. baumannii. Bioorg. Med. Chem. 2010, 18, 663-674. [CrossRef] [PubMed]

Sample Availability: Samples of the compounds are not available.

(C) 2015 by the authors; licensee MDPI, Basel, Switzerland. This article is an open access article distributed under the terms and conditions of the Creative Commons by Attribution (CC-BY) license (http://creativecommons.org/licenses/by/4.0/). 